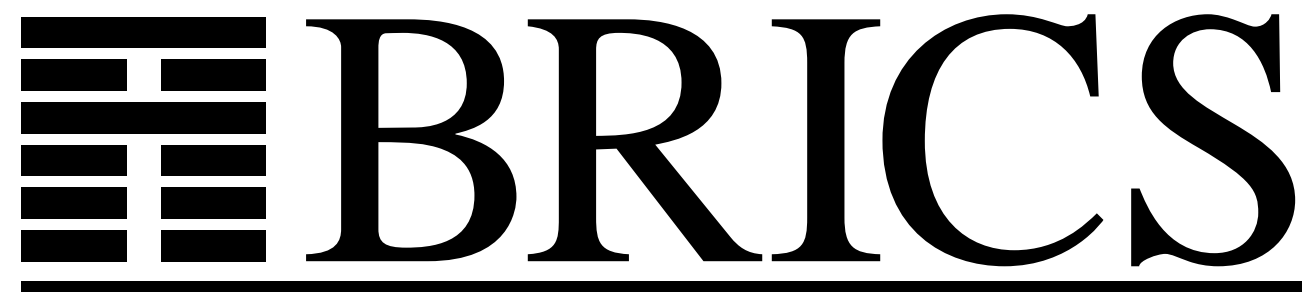

Basic Research in Computer Science

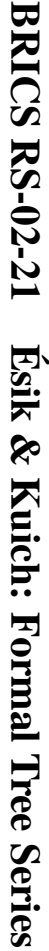

\title{
Formal Tree Series
}

Zoltán Ésik

Werner Kuich 
Copyright (c) 2002, Zoltán Ésik \& Werner Kuich.

BRICS, Department of Computer Science

University of Aarhus. All rights reserved.

Reproduction of all or part of this work is permitted for educational or research use on condition that this copyright notice is included in any copy.

See back inner page for a list of recent BRICS Report Series publications. Copies may be obtained by contacting:

\author{
BRICS \\ Department of Computer Science \\ University of Aarhus \\ Ny Munkegade, building 540 \\ DK-8000 Aarhus C \\ Denmark \\ Telephone: +45 89423360 \\ Telefax: $\quad+4589423255$ \\ Internet: BRICS@brics.dk
}

BRICS publications are in general accessible through the World Wide Web and anonymous FTP through these URLs:

http://www.brics.dk

ftp: / / ftp.brics.dk

This document in subdirectory RS / 02/21/ 


\title{
Formal tree series
}

\author{
Zoltán Ésik* \\ Institute of Informatics \\ University of Szeged \\ 6720 Szeged, Árpád tér 2 \\ esik@inf.u-szeged.hu
}

\author{
Werner Kuich* \\ Abteilung für Theoretische Informatik \\ Institut für Algebra und Computermathematik \\ Technische Universität Wien \\ Wiedner Hauptstraße 8-10, A-1040 Wien \\ kuich@tuwien.ac.at
}

\begin{abstract}
In this survey we generalize some results on formal tree languages, tree grammars and tree automata by an algebraic treatment using semirings, fixed point theory, formal tree series and matrices. The use of these mathematical constructs makes definitions, constructions, and proofs more satisfactory from an mathematical point of view than the customary ones. The contents of this survey paper is indicated by the titles of the sections:

1. Introduction

2. Preliminaries

3. Tree automata and systems of equations

4. Closure properties and a Kleene Theorem for recognizable tree series

5. Pushdown tree automata, algebraic tree systems, and a Kleene Theorem

6. Tree series transducers

7. Full abstract families of tree series

8. Connections to formal power series
\end{abstract}

*Partially supported by the "Stiftung Aktion Österreich-Ungarn". During the preparation of this paper the first author was supported by BRICS and the University of Aalborg. 


\section{Introduction}

The purpose of this survey paper is to generalize some results on formal tree languages, tree grammars and tree automata by an algebraic treatment using semirings, fixed point theory, formal tree series and matrices. The use of these mathematical constructs yields the following advantages:

(i) The constructions needed in the proofs of the results are mainly the usual ones.

(ii) The descriptions of the constructions by formal tree series and matrices are more precise than the customary ones.

(iii) The proofs are separated from the constructions and are more satisfactory from a mathematical point of view. Often they are shorter than the usual proofs.

(iv) The results are more general than the usual ones. Depending on the semiring used, the results are valid for classical tree automata or tree grammars, classical tree automata or tree grammars with ambiguity considerations, probabilistic tree automata or tree grammars, etc.

The prize to pay for these advantages is a knowledge of the basics of semiring theory and fixed point theory.

It is assumed that the reader has some basic knowledge of semirings (see e.g., Kuich [36]), fixed point theory (see Bloom, Ésik [6]), and tree languages and tree automata (see Gécseg, Steinby [24, 25], Comon, Dauchet, Gilleron, Jaquemard, Lugiez, Tison, Tommasi [14]). Formal tree series were introduced by Berstel, Reutenauer [5], and then extensively studied by Bozapalidis [7, 8, 9, 10, 11], Bozapalidis, Rahonis [12], Kuich [38, 39, 40, 41, 43], Engelfriet, Fülöp, Vogler [19] and Fülöp, Vogler [23].

We now give a short description of the contents of this survey paper.

In Section 2, we define those algebraic structures that will be used throughout the paper. These structures include complete and continuous monoids, semirings, and distributive $\Sigma$-algebras, where $\Sigma$ is any signature. We introduce tree series and characterize the distributive $\Sigma$-algebras of tree series (with coefficients in a continuous semiring) by a universal property. We use this characterization to derive properties of tree series substitutions. Then we review the basics of the fixed point theory of continuous functions, including the theorem of Bekić, De Bakker and Scott regarding the solution of simultaneous least fixed point equations. In Section 3 we define tree automata and systems of equations whose right sides consist of tree series. These notions are a framework for the considerations of finite tree automata and pushdown tree automata, and polynomial systems. The main result of this section is that (finite, polynomial) tree automata and (finite, polynomial) systems are equivalent mechanisms. In Section 4 we prove a Kleene Theorem for recognizable tree series that allows 
also the definition of recognizable tree series by expressions which are analogous to regular expressions. In Section 5, pushdown tree automata and algebraic tree systems are introduced and shown that these mechanisms are equivalent. Moreover, a Kleene Theorem for algebraic tree series is proved. Top-down tree series transducers are introduced in Section 6. We concentrate on (top-down) linear nondeleting recognizable tree series transducers and prove that they preserve recognizability of tree series. Full abstract families of tree series (briefly, full AFTs) are families of tree series closed under linear nondeleting recognizable tree transductions and certain specific "rational" operations. These full AFTs are introduced in Section 7. It is shown that the families of recognizable tree series and of algebraic tree series are full AFTs. The last section brings connections of formal tree series to formal power series. We first show that the macro power series (a generalization of the indexed languages) are the yield of algebraic tree series. Moreover, we prove a Kleene Theorem for macro power series (and indexed languages). Then we show that algebraic power series are the yield of recognizable tree series. Finally, we prove the important result that the yield of a full AFT is a full abstract family of power series.

\section{Preliminaries}

In this section we first consider commutative monoids. The definitions and results on commutative monoids are mainly due sometimes in the framework of semiring theory - to Eilenberg [17], Goldstern [28], Karner [33], Krob [34], Kuich [35, 36], Kuich, Salomaa [45], Manes, Arbib [47], Sakarovitch [53]. Our notion of continuous monoid is a specialization of the continuous algebras as defined, e. g., in Guessarian [31], Goguen, Thatcher, Wagner, Wright [27], Adamek, Nelson, Reiterman [2].

In the second part of this section we consider distributive algebras. The definitions and results on distributive algebras are heavily influenced by Bozapalidis [10], especially by his notion of a $K$ - $\Gamma$-algebra. He noticed that the multilinear mappings of his well $\omega$-additive $K$ - $\Gamma$-algebras assure that certain important mappings induced by formal power series are continuous. (See Theorem 2 of Bozapalidis [10].) We have tried in the forthcoming definition of a distributive algebra to simplify the used type of algebra but to save the important results. Semirings are then introduced as a very important distributive algebra.

In the third part of this section we introduce formal tree series. These formal tree series form a distributive algebra.

In our paper we often will need certain results of fixed point theory. Hence, in the fourth part of this section, we give a short introduction into the fixed point theory of continuous functions and refer to a few results of this theory.

In the final part of this section we consider some important mappings connected with formal tree series and show that they are continuous. 
A commutative monoid $\langle A,+, 0\rangle$ is called ordered iff it is equipped with a partial order $\leq$ preserved by the + operation such that $0 \leq a$ holds for all $a \in A$. It then follows that $a \leq a+b$, for all $a, b \in A$. In particular, a commutative monoid $\langle A,+, 0\rangle$ is called naturally ordered iff the relation $\sqsubseteq$ defined by: $a \sqsubseteq b$ iff there exists a $c$ such that $a+c=b$, is a partial order. Morphisms of ordered monoids preserve the order.

A commutative monoid $\langle A,+, 0\rangle$ is called complete iff it has sums for all families $\left(a_{i} \mid i \in I\right)$ of elements of $A$, where $I$ is an arbitrary index set, such that the following conditions are satisfied:

(i) $\sum_{i \in \emptyset} a_{i}=0, \sum_{i \in\{j\}} a_{i}=a_{j}, \sum_{i \in\{j, k\}} a_{i}=a_{j}+a_{k}$, for $j \neq k$,

(ii) $\sum_{j \in J}\left(\sum_{i \in I_{j}} a_{i}\right)=\sum_{i \in I} a_{i}$, if $\bigcup_{j \in J} I_{j}=I$ and $I_{j} \cap I_{j^{\prime}}=\emptyset$ for $j \neq j^{\prime}$.

A morphism of complete monoids preserves all sums.

Recall that a nonempty subset $D$ of a partially ordered set $P$ is called directed iff each pair of elements of $D$ has an upper bound in $D$. Moreover, a function $f: P \rightarrow Q$ between partially orderet sets is continuous iff it preserves the least upper bound of directed sets, i.e., when $f(\sup D)=\sup f(D)$, for all directed sets $D \subseteq P$ such that $\sup D$ exists. It follows that any continuous function preserves the order.

An ordered commutative monoid $\langle A,+, 0\rangle$ is called a continuous monoid iff each directed subset of $A$ has a least upper bound and the + operation preserves the least upper bound of directed sets, i.e., when

$$
a+\sup D=\sup (a+D),
$$

for all directed sets $D \subseteq A$ and for all $a \in A$. Here, $a+D$ is the set $\{a+x \mid x \in$ $D\}$. A morphism of continuous monoids is a continuous monoid homomorphism.

It is known that an ordered commutative monoid $A$ is continuous iff each chain in $A$ has a least upper bound and the + operation preserves least upper bounds of chains, i. e., when $a+\sup C=\sup a+C$ holds for all nonempty chains $C$ in $A$. (See Markowsky [48].)

Proposition 2.1 Any continuous monoid $\langle A,+, 0\rangle$ is a complete monoid equipped with the following sum operation:

$$
\sum_{i \in I} a_{i}=\sup \left\{\sum_{i \in E} a_{i} \mid E \subseteq I, E \text { finite }\right\}
$$

for all index sets $I$ and all families $\left(a_{i} \mid i \in I\right)$ in $A$. Any morphism between continuous monoids is a complete monoid morphism.

A signature is a nonempty set $\Sigma$, whose elements are called operation symbols, together with a mapping ar $: \Sigma \rightarrow \mathbb{N}$, called the arity function, assigning to each operation symbol its finite arity ( $\mathbb{N}$ denotes the nonnegative integers). 
We write $\Sigma=\Sigma_{0} \cup \Sigma_{1} \cup \ldots \cup \Sigma_{k} \cup \ldots$, where $\Sigma_{k}, k \geq 0$, contains the operator symbols of arity $k$.

Let $\Sigma$ be a signature. Recall that a $\Sigma$-algebra $\langle A, \Omega\rangle$ consists of a nonempty set $A$ and a family of operations $\Omega=\Omega_{0} \cup \Omega_{1} \cup \ldots \cup \Omega_{k} \cup \ldots$ on $A$. Here $\Omega_{k}=\left\{\omega_{\sigma} \mid \sigma \in \Sigma_{k}\right\}$, and $\omega_{\sigma}: A^{k} \rightarrow A$ is a $k$-ary operation for each $\sigma \in$ $\Sigma_{k}, k \geq 0$. (See Gécseg, Steinby [24], Grätzer [29], Lausch, Nöbauer [46], Wechler [59].) Usually, we denote $\sigma \in \Sigma$ and $\omega_{\sigma} \in \Omega$ by the same letter. The algebra $\langle A,+, 0, \Omega\rangle$, where $\langle A,+, 0\rangle$ is a commutative monoid and $\langle A, \Omega\rangle$ is a $\Sigma$-algebra, is called a distributive $\Sigma$-algebra iff the following two conditions are satisfied for all $\omega \in \Omega_{k}$ and all $a, a_{1}, \ldots, a_{k} \in A, k \geq 1$ :

(i) $\omega\left(a_{1}, \ldots, a_{j-1}, 0, a_{j+1}, \ldots, a_{k}\right)=0$ for all $1 \leq j \leq k$,

(ii) $\omega\left(a_{1}, \ldots, a_{j-1}, a_{j}+a, a_{j+1}, \ldots, a_{k}\right)=$ $\omega\left(a_{1}, \ldots, a_{j-1}, a_{j}, a_{j+1}, \ldots, a_{k}\right)+\omega\left(a_{1}, \ldots, a_{j-1}, a, a_{j+1}, \ldots, a_{k}\right)$ for all $1 \leq j \leq k$.

A morphism of distributive $\Sigma$-algebras preserves both the monoid structure and the operations $\omega$. In the sequel, $\Sigma=\Sigma_{0} \cup \Sigma_{1} \cup \ldots \cup \Sigma_{k} \cup \ldots$ will always denote a signature. In connection with trees, a signature will be called ranked alphabet, where the rank of an operation symbol is its arity.

A distributive $\Sigma$-algebra $\langle A,+, 0, \Omega\rangle$ is briefly denoted by $A$ if,+ 0 and $\Omega$ are understood. Similar algebras are considered in Courcelle [15] and Bozapalidis $[10]$.

A distributive $\Sigma$-algebra $\langle A,+, 0, \Omega\rangle$ is termed ordered iff $\langle A,+, 0\rangle$ is ordered and if each operation $\omega \in \Omega$ preserves the order in each argument. When the order is the natural order, this latter condition holds by distributivity. A morphism of ordered distributive $\Sigma$-algebras is an order preserving distributive $\Sigma$-algebra morphism.

A distributive $\Sigma$-algebra $\langle A,+, 0, \Omega\rangle$ is called complete iff $\langle A,+, 0\rangle$ is complete and the following additional condition is satisfied for all $\omega \in \Omega_{k}$, index sets $I_{1}, \ldots, I_{k}$, and $a_{i_{1}}, \ldots, a_{i_{k}} \in A, i_{1} \in I_{1}, \ldots, i_{k} \in I_{k}, k \geq 1$ :

$$
\omega\left(\sum_{i_{1} \in I_{1}} a_{i_{1}}, \ldots, \sum_{i_{k} \in I_{k}} a_{i_{k}}\right)=\sum_{i_{1} \in I_{1}} \ldots \sum_{i_{k} \in I_{k}} \omega\left(a_{i_{1}}, \ldots, a_{i_{k}}\right) .
$$

Eventually, an ordered distributive $\Sigma$-algebra $\langle A,+, 0, \Omega\rangle$ is called continuous iff $\langle A,+, 0\rangle$ is continuous and if the operations $\omega \in \Omega_{k}$ are continuous: For each $a_{1}, \ldots, a_{i-1}, a_{i+1}, \ldots, a_{k} \in A, 1 \leq i \leq k$, and for each directed set $D \subseteq A$,

$$
\omega\left(a_{1}, \ldots, \sup D, \ldots, a_{k}\right)=\sup \omega\left(a_{1}, \ldots, D, \ldots, a_{k}\right) .
$$

A morphism of complete (resp. continuous) distributive $\Sigma$-algebras is both a complete (resp. continuous) monoid morphism and a distributive $\Sigma$-algebra morphism. From Proposition 2.1 we easily derive: 
Proposition 2.2 Any continuous distributive $\Sigma$-algebra is complete. Any morphism of continuous distributive $\Sigma$-algebras is a morphism of complete distributive $\Sigma$-algebras.

Let $\langle A,+, 0\rangle$ be a commutative monoid, and $\cdot$ be a binary and 1 a nullary operation on $A$ such that $\langle A, \cdot, 1\rangle$ is a monoid. Then the distributive $\Sigma$-algebra $\langle A,+, 0,(\cdot, 1)\rangle$, written $\langle A,+, \cdot, 0,1\rangle$, is called semiring. A semiring $\langle A,+, \cdot, 0,1\rangle$ is called commutative iff $\langle A, \cdot, 1\rangle$ is a commutative monoid. It is called (naturally) ordered, complete or continuous iff it is (naturally) ordered, complete or continuous, respectively, as a distributive $(\cdot, 1)$-algebra. Morphisms of ordered semirings preserve the partial order. (Again, this condition holds automatically when the partial order is the natural order.) Morphisms of complete or continuous semirings are complete, or continuous distributive $(\cdot, 1)$-algebra morphisms.

Example 1.1. Let $\Sigma=\bigcup_{k \geq 0} \Sigma_{k}, \Sigma_{k}=\left\{\omega_{k}\right\}, k \geq 0$. Consider a semiring $\langle A,+, \cdot, 0,1\rangle$ and define $\omega_{k}, k \geq 0$, to be the following $k$-ary operations: the nullary constant $\omega_{0}$ is 1 , the unary operation $\omega_{1}$ is the identity mapping and the $k$-ary operation $\omega_{k}$ is the $k$-fold product, i. e., $\omega\left(a_{1}, \ldots, a_{k}\right)=a_{1} \cdots a_{k}, k \geq 2$. Then $\langle A,+, 0, \Omega\rangle, \Omega=\left(\omega_{k} \mid k \in \mathbb{N}\right)$ is a distributive $\Sigma$-algebra. If $\langle A,+, \cdot, 0,1\rangle$ is a continuous semiring then $\langle A,+, 0, \Omega\rangle$ is a continuous distributive $\Sigma$-algebra.

Example 1.2. Consider a semiring $\langle A,+, \cdot, 0,1\rangle$. Let $\Sigma=\Sigma_{0} \cup \Sigma_{1}, \Sigma_{0}=\{\omega\}$, $\Sigma_{1}=\left\{\omega_{a} \mid a \in A\right\}$. Then the semiring $A$ can be "simulated" by a distributive $\Sigma$-algebra $\langle A,+, 0, \Omega\rangle$, where $\Omega_{0}=\{\omega\}, \Omega_{1}=\left\{\omega_{a} \mid a \in A\right\}$ and $\Omega_{k}=\emptyset$ for $k \geq 2$. Here $\omega$ is the nullary constant 1 and, for all $a, b \in A, \omega_{a}(b)=a \cdot b$.

Additionally to the laws of a distributive $\Sigma$-algebra, the following laws are satisfied for all $a, a_{1}, a_{2}, b \in A$ :

$$
\begin{gathered}
\omega_{a_{1}}\left(\omega_{a_{2}}(b)\right)=\omega_{a_{1} \cdot a_{2}}(b), \quad \omega_{a_{1}+a_{2}}(b)=\omega_{a_{1}}(b)+\omega_{a_{2}}(b), \\
\omega_{0}(b)=0, \quad \omega_{1}(b)=b, \quad \omega_{a}(1)=a .
\end{gathered}
$$

In the sequel, $X$ will denote an alphabet of leaf symbols, disjoint from $\Sigma$. (Observe that an alphabet may be infinite.) By $T_{\Sigma}(X)$ we denote the set of trees formed over $\Sigma \cup X$. This set $T_{\Sigma}(X)$ is the smallest set formed according to the following conventions:

(i) if $\sigma \in \Sigma_{0} \cup X$ then $\sigma \in T_{\Sigma}(X)$,

(ii) if $\sigma \in \Sigma_{k}, k \geq 1$, and $t_{1}, \ldots, t_{k} \in T_{\Sigma}(X)$ then $\sigma\left(t_{1}, \ldots, t_{k}\right) \in T_{\Sigma}(X)$.

If $\Sigma_{0} \neq \emptyset$ then $X$ may be the empty set $(\emptyset$ denotes the empty set).

If $\Sigma$ is a finite ranked alphabet and $X$ is a finite alphabet of leaf symbols then $T_{\Sigma}(X)$ is generated by the context-free grammar $G=(\{S\}, \Sigma \cup X, P, S)$, where $P=\left\{S \rightarrow \omega(S, \ldots, S) \mid \omega \in \Sigma_{k}, k \geq 1\right\} \cup\left\{S \rightarrow \omega \mid \omega \in \Sigma_{0} \cup X\right\}$. 
Sometimes it is more suggestive to employ a pictorial representation: The tree $\omega \in \Sigma_{0} \cup X$ represents the rooted plane tree with just a single node labeled by $\omega$; the tree $\omega\left(t_{1}, \ldots, t_{k}\right), \omega \in \Sigma_{k}, t_{1}, \ldots, t_{k} \in T_{\Sigma}(X), k \geq 1$, represents the rooted plane tree where the root is labeled by $\omega$ and has sons $t_{1}, \ldots, t_{k}$ (in this order).

The set $T_{\Sigma}(X)$ may be turned into a $\Sigma$-algebra by defining, for each $\sigma \in \Sigma_{k}$ and $t_{1}, \ldots, t_{k} \in T_{\Sigma}(X), \omega_{\sigma}\left(t_{1}, \ldots, t_{k}\right)$ to be the tree $\sigma\left(t_{1}, \ldots, t_{k}\right)$. It is wellknown that equipped with these operations, $T_{\Sigma}(X)$ is freely generated by $X$ : Each function $h: X \rightarrow D$, where $D$ is a $\Sigma$-algebra, extends to a unique $\Sigma$ algebra morphism $T_{\Sigma}(X) \rightarrow D$.

We now turn to formal tree series. They will form a distributive $\Sigma$-algebra. Let $A$ be a semiring. Then we denote by $A\left\langle\left\langle T_{\Sigma}(X)\right\rangle\right\rangle$ the set of formal tree series over $T_{\Sigma}(X)$, i. e., the set of mappings $s: T_{\Sigma}(X) \rightarrow A$ written in the form $\sum_{t \in T_{\Sigma}(X)}(s, t) t$, where the coefficient $(s, t)$ is the value of $s$ for the tree $t \in T_{\Sigma}(X)$. For a formal tree series $s \in A\left\langle\left\langle T_{\Sigma}(X)\right\rangle\right\rangle$, we define the support of $s, \operatorname{supp}(s)=\left\{t \in T_{\Sigma}(X) \mid(s, t) \neq 0\right\}$. By $A\left\langle T_{\Sigma}(X)\right\rangle$ we denote the set of tree series in $A\left\langle\left\langle T_{\Sigma}(X)\right\rangle\right\rangle$ that have finite support. A power series with finite support is called polynomial.

We first define, for $s_{1}, s_{2} \in A\left\langle\left\langle T_{\Sigma}(X)\right\rangle\right\rangle$, the sum $s_{1}+s_{2} \in A\left\langle\left\langle T_{\Sigma}(X)\right\rangle\right\rangle$ by

$$
s_{1}+s_{2}=\sum_{t \in T_{\Sigma}(X)}\left(\left(s_{1}, t\right)+\left(s_{2}, t\right)\right) t .
$$

The zero tree series 0 is defined to be the tree series having all coefficients equal to 0. Clearly, $\left\langle A\left\langle\left\langle T_{\Sigma}(X)\right\rangle\right\rangle,+, 0\right\rangle$ is a commutative monoid. by

For $\omega \in \Sigma_{k}, k \geq 0$, we define the mapping $\bar{\omega}:\left(A\left\langle\left\langle T_{\Sigma}(X)\right\rangle\right\rangle\right)^{k} \rightarrow A\left\langle\left\langle T_{\Sigma}(X)\right\rangle\right\rangle$

$$
\bar{\omega}\left(s_{1}, \ldots, s_{k}\right)=\sum_{t_{1}, \ldots, t_{k} \in T_{\Sigma}(X)}\left(s_{1}, t_{1}\right) \ldots\left(s_{k}, t_{k}\right) \omega\left(t_{1}, \ldots, t_{k}\right),
$$

$s_{1}, \ldots, s_{k} \in A\left\langle\left\langle T_{\Sigma}(X)\right\rangle\right\rangle$.

Clearly, $\left\langle A\left\langle\left\langle T_{\Sigma}(X)\right\rangle\right\rangle,+, 0, \bar{\Sigma}\right\rangle$, where $\bar{\Sigma}=(\bar{\omega} \mid \omega \in \Sigma)$, is a distributive $\Sigma$ algebra, as is $\left\langle A\left\langle T_{\Sigma}(X)\right\rangle,+, 0, \bar{\Sigma}\right\rangle$ with the same operations. If $A$ is (naturally) ordered (resp. complete or continuous) then $A\left\langle\left\langle T_{\Sigma}(X)\right\rangle\right\rangle$ is again a (naturally) ordered (resp. complete or continuous) distributive $\Sigma$-algebra. The order on $A\left\langle\left\langle T_{\Sigma}(X)\right\rangle\right\rangle$ is the pointwise order. Also, when $A$ is ordered, $A\left\langle T_{\Sigma}(X)\right\rangle$ is an ordered distributive $\Sigma$-algebra.

Example 1.3. Formal tree series have the advantage that the coefficient of a tree in a series can be used to give information about some quantity connected with that tree.

(i) (See Example 2.1 of Berstel, Reutenauer [5].) Define the height $h(t)$ of a tree $t$ in $T_{\Sigma}(X)$ as follows:

$$
h(t)= \begin{cases}0 & \text { if } t \in \Sigma_{0} \cup X, \\ 1+\max \left\{h\left(t_{i}\right) \mid 1 \leq i \leq k\right\} & \text { if } t=\omega\left(t_{1}, \ldots, t_{k}\right), k \geq 1 .\end{cases}
$$


Now height is a formal tree series in $\mathbb{N}\left\langle\left\langle T_{\Sigma}(X)\right\rangle\right\rangle$ defined as

$$
\text { height }=\sum_{t \in T_{\Sigma}(X)} h(t) t .
$$

(ii) Consider formal tree series $s$ in $\mathbb{R}_{+}\left\langle\left\langle T_{\Sigma}(X)\right\rangle\right\rangle$ such that $0 \leq(s, t) \leq 1$ for all $t \in T_{\Sigma}(X)$. Then $(s, t)$ can be interpreted as a probability associated with the tree $t$. Here $\mathbb{R}_{+}$is the semiring of nonnegative reals.

(iii) Consider formal tree series $s$ in $\mathbb{N}^{\infty}\left\langle\left\langle T_{\Sigma}(X)\right\rangle\right\rangle$, where $\mathbb{N}^{\infty}=\mathbb{N} \cup\{\infty\}$. Then the coefficient $(s, t)$ of $t \in T_{\Sigma}(X)$ can be interpreted as the number (possibly $\infty$ ) of distinct generations of $t$ by some mechanism. (See Theorem 3.1.)

More examples can be found in Berstel, Reutenauer [5].

We now exhibit a universal property of the above constructions. Note that $A\left\langle\left\langle T_{\Sigma}(X)\right\rangle\right\rangle$ may be equipped with a scalar multiplication $A \times A\left\langle\left\langle T_{\Sigma}(X)\right\rangle\right\rangle \rightarrow$ $A\left\langle\left\langle T_{\Sigma}(X)\right\rangle\right\rangle,(a, s) \mapsto a s$, defined by $\langle a s, t)=a(s, t)$, for all $t \in T_{\Sigma}(X)$. When $s \in A\left\langle T_{\Sigma}(X)\right\rangle$, then also as $\in A\left\langle T_{\Sigma}(X)\right\rangle$. This operation satisfies the following equations:

$$
\begin{aligned}
a(b s) & =(a b) s \\
1 s & =s \\
(a+b) s & =a s+b s \\
a\left(s+s^{\prime}\right) & =a s+a s^{\prime} \\
a 0 & =0,
\end{aligned}
$$

for all $a, b \in A$ and $s, s^{\prime} \in A\left\langle\left\langle T_{\Sigma}(X)\right\rangle\right\rangle$. It follows that

$$
0 s=0,
$$

for all $s$. Moreover, when $A$ is commutative, we also have that

$$
\omega\left(a_{1} s_{1}, \ldots, a_{k} s_{k}\right)=a_{1} \ldots a_{k} \omega\left(s_{1}, \ldots, s_{k}\right),
$$

for all $\omega \in \Sigma_{k}, k \geq 0$, and for all $a_{i} \in A, s_{i} \in A\left\langle\left\langle T_{\Sigma}(X)\right\rangle\right\rangle, 1 \leq i \leq k$.

Theorem 2.3 Suppose that $A$ is a commutative semiring and $D$ is a distributive $\Sigma$-algebra equipped with a scalar multiplication $A \times D \rightarrow D,(a, d) \mapsto a d$, which satisfies the equations (1)-(6). Then any function $\varphi: X \rightarrow D$ extends to a unique distributive $\Sigma$-algebra morphism $\varphi^{\sharp}: A\left\langle T_{\Sigma}(X)\right\rangle \rightarrow D$ preserving scalar multiplication.

Proof. It is well-known that $\varphi$ extends to a unique $\Sigma$-algebra morphism $\bar{\varphi}$ : $T_{\Sigma}(X) \rightarrow D$. We further extend $\bar{\varphi}$ to $\varphi^{\sharp}$ by defining

$$
\varphi^{\sharp}(s)=\sum_{t \in T_{\Sigma}(X)}(s, t) \varphi(t),
$$


for all $s \in A\left\langle T_{\Sigma}(X)\right\rangle$. It is a routine matter to show that $\varphi^{\sharp}$ extends $\varphi$ and is a distributive $\Sigma$-algebra morphism that preserves scalar multiplication. Since the definition of $\varphi^{\sharp}$ was forced, the extension is unique.

A similar result holds when $A$ is a complete semiring, so that $A\left\langle\left\langle T_{\Sigma}(X)\right\rangle\right\rangle$ is a complete distributive $\Sigma$-algebra.

Theorem 2.4 Suppose that $A$ is a complete commutative semiring and $D$ is a complete distributive $\Sigma$-algebra equipped with a scalar multiplication $A \times D \rightarrow D$, $(a, d) \mapsto a d$, which satisfies the equations (1)-(6). Moreover, assume that

$$
\begin{aligned}
\left(\sum_{i \in I} a_{i}\right) d & =\sum_{i \in I} a_{i} d \\
a \sum_{i \in I} d_{i} & =\sum_{i \in I} a d_{i},
\end{aligned}
$$

for all $a, a_{i} \in A$ and $d, d_{i} \in D, i \in I$, where $I$ is any index set. Then any function $\varphi: X \rightarrow D$ extends to a unique complete distributive $\Sigma$-algebra morphism $\varphi^{\sharp}: A\left\langle\left\langle T_{\Sigma}(X)\right\rangle\right\rangle \rightarrow D$ preserving scalar multiplication.

When $A$ is ordered by $\leq$, we may order $A\left\langle\left\langle T_{\Sigma}(X)\right\rangle\right\rangle$, and thus $A\left\langle T_{\Sigma}(X)\right\rangle$, by the pointwise order: We define $s \leq s^{\prime}$ for $s, s^{\prime} \in A\left\langle\left\langle T_{\Sigma}(X)\right\rangle\right\rangle$ iff $(s, t) \leq\left(s^{\prime}, t\right)$ for all $t \in T_{\Sigma}(X)$. Equipped with this order, both $A\left\langle\left\langle T_{\Sigma}(X)\right\rangle\right\rangle$ and $A\left\langle T_{\Sigma}(X)\right\rangle$ are ordered distributive $\Sigma$-algebras. Moreover, scalar multiplication preserves the order in both arguments. Eventually, when $A$ is a continuous semiring, then $A\left\langle\left\langle T_{\Sigma}(X)\right\rangle\right\rangle$ is also continuous, and scalar multiplication preserves least upper bounds of directed sets in both arguments.

Corollary 2.5 Suppose that $A$ is an ordered commutative semiring and $D$ is an ordered distributive $\Sigma$-algebra equipped with an order preserving scalar multiplication $A \times D \rightarrow D,(a, d) \mapsto a d$, which satisfies the equations (1)-(6). Then any function $\varphi: X \rightarrow D$ extends to a unique distributive $\Sigma$-algebra morphism $\varphi^{\sharp}: A\left\langle T_{\Sigma}(X)\right\rangle \rightarrow D$ preserving scalar multiplication. Moreover, when $A$ is a continuous commutative semiring and $D$ is a continuous distributive $\Sigma$-algebra equipped with a continuous scalar multiplication $A \times D \rightarrow D,(a, d) \mapsto a d$, which satisfies the above equations, then any function $\varphi: X \rightarrow D$ extends to a unique continuous distributive $\Sigma$-algebra morphism $\varphi^{\sharp}: A\left\langle\left\langle T_{\Sigma}(X)\right\rangle\right\rangle \rightarrow D$ preserving scalar multiplication.

In the sequel, $A$ will denote a continuous (complete) commutative semiring where sums are defined by Proposition 2.1. Let $s$ be a formal tree series in $A\left\langle\left\langle T_{\Sigma}(X)\right\rangle\right\rangle$, and let $D$ denote a continuous distributive $\Sigma$-algebra equipped with a scalar multiplication $A \times D \rightarrow A$ satisfying (1)-(6) which is also continuous. The set $D^{X}$ of all functions $X \rightarrow D$ is also a continuous distributive $\Sigma$-algebra by the pointwise operations and ordering as is the the set of all continuous functions $D^{X} \rightarrow D$. Moreover, it is equipped with the pointwise scalar multiplication 
which again satisfies (1)-(6) and is continuous. Now $s$ induces a mapping $s^{D}$ : $D^{X} \rightarrow D, h \mapsto h^{\sharp}(s)$ for $h \in D^{X}$.

Proposition 2.6 The function $s^{D}$ is continuous. Moreover, the assignment $s \rightarrow s^{D}$ defines a continuous function of $s$.

Proof. It is known that when $t \in T_{\Sigma}(X)$, then the function $t^{D}: D^{X} \rightarrow D$ induced by $t$ is continuous, since it can be constructed from continuous functions (namely, the projections and the continuous operations of $D$ corresponding to the symbols in $\Sigma$ ) by function composition, see, e.g., Guessarian [31]. Since scalar multiplication and + are continuous, so is any function induced by a series in $A\left\langle T_{\Sigma}(X)\right\rangle$. But $s^{D}$ is the pointwise supremum of the functions induced by the polynomials $\sum_{t \in F}(s, t) t$, where $F$ is a finite subset of $T_{\Sigma}(X)$. Since the pointwise supremum of continuous functions is continuous, see Guessarian [31], the result follows.

To show that the assignment $s \mapsto s^{D}$ defines a continuous function, let $S$ denote a directed set in $A\left\langle\left\langle T_{\Sigma}(X)\right\rangle\right\rangle$. We need to prove that

$$
\left(\sup _{s \in S} s\right)^{D}=\sup _{s \in S} s^{D} .
$$

But for any $h: X \rightarrow D$,

$$
\begin{aligned}
\left.\operatorname{(sup}_{s \in S} s\right)^{D}(h) & =h^{\sharp}\left(\sup _{s \in S} s\right) \\
& =\sup _{s \in S} h^{\sharp}(s) \\
& =\sup _{s \in S} s^{D}(h) \\
& =\left(\sup _{s \in S} s^{D}\right)(h) .
\end{aligned}
$$

¿From now on we will write just $h$ for $h^{\sharp}$ and denote $s^{D}$ by just $s$.

In particular, formal tree series induce continuous mappings called substitutions as follows. Let $Y$ denote a nonempty set of variables, where $Y \cap(\Sigma \cup X)=$ $\emptyset$, and consider a mapping $h: Y \rightarrow A\left\langle\left\langle T_{\Sigma}(X \cup Y)\right\rangle\right\rangle$. This mapping can be extended to a mapping $h: T_{\Sigma}(X \cup Y) \rightarrow A\left\langle\left\langle T_{\Sigma}(X \cup Y)\right\rangle\right\rangle$ by $h(x)=x, x \in X$. Now, by the above result, for any series $s \in A\left\langle\left\langle T_{\Sigma}(X \cup Y)\right\rangle\right\rangle$, the mapping $h \mapsto h(s)$ is a continuous function of $h$. By the arguments outlined above, $h(s)$ can be constructed as follows. First, extend $h$ to trees by defining

$$
\begin{aligned}
& h\left(\omega\left(t_{1}, \ldots, t_{k}\right)\right)=\bar{\omega}\left(h\left(t_{1}\right), \ldots, h\left(t_{k}\right)\right)= \\
& \sum_{t_{1}^{\prime}, \ldots, t_{k}^{\prime} \in T_{\Sigma}(X \cup Y)}\left(h\left(t_{1}\right), t_{1}^{\prime}\right) \ldots\left(h\left(t_{k}\right), t_{k}^{\prime}\right) \omega\left(t_{1}^{\prime}, \ldots, t_{k}^{\prime}\right),
\end{aligned}
$$

for $\omega \in \Sigma_{k}$ and $t_{1}, \ldots, t_{k} \in T_{\Sigma}(X \cup Y), k \geq 0$. One more extension of $h$ yields a mapping $h: A\left\langle\left\langle T_{\Sigma}(X \cup Y)\right\rangle\right\rangle \rightarrow A\left\langle\left\langle T_{\Sigma}(X \cup Y)\right\rangle\right\rangle$ by defining $h(s)=$ 
$\sum_{t \in T_{\Sigma}(X \cup Y)}(s, t) h(t)$. Now $s(h)$ is just the value of this extended function on $s$. If $Y=\left\{y_{1}, \ldots, y_{n}\right\}$ is finite, we use the following notation: $h: Y \rightarrow$ $A\left\langle\left\langle T_{\Sigma}(X \cup Y)\right\rangle\right\rangle$, where $h\left(y_{i}\right)=s_{i}, 1 \leq i \leq n$, is denoted by $\left(s_{i}, 1 \leq i \leq n\right)$ or $\left(s_{1}, \ldots, s_{n}\right)$ and the value of $s$ with $\operatorname{argument} h$ is denoted by $s\left(s_{i}, 1 \leq i \leq n\right)$ or $s\left(s_{1}, \ldots, s_{n}\right)$. Intuitively, this is simply the substitution of the formal tree series $s_{i} \in A\left\langle\left\langle T_{\Sigma}(X \cup Y)\right\rangle\right\rangle$ into the variables $y_{i}, 1 \leq i \leq n$, of $s \in A\left\langle\left\langle T_{\Sigma}(X \cup Y)\right\rangle\right\rangle$. By Proposition 2.6, the mapping $s:\left(A\left\langle\left\langle T_{\Sigma}(X \cup Y)\right\rangle\right\rangle\right)^{Y} \rightarrow A\left\langle\left\langle T_{\Sigma}(X \cup Y)\right\rangle\right\rangle$, i. e., the substitution of formal tree series into the variables of $Y$, is a continuous mapping. Moreover, $s\left(s_{1}, \ldots, s_{n}\right)$ is also continuous in $s$. (So it is continuous in $s$ and in each $s_{i}$.) Observe that $s\left(s_{1}, \ldots, s_{n}\right)=\sum_{t \in T_{\Sigma}(X \cup Y)}(s, t) t\left(s_{1}, \ldots, s_{n}\right)$.

In certain situations, formulae are easier to read if we use the notation $s\left[s_{i} / y_{i}, 1 \leq i \leq n\right]$ for the substitution of the formal tree series $s_{i}$ into the variables $y_{i}, 1 \leq i \leq n$, of $s$ instead of the notation $s\left(s_{i}, 1 \leq i \leq n\right)$. So we will use sometimes this notation $s\left[s_{i} / y_{i}, 1 \leq i \leq n\right]$.

In the same way, $s \in A\left\langle\left\langle T_{\Sigma}(X \cup Y)\right\rangle\right\rangle$ also induces a mapping $s:\left(A\left\langle\left\langle T_{\Sigma}(X)\right\rangle\right\rangle\right)^{Y}$ $\rightarrow A\left\langle\left\langle T_{\Sigma}(X)\right\rangle\right\rangle$.

Our substitution on formal tree series is a generalization of the OI-substitutions on formal tree languages. We do not consider generalizations of the IO-substitution. Bozapalidis [11], Engelfriet, Fülöp, Vogler [19] and Fülöp, Vogler [23] consider these generalizations to formal tree series.

The construction of tree series and the above freeness results can be generalized to a great extent. Suppose that $D$ is any $\Sigma$-algebra and $A$ is any complete semiring. Then the set of functions $A \rightarrow D$, denoted $A\langle\langle D\rangle\rangle$, is a complete distributive $\Sigma$-algebra. We call the elements of $A\langle\langle D\rangle\rangle$ series and denote them as $\sum_{d \in D}(s, d) d$, or $\sum_{d \in \operatorname{supp}(s)}(s, d) d$. The sum of any family of series is their pointwise sum. The zero series serves as zero. Moreover, for each $\omega \in \Sigma_{k}, k \geq 1$, and for each $s_{1}, \ldots, s_{k} \in A\langle\langle D\rangle\rangle$,

$$
\omega\left(s_{1}, \ldots, s_{k}\right)=\sum_{d \in D}\left(\sum_{d=\omega\left(d_{1}, \ldots, d_{k}\right)}\left(s_{1}, d_{1}\right) \ldots\left(s_{k}, d_{k}\right)\right) d .
$$

Note also that $A\langle\langle D\rangle\rangle$ is equipped with a scalar multiplication $A \times A\langle\langle D\rangle\rangle \rightarrow$ $A\langle\langle D\rangle\rangle$. Note that equations (1)-(6) and (7), (8) hold. When $A$ is a continuous semiring then, equipped with the pointwise order, $A\langle\langle D\rangle\rangle$ is a continuous distributive $\Sigma$-algebra and scalar multiplication is continuous. We are now ready to state the promised generalization of Theorem 2.4.

Theorem 2.7 Suppose that $A$ is a complete commutative semiring and $D^{\prime}$ is a distributive $\Sigma$-algebra equipped with a scalar multiplication $A \times D^{\prime} \rightarrow D^{\prime}$ which satisfies the equations (1)-(6) as well as (7) and (8). Moreover, assume that $D$ is a $\Sigma$-algebra. Then any $\Sigma$-algebra morphism $\varphi: D \rightarrow D^{\prime}$ extends to a unique complete distributive $\Sigma$-algebra morphism $\varphi^{\sharp}: A\langle\langle D\rangle\rangle \rightarrow D^{\prime}$ preserving scalar multiplication. When $A$ is a continuous commutative semiring and $D^{\prime}$ is a continuous distributive $\Sigma$-algebra and the scalar multiplication $A \times D^{\prime} \rightarrow D^{\prime}$ is continuous, then so is the function $\varphi^{\sharp}$. 
Theorem 2.3 can be generalized in the same way. For more on series over $\Sigma$-algebras we refer the reader to Kuich [42, 44].

In the sequel, we shall make use of some basic facts about least fixed points of continuous functions that we review next.

A complete partially ordered set, or cpo, for short, is a partially ordered set $P$ which has a bottom element, usually denoted $\perp$, such that $\sup D$ exists for each directed set $D \subseteq P$. Note that continuous monoids and continuous distributive $\Sigma$-algebras are cpo's. When $P$ and $Q$ are cpo's, a function $f: P \rightarrow Q$ is called continuous if $f$ preserves the least upper bound of directed sets (see also above). It is clear that any composition of continuous functions is continuous, and any direct product of cpo's is a cpo equipped with the pointwise order. Moreover, when $P, Q$ are cpo's, the set of continuous functions $P \rightarrow Q$ equipped with the pointwise order is also a cpo.

Suppose that $P$ and $Q_{i}, i \in I$ are cpo's and let $\prod_{i \in I} Q_{i}$ denote the direct product of the $Q_{i}$. Then for any $j \in I$, the $j$ th projection function $\prod_{i \in I} Q_{i} \rightarrow Q_{j}$ is continuous. Moreover, a function $f: P \rightarrow \prod_{i \in I} Q_{i}$ is continuous iff each "component function" $f_{i}: P \rightarrow Q_{i}$ is continuous. And when $I$ is finite, say $I=\{1, \ldots, n\}$, then a function $f: \prod_{i \in I} Q_{i} \rightarrow P$ is continuous iff it is continuous separately in each argument, i.e., when

$$
f\left(a_{1}, \ldots, \sup D, \ldots, a_{n}\right)=\sup f\left(a_{1}, \ldots, D, \ldots, a_{n}\right)
$$

holds for each $1 \leq i \leq n, a_{j} \in P_{j}, j \neq i$, and for each directed set $D \subseteq P_{i}$. In the sequel, we will use these facts without any further mention.

Due to a well-known fixed point theorem, that we recall now, cpo's and continuous functions have been used widely to give semantics to recursive definitions, see, e.g., Bloom, Ésik [6], Guessarian [31].

Theorem 2.8 Suppose that $P$ and $Q$ are cpo's and $f$ is a continuous function $P \times Q \rightarrow P$. Then for each $q \in Q$ there is a least $p \in P$ with $p=f(p, q)$, called the least fixed point of $f$ with respect to the parameter $q$. Moreover, the function $Q \rightarrow P$ that takes $q$ to the least fixed point $p$ is continuous.

In fact, a similar result holds not only for continuous functions, but for any order preserving function $f: P \times Q \rightarrow P$. But when $f$ is continuous, the least fixed point can be constructed as the least upper bound $\sup p_{n}$, where $p_{0}=\perp$ and $p_{n+1}=f\left(p_{n}, q\right)$, for all $n \geq 0$. (The set $\left\{p_{n} \mid n \geq 0\right\}$ is a chain, and is thus directed.)

In Bloom, Ésik [6], the function $Q \rightarrow P$ arising from Theorem 2.8 that provides the parameterized least fixed point for a given continuous function $f: P \times Q \rightarrow P$ is denoted $f^{\dagger}$. Here, we will mainly use the notation $\mu x . f(x, y)$ or, for $f: P \rightarrow P$, also fix $(f)$.

We now recall three very important elementary facts about least fixed points of continuous functions. Theorem 2.9 is independently due to Bekić [3] and De Bakker, Scott [16]. For Proposition 2.10, see also Niwiński [49]. 
Theorem 2.9 Suppose that $f: P \times Q \times R \rightarrow P$ and $g: P \times Q \times R \rightarrow Q$ are continuous functions, where $P, Q, R$ are cpo's. Let $h: P \times Q \times R \rightarrow P \times Q$ denote the "target pairing" of $f$ and $g$, so that $h(x, y, z)=(f(x, y, z), g(x, y, z))$. Then

$$
\mu(x, y) . h(x, y, z)=(\mu x . f(x, k(x, z), z), k(\mu x . f(x, k(x, z), z), z))
$$

where $k(x, z)=\mu y \cdot g(x, y, z)$.

Proposition 2.10 Suppose that $f: P \times P \times Q \rightarrow P$ is a continuous function, where $P$ and $Q$ are cpo's. Then

$$
\mu x . \mu y \cdot f(x, y, z)=\mu x \cdot f(x, x, z) .
$$

Proposition 2.11 Suppose that $f: P \times Q \rightarrow P$ and $g: R \rightarrow Q$ are continuous functions, where $P, Q, R$ are all cpo's. Then

$$
\mu x . f(x, g(z))=h(g(z)),
$$

where $h(y)=\mu x . f(x, y)$.

We refer to the equation in Theorem 2.9 as the Bekić-De Bakker-Scott rule. The equation in Proposition 2.10 is usually referred to as the diagonal equation, or the double iteration equation. In the terminology of Bloom, Ésik [6], Proposition 2.11 asserts that the parameter identity holds.

The above results describe three equational properties of the least fixed point operation on continuous functions. For a complete description, we refer the reader to Bloom, Ésik [6]. Least fixed points of continuous functions on cpo's are also least pre-fixed points. In Ésik [21], it is shown that the equational properties of the least fixed point operation on continuous functions on cpo's are exactly the same as that of the least pre-fixed point operation on ordered preserving functions on partially ordered sets in general.

In the sequel, $Y, Y^{\prime}, Z$ will denote sets of variables that are disjoint from $\Sigma$ and $X$, and $Y_{k}, k \geq 1$, will denote the set of variables $\left\{y_{1}, \ldots, y_{k}\right\}$. Moreover, $Y_{0}=\emptyset$. Furthermore, $I$ and $I^{\prime}$ will denote arbitrary index sets.

Given a set $S, S^{I_{1} \times I_{2}}$ will denote in the sequel the set of matrices indexed by $I_{1} \times I_{2}$ with entries in $S$. (E. g., $\left(A\left\langle\left\langle T_{\Sigma}(X)\right\rangle\right\rangle\right)^{I^{\prime} \times I^{k}}$ denotes the set of matrices $M$, such that the $\left(i,\left(i_{1}, \ldots, i_{k}\right)\right)$-entry of $M$ is in $A\left\langle\left\langle T_{\Sigma}(X)\right\rangle\right\rangle, i \in I^{\prime}, i_{1}, \ldots, i_{k} \in I$.)

Our tree automata will be defined by transition matrices. A matrix $M \in$ $\left(A\left\langle\left\langle T_{\Sigma}\left(X \cup Y_{k}\right)\right\rangle\right\rangle\right)^{I^{\prime} \times I^{k}}, k \geq 1, I^{\prime}$ and $I$ arbitrary index sets, induces a mapping $M:\left(A\left\langle\left\langle T_{\Sigma}\left(X \cup Y^{\prime}\right)\right\rangle\right\rangle\right)^{I \times 1} \times \ldots \times\left(A\left\langle\left\langle T_{\Sigma}\left(X \cup Y^{\prime}\right)\right\rangle\right)^{I \times 1} \rightarrow\left(A\left\langle\left\langle T_{\Sigma}\left(X \cup Y^{\prime}\right)\right\rangle\right\rangle\right)^{I^{\prime} \times 1}\right.$

(there are $k$ argument vectors), defined by the entries of the resulting vector as follows: For $P_{1}, \ldots, P_{k} \in\left(A\left\langle\left\langle T_{\Sigma}\left(X \cup Y^{\prime}\right)\right\rangle\right\rangle\right)^{I \times 1}$ we define, for all $i \in I^{\prime}$,

$$
\begin{aligned}
& M\left(P_{1}, \ldots, P_{k}\right)_{i}=\sum_{i_{1}, \ldots, i_{k} \in I} M_{i,\left(i_{1}, \ldots, i_{k}\right)}\left(\left(P_{1}\right)_{i_{1}}, \ldots,\left(P_{k}\right)_{i_{k}}\right)= \\
& \sum_{i_{1}, \ldots, i_{k} \in I} \sum_{t \in T_{\Sigma}\left(X \cup Y_{k}\right)}\left(M_{i,\left(i_{1}, \ldots, i_{k}\right)}, t\right) t\left(\left(P_{1}\right)_{i_{1}}, \ldots,\left(P_{k}\right)_{i_{k}}\right) .
\end{aligned}
$$


Theorem 2.12 Let $M \in\left(A\left\langle\left\langle T_{\Sigma}\left(X \cup Y_{k}\right)\right\rangle\right\rangle\right)^{I^{\prime} \times I^{k}}, k \geq 1$. Define $\bar{M} \in\left(A\left\langle\left\langle T_{\Sigma}(X \cup\right.\right.\right.$ $\left.\left.\left.\left.Y_{k}\right)\right\rangle\right\rangle\right)^{I^{\prime} \times I^{m}}, m>k$, by

$$
\bar{M}_{i,\left(i_{1}, \ldots, i_{m}\right)}=\delta_{i, i_{m}} \delta_{i_{m}, i_{m-1}} \cdots \delta_{i_{k+2}, i_{k+1}} M_{i,\left(i_{1}, \ldots, i_{k}\right)},
$$

for $i \in I^{\prime}, i_{1}, \ldots, i_{m} \in I$.

Then, for $P_{1}, \ldots, P_{m} \in\left(A\left\langle\left\langle T_{\Sigma}\left(X \cup Y^{\prime}\right)\right\rangle\right\rangle\right)^{I \times 1}$,

$$
\bar{M}\left(P_{1}, \ldots, P_{m}\right)=M\left(P_{1}, \ldots, P_{k}\right) .
$$

Proof.

$$
\begin{aligned}
& \bar{M}\left(P_{1}, \ldots, P_{m}\right)_{i}= \\
& \sum_{i_{1}, \ldots, i_{m} \in I} \bar{M}_{i,\left(i_{1}, \ldots, i_{m}\right)}\left(\left(P_{1}\right)_{i_{1}}, \ldots,\left(P_{m}\right)_{i_{m}}\right)= \\
& \sum_{i_{1}, \ldots, i_{m} \in I} \delta_{i, i_{m}} \delta_{i_{m}, i_{m-1}} \cdots \delta_{i_{k+2}, i_{k+1}} M_{i,\left(i_{1}, \ldots, i_{k}\right)}\left(\left(P_{1}\right)_{i_{1}}, \ldots,\left(P_{k}\right)_{i_{k}}\right)= \\
& \sum_{i_{1}, \ldots, i_{k} \in I} M_{i,\left(i_{1}, \ldots, i_{k}\right)}\left(\left(P_{1}\right)_{i_{1}}, \ldots,\left(P_{k}\right)_{i_{k}}\right)= \\
& M\left(P_{1}, \ldots, P_{k}\right)_{i}, \quad i \in I^{\prime} .
\end{aligned}
$$

For the definition of the tree series transducers we will need a generalization of the substitution defined by a matrix in $\left(A\left\langle\left\langle T_{\Sigma}\left(X \cup Y_{k}\right)\right\rangle\right\rangle\right)^{I^{\prime} \times I^{k}}, k \geq 1$. A matrix $M \in\left(A\left\langle\left\langle T_{\Sigma}\left(X \cup Y_{m}\right)\right\rangle\right\rangle\right)^{I^{\prime} \times\left(I \times Z_{k}\right)^{m}}, Z_{k}=\left\{z_{1}, \ldots, z_{k}\right\}, k \geq 1$, induces a mapping

$M:\left(A\left\langle\left\langle T_{\Sigma}\left(X \cup Y^{\prime}\right)\right\rangle\right\rangle\right)^{I \times 1} \times \cdots \times\left(A\left\langle\left\langle T_{\Sigma}\left(X \cup Y^{\prime}\right)\right\rangle\right\rangle\right)^{I \times 1} \rightarrow\left(A\left\langle\left\langle T_{\Sigma}\left(X \cup Y^{\prime}\right)\right\rangle\right\rangle\right)^{I^{\prime} \times 1}$

(there are $k$ argument vectors) defined by the entries of the resulting vector as follows: For $P_{1}, \ldots, P_{k} \in\left(A\left\langle\left\langle T_{\Sigma}\left(X \cup Y^{\prime}\right)\right\rangle\right\rangle\right)^{I \times 1}$ we define, for all $i \in I^{\prime}$,

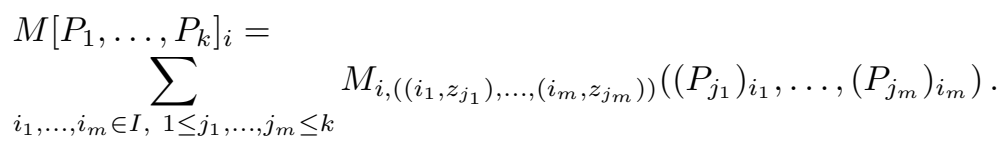

Theorem 2.13 Let $C=\left\{\left(\left(i_{1}, z_{1}\right), \ldots,\left(i_{k}, z_{k}\right)\right) \mid i_{1}, \ldots, i_{k} \in I\right\}$ and $M \in$ $\left(A\left\langle\left\langle T_{\Sigma}\left(X \cup Y_{k}\right)\right\rangle\right\rangle\right)^{I^{\prime} \times\left(I \times Z_{k}\right)^{k}}, k \geq 1$, such that $M_{i, \alpha}=0$ for $i \in I^{\prime}$ and $\alpha \in(I \times$ $\left.Z_{k}\right)^{k}-C$. Define $\bar{M} \in\left(A\left\langle\left\langle T_{\Sigma}\left(X \cup Y_{k}\right)\right\rangle\right\rangle\right)^{I^{\prime} \times I^{k}}$ by $\bar{M}_{i,\left(i_{1}, \ldots, i_{k}\right)}=M_{i,\left(\left(i_{1}, z_{1}\right), \ldots,\left(i_{k}, z_{k}\right)\right)}$ for $i \in I^{\prime}, i_{1}, \ldots, i_{k} \in I$. Then, for $P_{1}, \ldots, P_{k} \in\left(A\left\langle\left\langle T_{\Sigma}\left(X \cup Y^{\prime}\right)\right\rangle\right\rangle\right)^{I \times 1}$,

$$
M\left(P_{1}, \ldots, P_{k}\right)=\bar{M}\left[P_{1}, \ldots, P_{k}\right] .
$$

Proof.

$$
\begin{aligned}
& M\left[P_{1}, \ldots, P_{k}\right]_{i}= \\
& \sum_{i_{1}, \ldots, i_{k} \in I} M_{i,\left(\left(i_{1}, z_{1}\right), \ldots,\left(i_{k}, z_{k}\right)\right)}\left(\left(P_{1}\right)_{i_{1}}, \ldots,\left(P_{k}\right)_{i_{k}}\right)= \\
& \sum_{i_{1}, \ldots, i_{k} \in I} \bar{M}_{i,\left(i_{1}, \ldots, i_{k}\right)}\left(\left(P_{1}\right)_{i_{1}}, \ldots,\left(P_{k}\right)_{i_{k}}\right)= \\
& \bar{M}\left(P_{1}, \ldots, P_{k}\right)_{i}, \quad i \in I^{\prime} .
\end{aligned}
$$




\section{Tree automata and systems of equations}

In this section we define tree automata and systems of equations (over semirings). These notions are a framework for the consideration of finite tree automata and pushdown tree automata, and polynomial systems. The definitions are slightly adapted from Kuich [38]. The main result of this section is that (finite, polynomial) tree automata and (finite, polynomial) systems are equivalent mechanisms.

Our tree automata are a generalization of the nondeterministic root-tofrontier tree recognizers. (See Gécseg, Steinby [24, 25] and Kuich [38].) A tree automaton (with input alphabet $\Sigma$ and leaf alphabet $X$ over the semiring A)

$$
\mathfrak{A}=(I, M, S, P)
$$

is given by

(i) a nonempty set $I$ of states,

(ii) a transition matrix $M \in\left(A\left\langle\left\langle T_{\Sigma}\left(X \cup Y_{m}\right)\right\rangle\right\rangle\right)^{I \times I^{m}}$, for some $m \geq 1$,

(iii) a row finite row vector $S \in\left(A\left\langle\left\langle T_{\Sigma}\left(X \cup Y_{1}\right)\right\rangle\right\rangle\right)^{1 \times I}$, called the initial state vector,

(iv) a column vector $P \in\left(A\left\langle\left\langle T_{\Sigma}(X)\right\rangle\right\rangle\right)^{I \times 1}$, called the final state vector.

The approximation sequence $\left(\sigma^{j} \mid j \in \mathbb{N}\right), \sigma^{j} \in\left(A\left\langle\left\langle T_{\Sigma}(X)\right\rangle\right\rangle\right)^{I \times 1}, j \geq 0$, associated with $\mathfrak{A}$ is defined as follows:

$$
\sigma^{0}=0, \quad \sigma^{j+1}=M\left(\sigma^{j}, \ldots, \sigma^{j}\right)+P, \quad j \geq 0 .
$$

(There are $m$ argument vectors $\sigma^{j}$.) The behavior $\|\mathfrak{A}\| \in A\left\langle\left\langle T_{\Sigma}(X)\right\rangle\right\rangle$ of the tree automaton $\mathfrak{A}$ is defined by

$$
\|\mathfrak{A}\|=\sum_{i \in I} S_{i}\left(\sigma_{i}\right)=S(\sigma),
$$

where $\sigma \in\left(A\left\langle\left\langle T_{\Sigma}(X)\right\rangle\right\rangle\right)^{I \times 1}$ is the least upper bound of the approximation sequence associated with $\mathfrak{A}$. Since $\sigma^{j} \leq \sigma^{j+1}$ for all $j$, and since $\left(A\left\langle\left\langle T_{\Sigma}(X \cup\right.\right.\right.$ $\left.\left.\left.\left.Y_{m}\right)\right\rangle\right\rangle\right)^{I \times I^{m}}$ has all directed least upper bounds with respect to the pointwise order, it follows that this least upper bound and, hence, the behavior of $\mathfrak{A}$ exist.

A tree automaton $\mathfrak{A}=(I, M, S, P)$ is called finite iff $I$ is finite. A tree automaton $\mathfrak{A}=(I, M, S, P)$ is called simple iff the entries of the transition matrix $M$, of the initial state vector $S$ and of the final state vector $P$ have the following specific form:

(i) the entries of $M$ are of the form $\sum_{1 \leq k \leq m} \sum_{\omega \in \Sigma_{k}} a_{\omega} \omega\left(y_{1}, \ldots, y_{k}\right)+$ $\sum_{\omega \in \Sigma_{0} \cup X} a_{\omega} \omega+a y_{1}, a_{\omega}, a \in A$ 
(ii) the entries of $P$ are of the form $\sum_{\omega \in \Sigma_{0} \cup X} a_{\omega} \omega, a_{\omega} \in A$;

(iii) the entries of $S$ are of the form $d y_{1}, d \in A$.

It is called proper iff there are no terms $a y_{1}$ in (i). Observe that the term $a y_{1}$ in (i) corresponds to $\varepsilon$-moves in ordinary automata.

Intuitively, a simple tree automaton $\mathfrak{A}$ recognizes a tree $t \in T_{\Sigma}(X)$ with coefficient $(\| \mathfrak{A}||, t)$ as follows in a nondeterministic way.

At the root of $t, \mathfrak{A}$ may be in any initial state $i \in I$, i. e., in a state with $(S, i) \neq 0$. We now describe a computation starting in the initial state $i_{0} \in I$ and its weight. Consider any node of $t$ labelled by $\omega \in \Sigma_{k}, k \geq 1$. If in the recognition procedure $\mathfrak{A}$ is in state $i$ and $\left(M_{i,\left(i_{1}, \ldots, i_{m}\right)}, \omega\left(y_{1}, \ldots, y_{k}\right)\right)=a_{i} \neq 0$ then $\mathfrak{A}$ proceeds in parallel at states $i_{1}, \ldots, i_{k}$ at the roots of $t_{1}, \ldots, t_{m}$, respectively. Consider any node of $t$ labelled by $\omega \in \Sigma_{0} \cup X$. If, in the recognition procedure, $\mathfrak{A}$ is in state $i$ and $\left(P_{i}, \omega\right)=a_{i} \neq 0$ or $\left(M_{i,\left(i_{1}, \ldots, i_{m}\right)}, \omega\right)=a_{i} \neq 0$ for some $i_{1}, \ldots, i_{m} \in I$, then $\mathfrak{A}$ terminates this branch of its computation. If, in the recognition procedure, $\mathfrak{A}$ is in state $i$ and $\left(M_{i,\left(i_{1}, \ldots, i_{m}\right)}, y_{1}\right)=a_{i} \neq 0$ then $\mathfrak{A}$ moves to state $i_{1}$.

The weight of such a computation starting in the initial state $i_{0}$ is $\left(S, i_{0}\right)$ multiplied with all the semiring elements $a_{i}$ occuring in the procedure described above. The coefficient $(\|\mathfrak{A}\|, t)$ is then the sum of all weights of all possible computations.

In Kuich [38], tree automata were defined by a sequence of transition matrices. But, essentially, these tree automata are equivalent to our tree automata by Theorem 2.12 .

Consider the case that $A$ is the semiring $\mathbb{N}^{\infty}$, i. e., we consider tree series in $\mathbb{N}^{\infty}\left\langle\left\langle T_{\Sigma}(X)\right\rangle\right\rangle$. A simple tree automaton is called 1-simple iff all the coefficients $a_{\omega}, a$ in (i), $a_{\omega}$ in (ii) and $d$ in (iii) belong to $\{0,1\}$. By Seidl [55], Proposition 3.1, the coefficient $(\|\mathfrak{A}\|, t)$ of the behavior of $\mathfrak{A}$ is the number (possible $\infty$ ) of distinct computations for $t$.

Theorem 3.1 Consider a 1-simple tree automaton $\mathfrak{A}$ and let $d(t), t \in T_{\Sigma}(X)$, be the number (possibly $\infty$ ) of distinct computations of $\mathfrak{A}$ for $t$. Then

$$
\|\mathfrak{A}\|=\sum_{t \in T_{\Sigma}(X)} d(t) t \in \mathbb{N}^{\infty}\left\langle\left\langle T_{\Sigma}(X)\right\rangle\right\rangle .
$$

We now turn to systems.

A system (with variables in $Z=\left\{z_{i} \mid i \in I\right\}$ ) is a system of formal equations $z_{i}=p_{i}, i \in I, I$ an arbitrary index set, where each $p_{i}$ is in $A\left\langle\left\langle T_{\Sigma}\left(X \cup Z_{i}\right)\right\rangle\right\rangle$. Here $Z_{i}$ is, for each $i \in I$, a finite subset of $Z$ with $\left|Z_{i}\right| \leq m$ for some $m \geq 0$. The system can be written in matrix notation as $z=p(z)$. Here $z$ and $p$ denote vectors, whose $i$-th component is $z_{i}$ and $p_{i}, i \in I$, respectively. A solution to the system $z=p(z)$ is given by $\sigma \in\left(A\left\langle\left\langle T_{\Sigma}(X)\right\rangle\right\rangle\right)^{I \times 1}$ such that $\sigma=p(\sigma)$. A solution $\sigma$ of $z=p(z)$ is called least solution iff $\sigma \leq \tau$ for all solutions $\tau$ of $z=p(z)$. 
The approximation sequence $\left(\sigma^{j} \mid j \in \mathbb{N}\right), \sigma^{j} \in\left(A\left\langle\left\langle T_{\Sigma}(X)\right\rangle\right\rangle\right)^{I \times 1}, j \geq 0$, associated with the system $z=p(z)$ is defined as follows:

$$
\sigma^{0}=0, \quad \sigma^{j+1}=p\left(\sigma^{j}\right), \quad j \geq 0 .
$$

Since $\sigma^{j} \leq \sigma^{j+1}$ for all $j$, and since $\left(A\left\langle\left\langle T_{\Sigma}(X)\right\rangle\right\rangle\right)^{I \times 1}$ has least upper bounds of all directed sets, the least upper bound $\sigma=\sup \left(\sigma^{j} \mid j \in \mathbb{N}\right)$ of this approximation sequence exists. Moreover, it is the least solution of the system $z=p(z)$.

Our systems are a generalization of the systems of linear equations of Berstel, Reutenauer [5]. A system $z_{i}=p_{i}, i \in I$, is called proper iff $\left(p_{i}, z_{j}\right)=0$ for all $i, j \in I$. It is called finite iff $I$ is finite.

Theorem 3.2 For each system there exists a proper one with the same least solution. A proper system has a unique solution.

Proof. The construction is as follows. Consider a system $z=p$ as defined above. Write it in the form $z=M z+r$, where $M \in A^{I \times I}$ and $\left(r_{i}, z_{j}\right)=0$ for $i, j \in I$. Then, by the diagonal identity (Proposition 2.10), the systems $z=M z+r$ and $z=M^{*} r$ have the same least solution and, by construction, $z=M^{*} r$ is a proper system. An adaption of the proof of Proposition 6.1 of Berstel, Reutenauer [5] proves the second sentence of our theorem. Clearly, this unique solution is at the same time the least solution.

We now show that tree automata and systems are mechanisms of equal power. For a given tree automaton $\mathfrak{A}=(I, M, S, P)$ as defined above we construct the system with variables in $Z=\left\{z_{i} \mid i \in I\right\}$

$$
z_{i}=\sum_{i_{1}, \ldots, i_{m} \in I} M_{i,\left(i_{1}, \ldots, i_{m}\right)}\left(z_{i_{1}}, \ldots, z_{i_{m}}\right)+P_{i}, \quad i \in I .
$$

Here we have substituted the variables $z_{i_{1}}, \ldots, z_{i_{m}}$ for the variables $y_{1}, \ldots, y_{m}$ in $M_{i,\left(i_{1}, \ldots, i_{m}\right)}\left(y_{1}, \ldots, y_{m}\right)$. In matrix notation, this system can be written as

$$
z=M(z, \ldots, z)+P .
$$

Here $z$ is an $I \times 1$-vector whose $i$-th component is the variable $z_{i}, i \in I$.

As before, the approximation sequences associated with this system and to the tree automaton $\mathfrak{A}$ coincide. Consider now the system with variables in $\left\{z_{0}\right\} \cup Z$

$$
z_{0}=\sum_{i \in I} S_{i}\left(z_{i}\right), \quad z=M(z, \ldots, z)+P
$$

Then the $z_{0}$-component of its least solution is equal to $\|\mathfrak{A}\|$.

Conversely, consider a system $z=p(z)$ as defined above. Let $Z_{i}=\left\{z_{i_{1}}, \ldots, z_{i_{k}}\right\}$, $i \in I$, and $p_{i}=p_{i}\left(z_{i_{1}}, \ldots, z_{i_{k}}\right), k \leq m$. Construct now the tree automaton $\mathfrak{A}=(Z, M, S, 0)$, where, for all $i, j_{1}, \ldots, j_{m} \in I$,

$$
M_{z_{i},\left(z_{j_{1}}, \ldots, z_{j_{m}}\right)}\left(y_{1}, \ldots, y_{m}\right)=\delta_{i, j_{m}} \delta_{j_{m}, j_{m-1}} \cdots \delta_{j_{k+2}, j_{k+1}} \delta_{j_{k}, i_{k}} \cdots \delta_{j_{1}, i_{1}} p_{i}\left(y_{1}, \ldots, y_{k}\right)
$$


Moreover, choose a $z_{i_{0}} \in Z$ and let $S_{z_{i_{0}}}\left(y_{1}\right)=y_{1}, S_{z_{i}}\left(y_{1}\right)=0$ for $z_{i} \neq z_{i_{0}}$.

Let $\left(\sigma^{j} \mid j \in \mathbb{N}\right)$ and $\left(\tau^{j} \mid j \in \mathbb{N}\right)$ be the approximation sequences associated to $z=p(z)$ and $\mathfrak{A}$, respectively. We claim that $\sigma^{j}=\tau^{j}$ for $j \geq 0$ and show it by induction on $j$. The case $j=0$ being clear, we proceed with $j>0$. Then we obtain, for all $i \in I$,

$$
\begin{aligned}
& \tau_{z_{i}}^{j}=M\left(\tau^{j-1}, \ldots, \tau^{j-1}\right)_{z_{i}}= \\
& \sum_{j_{1}, \ldots, j_{m} \in I} M_{z_{i},\left(z_{j_{1}}, \ldots, z_{j_{m}}\right)}\left(\tau_{z_{j_{1}}}^{j-1}, \ldots, \tau_{z_{j_{m}}}^{j-1}\right)= \\
& \sum_{j_{1}, \ldots, j_{m} \in I} \delta_{i, j_{m}} \delta_{j_{m}, j_{m-1}} \cdots \delta_{j_{k+2}, j_{k+1}} \delta_{j_{k}, i_{k}} \cdots \delta_{j_{1}, i_{1}} p_{i}\left(\tau_{z_{j_{1}}}^{j-1}, \ldots, \tau_{z_{j_{k}}}^{j-1}\right)= \\
& p_{i}\left(\tau_{z_{i_{1}}}^{j-1}, \ldots, \tau_{z_{i_{k}}}^{j-1}\right)=p_{i}\left(\sigma_{z_{i_{1}}}^{j-1}, \ldots, \sigma_{z_{i_{k}}}^{j-1}\right)=\sigma_{i}^{j} .
\end{aligned}
$$

Hence, $\|\mathfrak{A}\|$ is equal to the $z_{i_{0}}$-component of the least solution of $z=p(z)$.

Observe that we could place, for $k=0, p_{i}$ into $P_{z_{i}}$ instead into $M_{z_{i},\left(z_{i}, \ldots, z_{i}\right)}$. Theorem 3.3 summarizes the above considerations.

Theorem 3.3 A power series $s \in A\left\langle\left\langle T_{\Sigma}(X)\right\rangle\right\rangle$ is a component of the least solution of a system iff $s$ is the behavior of a tree automaton.

We now consider polynomial tree automata and polynomial systems and show that they are mechanisms of equal power.

A tree automaton $\mathfrak{A}=(I, M, S, P)$ is called polynomial iff the following conditions are satisfied:

(i) The entries of $M$ are polynomials in $A\left\langle T_{\Sigma}\left(X \cup Y_{m}\right)\right\rangle$.

(ii) The entries of the initial state vector $S$ are of the form $S_{i}=d_{i} y_{1}, d_{i} \in A$, $i \in I$.

(iii) The entries of the final state vector $P$ are polynomials in $A\left\langle T_{\Sigma}(X)\right\rangle$.

A system (with variables in $Z$ ) $z_{i}=p_{i}, i \in I$, is called polynomial iff each $p_{i}$ is a polynomial in $A\left\langle T_{\Sigma}\left(X \cup Z_{i}\right)\right\rangle, i \in I$.

The same constructions that proved Theorem 3.3 prove also the next theorem.

Theorem 3.4 A power series $s \in A\left\langle\left\langle T_{\Sigma}(X)\right\rangle\right\rangle$ is a component of the least solution of a polynomial system iff $s$ is the behavior of a polynomial tree automaton.

A system $z_{i}=p_{i}, i \in I$, is called simple iff $p_{i}$ is a sum of terms of the following form:

(i) $a \omega\left(z_{i_{1}}, \ldots, z_{i_{k}}\right), a \in A, \omega \in \Sigma_{k}, i_{1}, \ldots, i_{k} \in I, 1 \leq k \leq m$, for some $m \geq 1$,

(ii) $a \omega, a \in A, \omega \in \Sigma_{0} \cup X$,

(iii) $a z_{1}, a \in A$. 
Theorem 3.5 Let $s \in A\left\langle\left\langle T_{\Sigma}(X)\right\rangle\right\rangle$ be a component of the least solution of a finite polynomial system. Then there exists a finite polynomial system that is simple and proper such that $s$ is a component of its least solution.

Proof. By Theorem 3.2 and Lemma 6.3 of Berstel, Reutenauer [5].

Corollary 3.6 The following statements on a formal tree series in $A\left\langle\left\langle T_{\Sigma}(X)\right\rangle\right\rangle$ are equivalent:

(i) $s$ is a component of the least solution of a finite polynomial system;

(ii) $s$ is a component of the unique solution of a finite polynomial system that is also simple and proper;

(iii) $s$ is the behavior of a finite polynomial tree automaton;

(iv) $s$ is the behavior of a finite polynomial tree automaton with one initial state of weight 1 that is also simple and proper.

Proof. By Theorems 3.5 and 3.4, the statements (i), (ii) and (iii) are equivalent. By the construction in the proof of Theorem 3.3, statement (iv) is implied by statement (iii).

If a formal tree series in $A\left\langle\left\langle T_{\Sigma}(X)\right\rangle\right\rangle$ satisfies one and, hence, all statements of Corollary 3.6 we call it recognizable. The collection of all recognizable tree series in $A\left\langle\left\langle T_{\Sigma}(X)\right\rangle\right\rangle$ is denoted by $A^{\text {rec }}\left\langle\left\langle T_{\Sigma}(X)\right\rangle\right\rangle$. In the theory of tree languages, recognizable tree languages are defined only for finite alphabets $\Sigma$ and $X$. We allow also infinite alphabets $\Sigma$ and $X$. This is justified by the observation that, for $s \in A^{\text {rec }}\left\langle\left\langle T_{\Sigma}(X)\right\rangle\right\rangle$, there exist finite alphabets $\Sigma^{\prime}$ and $X^{\prime}, \Sigma^{\prime} \subseteq \Sigma, X^{\prime} \subseteq X$, such that $\operatorname{supp}(s) \subseteq T_{\Sigma^{\prime}}\left(X^{\prime}\right)$. Moreover

$$
A^{\mathrm{rec}}\left\langle\left\langle T_{\Sigma}(X)\right\rangle\right\rangle=\bigcup_{\Sigma^{\prime} \subseteq \Sigma \text { finite, } X^{\prime} \subseteq X \text { finite }} A^{\mathrm{rec}}\left\langle\left\langle T_{\Sigma^{\prime}}\left(X^{\prime}\right)\right\rangle\right\rangle .
$$

Example 3.1. ${ }^{1}$ (See Berstel, Reutenauer [5], Examples 6.2 and 4.2.) Our basic semiring is $\mathbb{Z}$, the semiring of integers. Let $\Sigma=\Sigma_{1} \cup \Sigma_{2}, \Sigma_{1}=\{\ominus\}, \Sigma_{2}=\{\oplus, \otimes\}$. We will evaluate arithmetic expressions with operators $\ominus, \oplus, \otimes$, and operands in the leaf alphabet $X$.

Define an interpretation eval of the elements of $X$, i. e., eval $: X \rightarrow \mathbb{Z}$. Extend it to a mapping eval $: T_{\Sigma}(X) \rightarrow \mathbb{Z}$ by eval $(\ominus(t))=-\operatorname{eval}(t), \operatorname{eval}\left(\oplus\left(t_{1}, t_{2}\right)\right)=$ $\operatorname{eval}\left(t_{1}\right)+\operatorname{eval}\left(t_{2}\right), \operatorname{eval}\left(\otimes\left(t_{1}, t_{2}\right)\right)=\operatorname{eval}\left(t_{1}\right) \cdot \operatorname{eval}\left(t_{2}\right)$ for $t, t_{1}, t_{2} \in T_{\Sigma}(X)$. Then eval $=\sum_{t \in T_{\Sigma}(X)} \operatorname{eval}(t) t$ is a formal tree series in $\mathbb{Z}\left\langle\left\langle T_{\Sigma}(X)\right\rangle\right.$.

Consider the proper system

$$
\begin{aligned}
& z_{1}=\oplus\left(z_{1}, z_{2}\right)+\oplus\left(z_{2}, z_{1}\right)+\otimes\left(z_{1}, z_{1}\right)+(-1) \ominus\left(z_{1}\right)+\sum_{x \in X} \operatorname{eval}(x) x, \\
& z_{2}=\oplus\left(z_{2}, z_{2}\right)+\otimes\left(z_{2}, z_{2}\right)+\ominus\left(z_{2}\right)+\sum_{x \in X} x .
\end{aligned}
$$

\footnotetext{
${ }^{1}$ In the examples we often refrain from our convention that the basic semiring is continuous.
} 
Let $\left(\sigma_{1}, \sigma_{2}\right)$ be its unique solution. Then we claim that $\sigma_{1}=$ eval, $\sigma_{2}=$ char, where char $=\sum_{t \in T_{\Sigma}(X)} t$. The claim is proven by substituting (eval, char) into the equations of the system:

$$
\begin{aligned}
& \oplus(\text { eval, char })+\oplus(\text { char, eval })+\oplus(\text { eval, eval })-\ominus(\text { eval })+\sum_{x \in \Sigma} \operatorname{eval}(x) x= \\
& \sum_{t_{1}, t_{2} \in T_{\Sigma}(X)} \text { eval }\left(t_{1}\right) \oplus\left(t_{1}, t_{2}\right)+\sum_{t_{1}, t_{2} \in T_{\Sigma}(X)} \operatorname{eval}\left(t_{2}\right) \oplus\left(t_{1}, t_{2}\right)+ \\
& \sum_{t_{1}, t_{2} \in T_{\Sigma}(X)} \operatorname{eval}\left(t_{1}\right) \text { eval }\left(t_{2}\right) \otimes\left(t_{1}, t_{2}\right)+\sum_{t \in T_{\Sigma}(X)}-\operatorname{eval}(t) \ominus t+ \\
& \sum_{x \in \Sigma} \operatorname{eval}(x) x= \\
& \sum_{t_{1}, t_{2} \in T_{\Sigma}(X)} \operatorname{eval}\left(\oplus\left(t_{1}, t_{2}\right)\right) \oplus\left(t_{1}, t_{2}\right)+\sum_{t_{1}, t_{2} \in T_{\Sigma}(X)} \operatorname{eval}\left(\otimes\left(t_{1}, t_{2}\right)\right) \otimes\left(t_{1}, t_{2}\right)+ \\
& \sum_{t \in T_{\Sigma}(X)} \text { eval }(\ominus(t)) \ominus t+\sum_{x \in \Sigma} \operatorname{eval}(x) x= \\
& \sum_{t \in T_{\Sigma}(X)} \operatorname{eval}(t) t=\text { eval, } \\
& \oplus(\operatorname{char}, \operatorname{char})+\otimes(\text { char, char })+\ominus(\operatorname{char})+\sum_{x \in \Sigma} x= \\
& \sum_{t_{1}, t_{2} \in T_{\Sigma}(X)} \oplus\left(t_{1}, t_{2}\right)+ \\
& \sum_{t_{1}, t_{2} \in T_{\Sigma}(X)} \otimes\left(t_{1}, t_{2}\right)+\sum_{t \in T_{\Sigma}(X)} \ominus(t)+\sum_{x \in \Sigma} x=\operatorname{char} .
\end{aligned}
$$

Consider now the finite tree automaton $\mathfrak{A}=(Q, M, S, P)$, where $Q=$ $\left\{z_{1}, z_{2}\right\}, S_{z_{1}}=y_{1}, S_{z_{2}}=0, P_{z_{1}}=\sum_{x \in \Sigma} \operatorname{eval}(x) x, P_{z_{2}}=\sum_{x \in \Sigma} x$, and the nonnull entries of $M$ are given by

$$
\begin{array}{ll}
M_{z_{1},\left(z_{1}, z_{1}\right)}=(-1) \ominus\left(y_{1}\right), & M_{z_{2},\left(z_{2}, z_{2}\right)}=\ominus\left(y_{1}\right), \\
M_{z_{1},\left(z_{1}, z_{1}\right)}=\otimes\left(y_{1}, y_{2}\right), & M_{z_{1},\left(z_{1}, z_{2}\right)}=\oplus\left(y_{1}, y_{2}\right), \\
M_{z_{1},\left(z_{2}, z_{1}\right)}=\oplus\left(y_{1}, y_{2}\right), & M_{z_{2},\left(z_{2}, z_{2}\right)}=\oplus\left(y_{1}, y_{2}\right)+\otimes\left(y_{1}, y_{2}\right) .
\end{array}
$$

Then we obtain $\|\mathfrak{A}\|=\sigma_{1}=$ eval.

Let $X=\{a, b, c\}$ and $t=\oplus(\ominus a, \otimes(b, c))$. Then there are two computations for $t$ starting at $z_{1}$ and none starting from $z_{2}$. These two computations are given in the following pictorial form:

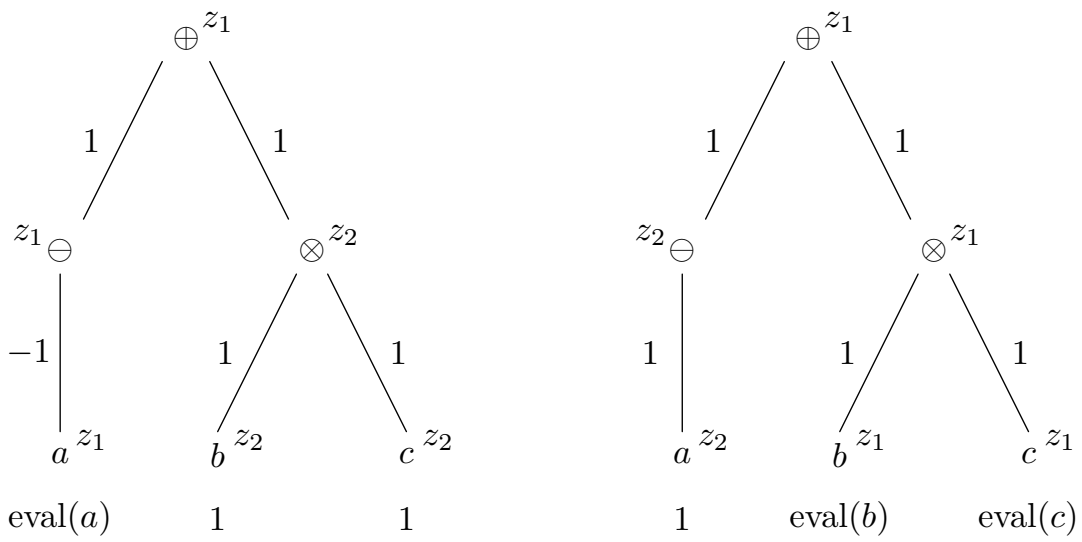

Hence, $(\|\mathfrak{A}\|, t)=-\operatorname{eval}(a)+\operatorname{eval}(b) \operatorname{eval}(c)$.

Example 3.1 gives also an intuitive feeling, how a finite nondeterministic root-to-frontier tree recognizer is simulated by a finite tree automaton over the 
semiring $\mathbb{B}$. Formally, we have the following theorem that is a slight adaption of Theorem 3.6 of Kuich [38].

Theorem 3.7 For each finite nondeterministic root-to-frontier tree recognizer A in the sense of Gécseg, Steinby [24] there exists a simple proper finite polynomial tree automaton $\mathfrak{A}$ over the Boolean semiring $\mathbb{B}$ auch that $\|\mathfrak{A}\|=T(\mathbf{A})$, and vice versa.

\section{Closure properties and a Kleene Theorem for recognizable tree series}

In this section we prove a Kleene Theorem for recognizable tree series. (See Thatcher, Wright [58], Bozapalidis [10], Gruska [30], Gécseg, Steinby [24, 25], Kuich [37].) This Kleene Theorem allows the definition of recognizable tree series by expressions which are analogous to regular expressions.

We first show that $A^{\text {rec }}\left\langle\left\langle T_{\Sigma}(X)\right\rangle\right\rangle$ is a distributive $\Sigma$-algebra.

Theorem 4.1 $\left\langle A^{\mathrm{rec}}\left\langle\left\langle T_{\Sigma}(X)\right\rangle\right\rangle,+, \cup, \bar{\Sigma}\right\rangle$ is a distributive $\Sigma$-algebra closed under scalar product that contains $A\left\langle T_{\Sigma}(X)\right\rangle$.

Proof. Let $s_{j} \in A^{\text {rec }}\left\langle\left\langle T_{\Sigma}(X)\right\rangle\right\rangle, 1 \leq j \leq m$, where $m \geq 2$ is greater or equal to the maximal rank of an operation symbol in $\Sigma$, be the first components of the unique solution of the simple proper finite polynomial systems (written in matrix notation) $z^{j}=p^{j}\left(z^{j}\right), 1 \leq j \leq m$, with mutually disjoint variable alphabets. Let $\sigma^{j}$ be the unique solution of $z^{j}=p^{j}\left(z^{j}\right), 1 \leq j \leq m$, with $\sigma_{1}^{j}=s_{j}$.

(i) Consider the system

$$
z_{0}=p_{1}^{1}\left(z^{1}\right)+p_{1}^{2}\left(z^{2}\right), \quad z^{1}=p^{1}\left(z^{1}\right), \quad z^{2}=p^{2}\left(z^{2}\right) .
$$

It is again simple and proper. We claim that its unique solution is given by $\left(s_{1}+s_{2}, \sigma^{1}, \sigma^{2}\right)$ and show it by substitution:

$$
p_{1}^{1}\left(\sigma^{1}\right)+p_{1}^{2}\left(\sigma^{2}\right)=\sigma_{1}^{1}+\sigma_{1}^{2}=s_{1}+s_{2}, \quad p^{j}\left(\sigma^{j}\right)=\sigma^{j}, \quad j=1,2 .
$$

(ii) Let $\omega \in \Sigma_{k}, k \geq 0$, and consider the system

$$
z_{0}=\omega\left(z_{1}^{1}, \ldots, z_{1}^{k}\right), \quad z^{j}=p^{j}\left(z^{j}\right), \quad 1 \leq j \leq k .
$$

It is again simple and proper. We claim that its unique solution is given by $\left(\bar{\omega}\left(s_{1}, \ldots, s_{k}\right), \sigma^{1}, \ldots, \sigma^{k}\right)$ and show it by substitution:

$$
\bar{\omega}\left(\sigma_{1}^{1}, \ldots, \sigma_{1}^{k}\right)=\bar{\omega}\left(s_{1}, \ldots, s_{k}\right), \quad p^{j}\left(\sigma^{j}\right)=\sigma^{j}, \quad 1 \leq j \leq k .
$$

(iii) Let $a \in A$ and consider the system

$$
z_{0}=a p_{1}^{1}\left(z^{1}\right), \quad z^{1}=p^{1}\left(z^{1}\right) .
$$


It is again simple and proper. We claim that its unique solution is given by $\left(a s_{1}, \sigma^{1}\right)$ and show it by substitution:

$$
a p_{1}^{1}\left(\sigma^{1}\right)=a s_{1}, \quad p^{1}\left(\sigma^{1}\right)=\sigma^{1} .
$$

(iv) For $s \in A\left\langle T_{\Sigma}(X)\right\rangle, s$ is the unique solution of the system $z_{0}=s$.

In the sequel, $Z=\left\{z_{j} \mid j \geq 1\right\}, Z_{n}=\left\{z_{j} \mid 1 \leq j \leq n\right\}, Z_{0}=\emptyset$. We introduce the following notation: Let $s \in A\left\langle\left\langle T_{\Sigma}\left(X \cup Z_{n}\right)\right\rangle\right\rangle$. Then we denote the least $\sigma \in$ $A\left\langle\left\langle T_{\Sigma}\left(X \cup\left\{z_{1}, \ldots, z_{i-1}, z_{i+1}, \ldots, z_{n}\right\}\right)\right\rangle\right\rangle$ such that $s\left(z_{1}, \ldots, z_{i-1}, \sigma, z_{i+1}, \ldots, z_{n}\right)=$ $\sigma$ by $\mu z_{i} . s\left(z_{1}, \ldots, z_{n}\right), 1 \leq i \leq n$. This means that $\mu z_{i} . s$ is the least fixed point solution of the system $z_{i}=s\left(z_{1}, \ldots, z_{i}, \ldots, z_{n}\right)$; this system consists of one equation only and its single variable is $z_{i}$.

A distributive $\Sigma$-algebra $\langle V,+, 0, \bar{\Sigma}\rangle, V \subseteq A\left\langle\left\langle T_{\Sigma}(X \cup Z)\right\rangle\right\rangle$ is called rationally closed iff $V$ is closed under scalar product and for all $s \in V$ and $z \in Z$ the formal tree series $\mu z . s$ is again in $V$. By definition, $A^{\text {rat }}\left\langle\left\langle T_{\Sigma}(X \cup Z)\right\rangle\right\rangle$ is the least rationally closed distributive $\Sigma$-algebra containing $A\left\langle T_{\Sigma}(X \cup Z)\right\rangle$. Observe that for each $s \in A^{\text {rat }}\left\langle\left\langle T_{\Sigma}(X \cup Z)\right\rangle\right\rangle$ there exists an $m \geq 0$ such that $\operatorname{supp}(s) \subseteq$ $T_{\Sigma}\left(X \cup Z_{m}\right)$.

We will prove that $A^{\text {rat }}\left\langle\left\langle T_{\Sigma}(X \cup Z)\right\rangle\right\rangle=A^{\text {rec }}\left\langle\left\langle T_{\Sigma}(X \cup Z)\right\rangle\right\rangle$. Before proving the main result of this section we apply a few results of the fixed point theory of continuous functions to systems.

(1) The parameter identity. Let $r \in A\left\langle\left\langle T_{\Sigma}(X \cup Y)\right\rangle\right\rangle$ and denote $r^{\prime}=\mu y . r$, $y \in Y$. Let $y_{i} \neq y$ and $\tau_{i} \in A\left\langle\left\langle T_{\Sigma}(X \cup(Y-\{y\}))\right\rangle\right\rangle, 1 \leq i \leq n$. Then $r^{\prime}\left[\tau_{1} / y_{1}, \ldots, \tau_{n} / y_{n}\right]=\mu y \cdot\left(r\left[\tau_{1} / y_{1}, \ldots, \tau_{n} / y_{n}\right]\right)$.

(2) The Bekić-De Bakker-Scott rule. Consider the system $y_{i}=r_{i}, 1 \leq i \leq$ $n, r_{i} \in A\left\langle\left\langle T_{\Sigma}(X \cup Y)\right\rangle\right\rangle$ with variables $y_{1}, \ldots, y_{n}$ and $m \in\{1, \ldots, n\}$. Let $\left(\sigma_{m+1}, \ldots, \sigma_{n}\right)$ be the least solution of the system $y_{i}=r_{i}, m+1 \leq i \leq$ $n$. Furthermore, let $\left(\tau_{1}, \ldots, \tau_{m}\right)$ be the least solution of the system $y_{i}=$ $r_{i}\left[\sigma_{m+1} / y_{m+1}, \ldots, \sigma_{n} / y_{n}\right], 1 \leq i \leq m$. Then

$$
\left(\tau_{1}, \ldots, \tau_{m}, \sigma_{m+1}\left[\tau_{1} / y_{1}, \ldots, \tau_{m} / y_{m}\right], \ldots, \sigma_{n}\left[\tau_{1} / y_{1}, \ldots, \tau_{m} / y_{m}\right]\right)
$$

is the least solution of the original system $y_{i}=r_{i}, 1 \leq i \leq n$.

We first show that $A^{\text {rat }}\left\langle\left\langle T_{\Sigma}(X \cup Z)\right\rangle\right\rangle$ is closed under substitution.

Theorem 4.2 Assume that $s\left(z_{1}, \ldots, z_{n}\right)$ and $\sigma_{j}, 1 \leq j \leq n$, are in $A^{\text {rat }}\left\langle\left\langle T_{\Sigma}(X \cup\right.\right.$ $Z)\rangle\rangle$. Then $s\left(\sigma_{1}, \ldots, \sigma_{n}\right)$ is again in $A^{\text {rat }}\left\langle\left\langle T_{\Sigma}(X \cup Z)\right\rangle\right\rangle$.

Proof. The proof is by induction on the number of applications of the operations $\bar{\omega} \in \bar{\Sigma},+$, scalar product and $\mu$ to generate $s\left(z_{1}, \ldots, z_{n}\right)$ from polynomials.

(i) Let $s\left(z_{1}, \ldots, z_{n}\right) \in A\left\langle T_{\Sigma}(X \cup Z)\right\rangle$. Since $s\left(\sigma_{1}, \ldots, \sigma_{n}\right)$ is generated from $\sigma_{1}, \ldots, \sigma_{n}$ by application of sum, $\bar{\omega} \in \bar{\Sigma}$ and scalar product, we infer that $s\left(\sigma_{1}, \ldots, \sigma_{n}\right) \in A^{\text {rat }}\left\langle\left\langle T_{\Sigma}(X \cup Z)\right\rangle\right.$.

(ii) We only prove the case of the operator $\mu$. Assume that, for $1 \leq j \leq n$, $\operatorname{supp}(s) \subseteq T_{\Sigma}\left(X \cup Z_{m}\right)$ for some $m \geq 0$. Choose a $z=z_{k} \in Z$ with $k>m$. 
Without loss of generality assume that $s\left(z_{1}, \ldots, z_{n}\right)=\mu z \cdot r\left(z_{1}, \ldots, z_{n}, z\right)$ (the variable $z$ is "bound"), where $r\left(z_{1}, \ldots, z_{n}, z\right) \in A^{\text {rat }}\left\langle\left\langle T_{\Sigma}(X \cup Z)\right\rangle\right.$. By induction hypothesis, we have $r\left(\sigma_{1}, \ldots, \sigma_{n}, z\right) \in A^{\text {rat }}\left\langle\left\langle T_{\Sigma}(X \cup Z)\right\rangle\right.$. Hence, $s\left(\sigma_{1}, \ldots, \sigma_{n}\right)=$ $\mu z . r\left(\sigma_{1}, \ldots, \sigma_{n}, z\right) \in A^{\text {rat }}\left\langle\left\langle T_{\Sigma}(X \cup Z)\right\rangle\right\rangle$ by the parameter identity.

Theorem 4.3 (Bozapalidis [10], Section 5.) $A^{\mathrm{rat}}\left\langle\left\langle T_{\Sigma}(X \cup Z)\right\rangle\right\rangle=A^{\mathrm{rec}}\left\langle\left\langle T_{\Sigma}(X \cup\right.\right.$ $Z)\rangle$.

Proof. (i) We show $A^{\mathrm{rec}}\left\langle\left\langle T_{\Sigma}(X \cup Z)\right\rangle\right\rangle \subseteq A^{\mathrm{rat}}\left\langle\left\langle T_{\Sigma}(X \cup Z)\right\rangle\right\rangle$. The proof is by induction on the number of variables of finite polynomial systems. We use the following induction hypothesis: If $\tau=\left(\tau_{1}, \ldots, \tau_{n}\right), \tau_{i} \in A^{\mathrm{rec}}\left\langle\left\langle T_{\Sigma}(X \cup Z)\right\rangle\right.$, $1 \leq i \leq n$, is the least solution of a finite polynomial system $z_{i}=q_{i}\left(z_{1}, \ldots, z_{n}\right)$, $1 \leq i \leq n$, with $n$ variables $z_{1}, \ldots, z_{n}$, where $q_{i}\left(z_{1}, \ldots, z_{n}\right) \in A\left\langle T_{\Sigma}(X \cup Z)\right\rangle$ then $\tau_{i} \in A^{\text {rat }}\left\langle\left\langle T_{\Sigma}(X \cup Z)\right\rangle\right\rangle$.

(1) Let $n=1$ and assume that $s \in A^{\text {rec }}\left\langle\left\langle T_{\Sigma}(X \cup Z)\right\rangle\right\rangle$ is the least solution of the finite polynomial system $z_{1}=p\left(z_{1}\right)$. Since $p\left(z_{1}\right)$ is a polynomial, $s=$ $\mu z_{1} . p\left(z_{1}\right) \in A^{\text {rat }}\left\langle\left\langle T_{\Sigma}(X \cup Z)\right\rangle\right.$.

(2) Consider the finite polynomial system $z_{i}=q_{i}\left(z_{1}, \ldots, z_{n+1}\right), 1 \leq i \leq n+1$, $n \geq 1$. Let $\tau\left(z_{1}\right)=\left(\tau_{2}\left(z_{1}\right), \ldots, \tau_{n+1}\left(z_{1}\right)\right), \tau_{i}\left(z_{1}\right) \in A^{\text {rec }}\left\langle\left\langle T_{\Sigma}(X \cup Z)\right\rangle\right\rangle, 2 \leq i \leq$ $n+1$, be the least solution of the finite polynomial system $z_{i}=q_{i}\left(z_{1}, \ldots, z_{n+1}\right)$, $2 \leq i \leq n+1$. By our induction hypothesis we infer that $\tau_{i}\left(z_{1}\right) \in A^{\text {rat }}\left\langle\left\langle T_{\Sigma}(X \cup\right.\right.$ $Z)\rangle\rangle$. Since $q_{1}\left(z_{1}, \ldots, z_{n+1}\right)$ is a polynomial, it is in $A^{\text {rat }}\left\langle\left\langle T_{\Sigma}(X \cup Z)\right\rangle\right\rangle$. Hence, by Theorem $4.2, p\left(z_{1}\right)=q_{1}\left(z_{1}, \tau_{2}\left(z_{1}\right), \ldots, \tau_{n+1}\left(z_{1}\right)\right)$ is in $A^{\text {rat }}\left\langle\left\langle T_{\Sigma}(X \cup Z)\right\rangle\right\rangle$. This implies that $\mu z_{1} . p\left(z_{1}\right)$ is in $A^{\text {rat }}\left\langle\left\langle T_{\Sigma}(X \cup Z)\right\rangle\right\rangle$. Again by Theorem 4.2, $\tau_{i}\left(\mu z_{1} . p\left(z_{1}\right)\right) \in A^{\text {rat }}\left\langle\left\langle T_{\Sigma}(X \cup Z)\right\rangle\right\rangle, 2 \leq i \leq n+1$. By the Bekić-De BakkerScott rule, $\left(\mu z_{1} \cdot p\left(z_{1}\right), \tau_{2}\left(\mu z_{1} \cdot p\left(z_{1}\right)\right), \ldots, \tau_{n+1}\left(\mu z_{1} \cdot p\left(z_{1}\right)\right)\right)$ is the least solution of the finite polynomial system $z_{i}=q_{i}\left(z_{1}, \ldots, z_{n+1}\right), 1 \leq i \leq n+1$. Hence, the components of the least solution of $z_{i}=q_{i}\left(z_{1}, \ldots, z_{n+1}\right), 1 \leq i \leq n+1$, are in $A^{\text {rat }}\left\langle\left\langle T_{\Sigma}(X \cup Z)\right\rangle\right\rangle$.

(ii) We show that $A^{\text {rec }}\left\langle\left\langle T_{\Sigma}(X \cup Z)\right\rangle\right\rangle$ is a rationally closed distributive $\Sigma$ algebra that contains $A\left\langle T_{\Sigma}(X \cup Z)\right\rangle$. This will imply $A^{\text {rat }}\left\langle\left\langle T_{\Sigma}(X \cup Z)\right\rangle\right\rangle \subseteq$ $A^{\text {rec }}\left\langle\left\langle T_{\Sigma}(X \cup Z)\right\rangle\right\rangle$. By Theorem 4.1 (with $X \cup Z$ instead of $\left.X\right), A^{\text {rec }}\left\langle\left\langle T_{\Sigma}(X \cup Z)\right\rangle\right\rangle$ is a distributive $\Sigma$-algebra closed under scalar product that contains $A\left\langle T_{\Sigma}(X \cup\right.$ $Z)\rangle$. Hence, we have only to show that $\mu z . s, s \in A^{\mathrm{rec}}\left\langle\left\langle T_{\Sigma}(X \cup Z)\right\rangle\right\rangle$, is in $A^{\text {rec }}\left\langle\left\langle T_{\Sigma}(X \cup Z)\right\rangle\right\rangle$. Let $\left(\tau_{2}\left(z_{1}\right), \ldots, \tau_{n+1}\left(z_{1}\right)\right)$ be the least solution of the finite polynomial system $z_{i}=p_{i}\left(z_{1}, \ldots, z_{n+1}\right), 2 \leq i \leq n+1$, and take $s=\tau_{2}\left(z_{1}\right)$. Consider now the finite polynomial system $z_{1}=p_{2}\left(z_{1}\right), z_{i}=p_{i}\left(z_{1}, \ldots, z_{n+1}\right)$, $2 \leq i \leq n+1$. Then, by the Bekić-De Bakker-Scott rule, $\mu z_{1} \cdot \tau_{2}\left(z_{1}\right)$ is the first component of its least solution.

Analogous to the regular expressions (see Salomaa [54]) and to the $\Sigma Z$ expressions (see Gécseg, Steinby [25]) we define now recognizable tree series expressions. Assume that $A, \Sigma, X, Z$ and $U=\{+, \cdot, \mu,[]$,$\} are mutually disjoint.$ A word $E$ over $A \cup \Sigma \cup X \cup Z \cup U$ is a recognizable tree series expression over $(A, \Sigma, X, Z)$ iff 
(i) $E$ is a symbol of $X \cup Z$, or

(ii) $E$ is of one of the forms $\left[E_{1}+E_{2}\right], \omega\left(E_{1}, \ldots, E_{k}\right), a E_{1}$, or $\mu z . E_{1}$, where $E_{1}, E_{2}, \ldots, E_{k}$ are recognizable tree series expressions over $(A, \Sigma, X, Z)$, $\omega \in \Sigma_{k}, k \geq 0, a \in A$, and $z \in Z$.

Each recognizable tree series expression $E$ over $(A, \Sigma, X, Z)$ denotes a formal tree series $|E|$ in $A\left\langle\left\langle T_{\Sigma}(X \cup Z)\right\rangle\right\rangle$ according to the following conventions:

(i) If $E$ is in $X \cup Z$ then $E$ denotes the tree series $E$, i. e., $|E|=E$.

(ii) For recognizable tree series expressions $E_{1}, \ldots, E_{k}$ over $(A, \Sigma, X, Z), \omega \in$ $\Sigma_{k}, k \geq 0, a \in A, z \in Z$, we define

$$
\begin{aligned}
& \left|\left[E_{1}+E_{2}\right]\right|=\left|E_{1}\right|+\left|E_{2}\right|, \\
& \left|\omega\left(E_{1}, \ldots, E_{k}\right)\right|=\bar{\omega}\left(\left|E_{1}\right|, \ldots,\left|E_{k}\right|\right), \\
& \left|a E_{1}\right|=a\left|E_{1}\right| \\
& \left|\mu z . E_{1}\right|=\mu z .\left|E_{1}\right| .
\end{aligned}
$$

Let $\Phi_{1}$ and $\Phi_{2}$ be mappings from the set of recognizable tree series expressions over $(A, \Sigma, X, Z)$ into the set of finite subsets of $X \cup Z$ defined by

(i) $\Phi_{1}(x)=\emptyset, \Phi_{2}(x)=\{x\}, x \in X$,

$$
\Phi_{1}(z)=\{z\}, \Phi_{2}(z)=\emptyset, z \in Z \text {. }
$$

(ii) $\Phi_{j}\left(\left[E_{1}+E_{2}\right]\right)=\Phi_{j}\left(E_{1}\right) \cup \Phi_{j}\left(E_{2}\right)$,

$$
\begin{aligned}
& \Phi_{j}\left(\omega\left(E_{1}, \ldots, E_{k}\right)\right)=\Phi_{j}\left(E_{1}\right) \cup \ldots \cup \Phi_{j}\left(E_{k}\right), \\
& \Phi_{j}\left(a E_{1}\right)=\Phi_{j}\left(E_{1}\right),
\end{aligned}
$$$$
\Phi_{j}\left(\mu z \cdot E_{1}\right)=\Phi_{j}\left(E_{1}\right)-\{z\}, j=1,2
$$

for recognizable tree series expressions $E_{1}, E_{2}, \ldots, E_{k}$ over $(A, \Sigma, X, Z)$, $\omega \in \Sigma_{k}, k \geq 0, a \in A$, and $z \in Z$.

Given a recognizable tree series expression $E$ over $(A, \Sigma, X, Z), \Phi_{1}(E) \subseteq Z$ contains the "free variables" of $E$, while $\Phi_{2}(E) \subseteq X$ contains the used symbols of the leaf alphabet $X$. This means that $|E|$ is a formal tree series in $A\left\langle\left\langle T_{\Sigma}\left(\Phi_{2}(E) \cup\right.\right.\right.$ $\left.\left.\left.\Phi_{1}(E)\right)\right\rangle\right\rangle$. Theorem 4.3 and the above definitions yield some corollaries.

Corollary $4.4 A$ tree series $s$ is in $A^{\mathrm{rat}}\left\langle\left\langle T_{\Sigma}(X \cup Z)\right\rangle \cap A\left\langle\left\langle T_{\Sigma}\left(\Phi_{2}(E) \cup \Phi_{1}(E)\right)\right\rangle\right\rangle\right.$ iff there exists a recognizable tree series expression $E$ over $(A, \Sigma, X, Z)$ such that $s=|E|$.

Corollary 4.5 A tree series $s$ is in $A^{\text {rat }}\left\langle\left\langle T_{\Sigma}(X \cup Z)\right\rangle \cap \cap A\left\langle\left\langle T_{\Sigma}\left(\Phi_{2}(E)\right)\right\rangle\right\rangle\right.$ iff there exists a recognizable tree series expression $E$ over $(A, \Sigma, X, Z)$ such that $s=|E|$, where $\Phi_{1}(E)=\emptyset$.

Corollary 4.6 $A$ tree series $s$ is in $A^{\text {rec }}\left\langle\left\langle T_{\Sigma}\left(\Phi_{2}(E)\right)\right\rangle\right\rangle$ iff there exists a recognizable tree series expression $E$ over $(A, \Sigma, X, Z)$ such that $s=|E|$, where $\Phi_{1}(E)=\emptyset$. 
Observe that our Corollary 4.4 is stronger than "Kleene's Theorem" of Bozapalidis [10], Section 5, since we can use our Theorem 4.2 and do not need "closure under substitution" in the definition of an rationally closed distributive ¿-algebra.

We summarize our results in a Kleene-like theorem (see Bozapalidis [10]).

Theorem 4.7 The following statements on a power series $r \in A\left\langle\left\langle T_{\Sigma}(X)\right\rangle\right\rangle$ are equivalent:

(i) $r$ is a component of the least solution of a finite polynomial system;

(ii) $r$ is the behavior of a finite polynomial tree series automaton;

(iii) there exists a recognizable tree series expression $E$ over $(A, \Sigma, X, Z)$, where $\phi_{1}(E)=\emptyset$, such that $r=|E|$.

Proof. By Corollary 3.6 and Theorem 4.3.

In the characterization of the recognizable tree languages, Gécseg, Steinby [25] use the following closure operation for a tree language $r\left(y_{1}, \ldots, y_{n}, y\right) \in$ $\mathbb{B}\left\langle\left\langle T_{\Sigma}\left(X \cup\left\{y_{1}, \ldots, y_{n}, y\right\}\right)\right\rangle\right\rangle, y=y_{n+1}$ :

$$
\begin{aligned}
r^{0, y}\left(y_{1}, \ldots, y_{n}, y\right) & =\{y\}, \\
r^{j+1, y}\left(y_{1}, \ldots, y_{n}, y\right) & =r\left(y_{1}, \ldots, y_{n}, r^{j, y}\left(y_{1}, \ldots, y_{n}, y\right)\right) \cup r^{j, y}\left(y_{1}, \ldots, y_{n}, y\right), j \geq 0, \\
r^{* y}\left(y_{1}, \ldots, y_{n}, y\right) & =\bigcup_{j \geq 0} r^{j, y}\left(y_{1}, \ldots, y_{n}, y\right) .
\end{aligned}
$$

(Here we use the isomorphism between $\mathfrak{P}\left(T_{\Sigma}(Y)\right.$ ) and $\mathbb{B}\left\langle\left\langle T_{\Sigma}(Y)\right\rangle\right\rangle$.) Consider now the finite polynomial system over $\mathbb{B}$ with just one variable $y_{0}$

$$
y_{0}=r\left(y_{1}, \ldots, y_{n}, y_{0}\right)+y \text {. }
$$

Using the equality

$$
r^{j+1, y}\left(y_{1}, \ldots, y_{n}, y\right)=r\left(y_{1}, \ldots, y_{n}, r^{j, y}\left(y_{1}, \ldots, y_{n}, y\right)\right)+y, \quad j \geq 0,
$$

an easy proof by induction on the elements of the approximation sequence shows that its least solution is given by $r^{* y}\left(y_{1}, \ldots, y_{n}, y\right)$. Hence, $\mu y_{0} \cdot\left(r\left(y_{1}, \ldots, y_{n}, y_{0}\right)+\right.$ $y)=r^{* y}\left(y_{1}, \ldots, y_{n}, y\right)$.

Bozapalidis [10] had the idea to replace $\mu y_{0} \cdot\left(r\left(y_{1}, \ldots, y_{n}, y_{0}\right)+y\right)$ by $\mu y . r\left(y_{1}, \ldots, y_{n}, y\right)$. (For context-free languages, Gruska [30] used implicitely this closure operator; see Kuich [37].) We have used this closure operator of Bozapalidis [10] in our paper. The essential difference of the two closure operators is that $r^{* y}\left(y_{1}, \ldots, y_{n}, y\right) \in \mathbb{B}\left\langle\left\langle T_{\Sigma}\left(X \cup Y_{n} \cup\{y\}\right)\right\rangle\right\rangle$, while $\mu y . r\left(y_{1}, \ldots, y_{n}, y\right) \in$ $\mathbb{B}\left\langle\left\langle T_{\Sigma}\left(X \cup Y_{n}\right)\right\rangle\right\rangle$.

By the parameter identity we can even say more:

$$
\mu y \cdot r\left(y_{1}, \ldots, y_{n}, y\right)=r^{* y}\left(y_{1}, \ldots, y_{n}, 0\right) \text {. }
$$


Hence, our interpretation of a recognizable tree series expression over $(\mathbb{B}, \Sigma, X, Z)$ that is given below is different from that by Gécseg, Steinby [25].

Each recognizable tree series expression $E$ over $(\mathbb{B}, \Sigma, X, Z)$ denotes a tree language $|E| \subseteq T_{\Sigma}(X \cup Z)$ according to the following conventions (expressions of the form $0 E_{1}, 1 E_{1}$ are not needed):

(i) The tree language denoted by $x \in X$ is $\{x\}$.

(ii) The tree language denoted by $z \in Z$ is $\{z\}$.

(iii) For recognizable tree series expressions $E_{1}, E_{2}, \ldots, E_{k}$ over $(\mathbb{B}, \Sigma, X, Z)$, $\omega \in \Sigma_{k}, k \geq 0$, and $z \in Z$,

$$
\begin{gathered}
\left|\left[E_{1}+E_{2}\right]\right|=\left|E_{1}\right| \cup\left|E_{2}\right|, \\
\left|\omega\left(E_{1}, \ldots, E_{k}\right)\right|=\bar{\omega}\left(\left|E_{1}\right|, \ldots,\left|E_{k}\right|\right), \\
\left|\mu z . E_{1}\right|=\mu z .\left|E_{1}\right| .
\end{gathered}
$$

In the next theorem we use the notation of Gécseg, Steinby [25].

Theorem 4.8 The following statements on a tree language $L \subseteq T_{\Sigma}(X)$ are equivalent:

(i) L is generated by a regular $\Sigma X$-grammar;

(ii) L is recognized by a nondeterministic finite root-to-frontier $\Sigma X$-recognizer;

(iii) $L=|E|$, where $E$ is a recognizable tree series expression over $(\mathbb{B}, \Sigma, X, Z)$ and $\phi_{1}(E)=\emptyset$.

Observe that Theorem 4.8 is stronger than Proposition 9.3 (Kleene's Theorem) of Gécseg, Steinby [25], since we do not need "closure under substitution" for our tree expressions over $(\mathbb{B}, \Sigma, X, Z)$.

Example 4.1. Let $\Sigma=\Sigma_{0} \cup \Sigma_{1} \cup \Sigma_{2}, \Sigma_{0}=\{c, d\}, \Sigma_{1}=\{g\}, \Sigma_{2}=\{f\}, z, z_{1} \in Z$ and consider the recognizable tree series expression $\left[g(c)+\mu z_{1} \cdot\left[f\left(c, z_{1}\right)+z\right]\right]$ over $(\mathbb{B}, \Sigma, X, Z)$. It denotes

$\left|\left[g(c)+\mu z_{1} \cdot\left[f\left(c, z_{1}\right)+z\right]\right]\right|=g(c)+z+f(c, z)+f(c, f(c, z))+f(c, f(c, f(c, z)))+\ldots$

Moreover,

$$
\left|\left[g(c)+\mu z_{1} \cdot\left[f\left(c, z_{1}\right)+d\right]\right]\right|=\left|\left[g(c)+\mu z_{1} \cdot\left[f\left(c, z_{1}\right)+z\right]\right]\right|\left[d / z_{1}\right] .
$$

Compare this with the second paragraph on page 21 of Gécseg, Steinby [24]. 


\section{Pushdown tree automata, algebraic tree sys- tems, and a Kleene Theorem}

In this section we consider pushdown tree automata and algebraic tree systems. Moreover, we prove a Kleene Theorem due to Bozapalidis [11].

Guessarian [32] introduced the notion of a (top-down) pushdown tree automaton and showed that these pushdown tree automata recognize exactly the class of context-free tree languages. Here a tree language is called context-free iff it is generated by a context-free tree grammar. Moreover, she showed that pushdown tree automata are equivalent to restricted pushdown tree automata, i. e., to pushdown automata, whose pushdown store is linear.

Kuich [43] generalized these results of Guessarian [32] to formal tree series. He defined pushdown tree automata whose behaviors are formal tree series and showed that the class of behaviors of these pushdown tree automata coincides with the class of algebraic tree series. Here a tree series is called algebraic iff it is the initial component of the least solution of an algebraic tree system with initial function variable. These algebraic tree systems are a generalization of the context-free tree grammars (see Rounds [51] and Gécseg, Steinby [25]). They are a particular instance of the second-order systems of Bozapalidis [11]. The presentation follows Kuich [43].

A pushdown tree automaton (with input alphabet $\Sigma$ and leaf alphabet $X$ ) over the semiring $A$

$$
\mathfrak{P}=\left(Q, \Gamma, Z, Y, M, S, p_{0}, P\right)
$$

is given by

(i) a finite nonempty set $Q$ of states;

(ii) a finite ranked alphabet $\Gamma=\Gamma_{0} \cup \Gamma_{1} \cup \ldots \cup \Gamma_{\bar{m}}$ of pushdown symbols;

(iii) a finite alphabet $Z=\left\{z_{1}, \ldots, z_{\bar{m}}\right\}$ of pushdown variables;

(iv) a finite alphabet $Y=\left\{y_{1}, \ldots, y_{k}\right\}$ of variables;

(v) a pushdown tree transition matrix $M$ of order $k$;

(vi) $S \in\left(A\left\langle T_{\Sigma}\left(X \cup Y_{1}\right)\right\rangle\right)^{1 \times Q}$, called the initial state vector;

(vii) $p_{0} \in \Gamma_{0}$, called the initial pushdown symbol;

(viii) a finite family $P=\left(P_{g\left(z_{1}, \ldots, z_{m}\right)} \mid g \in \Gamma_{m}, 0 \leq m \leq \bar{m}\right)$ of final state

$$
\text { vectors } P_{g\left(z_{1}, \ldots, z_{m}\right)} \in\left(A\left\langle T_{\Sigma}(X)\right\rangle\right)^{Q \times 1}, g \in \Gamma_{m}, 0 \leq m \leq \bar{m} \text {. }
$$

Here a pushdown tree transition matrix of order $k$ is a matrix

$$
M \in\left(\left(A\left\langle T_{\Sigma}\left(X \cup Y_{k}\right)\right\rangle\right)^{Q \times Q^{k}}\right)^{T_{\Gamma}(Z) \times T_{\Gamma}(Z)^{k}}
$$

which satisfies the following two conditions: 
(i) for all $t, t_{1}, \ldots, t_{k} \in T_{\Gamma}(Z)$

$$
M_{t,\left(t_{1}, \ldots, t_{k}\right)}=\left\{\begin{array}{c}
\sum M_{g\left(z_{1}, \ldots, z_{m}\right),\left(v_{1}\left(z_{1}, \ldots, z_{m}\right), \ldots, v_{k}\left(z_{1}, \ldots, z_{m}\right)\right)} \\
\quad \text { where the sum extends over all } v_{1}, \ldots, v_{k} \in T_{\Gamma}\left(Z_{m}\right), \\
\quad \text { such that } t_{j}=v_{j}\left(u_{1}, \ldots, u_{m}\right), 1 \leq j \leq k, \\
\text { if } t=g\left(u_{1}, \ldots, u_{m}\right), g \in \Gamma_{m}, u_{1}, \ldots, u_{m} \in T_{\Gamma}\left(Z_{m}\right) \\
0, \quad \text { otherwise. }
\end{array}\right.
$$

(ii) $M$ is row finite, i. e., for each $g \in \Gamma_{m}, 0 \leq m \leq \bar{m}$, there exists only finitely many blocks $M_{g\left(z_{1}, \ldots, z_{m}\right),\left(v_{1}, \ldots, v_{k}\right)}$, where $v_{1}, \ldots, v_{k} \in T_{\Gamma}\left(Z_{m}\right)$, that are unequal to zero;

Observe that if the root of $t$ is labeled by $g \in \Gamma_{m}$, then $M_{t,\left(t_{1}, \ldots, t_{k}\right)} \neq 0$ implies $t, t_{1}, \ldots, t_{k} \in T_{\Gamma}\left(Z_{m}\right)$.

Intuitively, the definition of the pushdown tree transition matrix means that the action of the pushdown tree automaton with tree $t=g\left(u_{1}, \ldots, u_{m}\right)$ on its pushdown tape depends only on the label $g$ of the root of $t$. Observe that a pushdown tree transition matrix of order $k$ is defined by its finitely many nonnull blocks of the form $M_{g\left(z_{1}, \ldots, z_{m}\right),\left(v_{1}, \ldots, v_{k}\right)}, g \in \Gamma_{m}$.

Let now $Z_{Q}=\left\{\left(z_{i}\right)_{q} \mid 1 \leq i \leq \bar{m}, q \in Q\right\}$ be an alphabet of variables and denote $Z_{Q}^{m}=\left\{\left(z_{i}\right)_{q} \mid 1 \leq i \leq m, q \in Q\right\}, 1 \leq m \leq \bar{m}, Z_{Q}^{0}=\emptyset$. Define $F \in\left(\left(A\left\langle T_{\Sigma}\left(X \cup Z_{Q}\right)\right\rangle\right)^{Q \times 1}\right)^{T_{\Gamma}(Z) \times 1}$ by its entries as follows:

(i) $\left(F_{t}\right)_{q}=\left(P_{g\left(z_{1}, \ldots, z_{m}\right)}\right)_{q}$ if $t=g\left(u_{1}, \ldots, u_{m}\right), g \in \Gamma_{m}, 0 \leq m \leq \bar{m}$, $u_{1}, \ldots, u_{m} \in T_{\Gamma}\left(Z_{m}\right), q \in Q$;

(ii) $\left(F_{z_{i}}\right)_{q}=\left(z_{i}\right)_{q}, 1 \leq i \leq \bar{m}, q \in Q$;

(iii) $\left(F_{t}\right)_{q}=0$, otherwise.

Hence, $F_{z_{i}}, 1 \leq i \leq \bar{m}$, is a column vector of dimension $Q$ whose $q$-entry, $q \in Q$, is the variable $\left(z_{i}\right)_{q}$.

The approximation sequence $\left(\tau^{j} \mid j \in \mathbb{N}\right), \tau^{j} \in\left(\left(A\left\langle T_{\Sigma}\left(X \cup Z_{Q}\right)\right\rangle\right)^{Q \times 1}\right)^{T_{\Gamma}(Z) \times 1}$, $j \geq 0$, associated with $\mathfrak{P}$ is defined as follows:

$$
\tau^{0}=0, \quad \tau^{j+1}=M\left(\tau^{j}, \ldots, \tau^{j}\right)+F, \quad j \geq 0 .
$$

This means that, for all $t \in T_{\Gamma}(Z)$, the block vectors $\tau_{t}^{j}$ of $\tau^{j}$ are defined by

$$
\tau_{t}^{0}=0, \quad \tau_{t}^{j+1}=\sum_{t_{1}, \ldots, t_{k} \in T_{\Gamma}(Z)} M_{t,\left(t_{1}, \ldots, t_{k}\right)}\left(\tau_{t_{1}}^{j}, \ldots, \tau_{t_{k}}^{j}\right)+F_{t}, j \geq 0 .
$$

Moreover, for all $t \in T_{\Gamma}(Z), q \in Q$,

$$
\begin{aligned}
& \left(\tau_{t}^{0}\right)_{q}=0, \\
& \left(\tau_{t}^{j+1}\right)_{q}=\sum_{t_{1}, \ldots, t_{k} \in T_{\Gamma}(Z)} \sum_{q_{1}, \ldots, q_{k} \in Q} \\
& \quad\left(M_{t,\left(t_{1}, \ldots, t_{k}\right)}\right)_{q,\left(q_{1}, \ldots, q_{k}\right)}\left(\left(\tau_{t_{1}}^{j}\right)_{q_{1}}, \ldots,\left(\tau_{t_{k}}^{j}\right)_{q_{k}}\right)+\left(F_{t}\right)_{q} j \geq 0 .
\end{aligned}
$$


Hence, for all $g \in \Gamma_{m}, 0 \leq m \leq \bar{m}$, and all $u_{1}, \ldots, u_{m} \in T_{\Gamma}\left(Z_{m}\right)$, we obtain, for all $j \geq 0$,

$$
\begin{aligned}
\tau_{g\left(u_{1}, \ldots, u_{m}\right)}^{j+1}= & \sum_{v_{1}, \ldots, v_{k} \in T_{\Gamma}\left(Z_{m}\right)} \\
& M_{g\left(z_{1}, \ldots, z_{m}\right),\left(v_{1}, \ldots, v_{k}\right)}\left(\tau_{v_{1}\left(u_{1}, \ldots, u_{m}\right)}^{j}, \ldots, \tau_{v_{k}\left(u_{1}, \ldots, u_{m}\right)}^{j}\right) \\
& +P_{g\left(z_{1}, \ldots, z_{m}\right)}
\end{aligned}
$$

and

$$
\tau_{z_{i}}^{j+1}=F_{z_{i}}, \quad z_{i} \in Z
$$

Let $\tau \in\left(\left(A\left\langle\left\langle T_{\Sigma}\left(X \cup Z_{Q}\right)\right\rangle\right\rangle\right)^{Q \times 1}\right)^{T_{\Gamma}(Z) \times 1}$ be the least upper bound of the approximation sequence associated with $\mathfrak{P}$. Then the behavior $\|\mathfrak{P}\|$ of the pushdown tree automaton $\mathfrak{P}$ is defined by

$$
\|\mathfrak{P}\|=S\left(\tau_{p_{0}}\right)=\sum_{q \in Q} S_{q}\left(\left(\tau_{p_{0}}\right)_{q}\right) .
$$

Observe that $\|\mathfrak{P}\|$ is a tree series in $A\left\langle\left\langle T_{\Sigma}(X)\right\rangle\right\rangle$. Furthermore, observe that $\left(\tau_{t}\right)_{q} \in A\left\langle\left\langle T_{\Sigma}\left(X \cup Z_{Q}\right)\right\rangle\right\rangle, t \in T_{\Gamma}(Z), q \in Q$, induces a mapping from $\left(A\left\langle\left\langle T_{\Sigma}(X \cup\right.\right.\right.$ $\left.\left.\left.\left.Z_{Q}\right)\right\rangle\right\rangle\right)^{\bar{m}|Q|}$ into $A\left\langle\left\langle T_{\Sigma}\left(X \cup Z_{Q}\right)\right\rangle\right\rangle$.

We now construct a polynomial tree automaton $\mathfrak{A}$ that is "isomorphic" to the pushdown tree automaton $\mathfrak{P}$. Let $\hat{M} \in\left(A\left\langle T_{\Sigma}\left(X \cup Y_{k}\right)\right\rangle\right)^{\left(T_{\Gamma}(Z) \times Q\right) \times\left(T_{\Gamma}(Z) \times Q\right)^{k}}$ and $\hat{F} \in\left(A\left\langle T_{\Sigma}\left(X \cup Z_{Q}\right)\right\rangle\right)^{\left(T_{\Gamma}(Z) \times Q\right) \times 1}$ be the isomorphic copies of $M$ and $F$, respectively. Observe that $\hat{M}$ is row finite. Furthermore define $\hat{S} \in\left(A\left\langle T_{\Sigma}(X \cup\right.\right.$ $\left.\left.\left.Y_{1}\right)\right\rangle\right)^{1 \times\left(T_{\Gamma}(Z) \times Q\right)}$ by $\hat{S}_{\left(p_{0}, q\right)}=S_{q}, \hat{S}_{(t, q)}=0, t \neq p_{0}, q \in Q$. Specify the polynomial tree automaton $\mathfrak{A}$ with input alphabet $\Sigma$ and leaf alphabet $X \cup Z_{Q}$ by

$$
\mathfrak{A}=\left(T_{\Gamma}(Z) \times Q, \hat{M}, \hat{S}, \hat{F}\right) .
$$

Then it is clear that $\|\mathfrak{A}\|=\|\mathfrak{P}\|$, i. e., our pushdown tree automaton fits into the general definition of a polynomial tree automaton. But for technical reasons, we prefer to work with the transition matrix $M$ in $\left(\left(A\left\langle T_{\Sigma}(X \cup\right.\right.\right.$ $\left.\left.\left.\left.Y_{k}\right)\right\rangle\right)^{Q \times Q^{k}}\right)^{T_{\Gamma}(Z) \times T_{\Gamma}(Z)^{k}}$ and with the final state vector $F$ in $\left(\left(A\left\langle T_{\Sigma}\left(X \cup Z_{Q}\right)\right\rangle\right)^{Q \times 1}\right)^{T_{\Gamma}(Z) \times 1}$.

Clearly, this means that all notions concerning tree automata (e. g., simple tree automata) are also notions for pushdown tree automata.

Observe that we have adapted the definition of a pushdown tree automaton as given in Kuich [43] to fit into our general definition of a polynomial tree automaton.

Consider now the polynomial system constructed from $\mathfrak{A}$ as in the proof of Theorem 3.3 and transfer it isomorphically to a system that "belongs" to $\mathfrak{P}$, i. e.,

$$
y=M(y, \ldots, y)+F .
$$

Here $y \in\left(\left\{\left(y_{t}\right)_{q} \mid t \in T_{\Gamma}(Z), q \in Q\right\}^{Q \times 1}\right)^{T_{\Gamma}(Z) \times 1}$ is a vector of variables $\left(y_{t}\right)_{q}$, $t \in T_{\Gamma}(Z), q \in Q$, such that $\left(y_{t}\right)_{q}$ is the $t$ - $q$-entry of $y$. 
The equations of the linear system $(*)$ are, in block notation, for $t \in T_{\Gamma}(Z)$,

$$
y_{t}=\sum_{t_{1}, \ldots, t_{k} \in T_{\Gamma}(Z)} M_{t,\left(t_{1}, \ldots, t_{k}\right)}\left(y_{t_{1}}, \ldots, y_{t_{k}}\right)+F_{t}
$$

where $y_{t}$ is a $Q \times 1$-vector, whose $q$-entry is the variable $\left(y_{t}\right)_{q}, q \in Q$; and for $t \in T_{\Gamma}(Z), q \in Q$,

$$
\begin{aligned}
&\left(y_{t}\right)_{q}=\sum_{t_{1}, \ldots, t_{k} \in T_{\Gamma}(Z)} \sum_{q_{1}, \ldots, q_{k} \in Q} \\
&\left(M_{t,\left(t_{1}, \ldots, t_{k}\right)}\right)_{q,\left(q_{1}, \ldots, q_{k}\right)}\left(\left(y_{t_{1}}\right)_{q_{1}}, \ldots,\left(y_{t_{k}}\right)_{q_{k}}\right)+\left(F_{t}\right)_{q} .
\end{aligned}
$$

Hence, for all $g \in \Gamma_{m}, 0 \leq m \leq \bar{m}$, and all $u_{1}, \ldots, u_{m} \in T_{\Gamma}\left(Z_{m}\right)$, the equations in matrix notation are

$$
\begin{aligned}
y_{g\left(u_{1}, \ldots, u_{m}\right)=} & \sum_{v_{1}, \ldots, v_{k} \in T_{\Gamma}\left(Z_{m}\right)} \\
& M_{g\left(z_{1}, \ldots, z_{m}\right),\left(v_{1}, \ldots, v_{k}\right)}\left(y_{v_{1}\left(u_{1}, \ldots, u_{m}\right)}, \ldots, y_{v_{k}\left(u_{1}, \ldots, u_{m}\right)}\right) \\
& +P_{g\left(z_{1}, \ldots, z_{m}\right)} .
\end{aligned}
$$

and, for $z_{i} \in Z$,

$$
y_{z_{i}}=F_{z_{i}} .
$$

Here $v_{i}\left(u_{1}, \ldots, u_{m}\right), 1 \leq i \leq k$, denotes $v_{i}\left[u_{j} / z_{j}, 1 \leq j \leq m\right]$. The least solution of this polynomial system is the least upper bound of the approximation sequence associated with $\mathfrak{P}$.

An example will illustrate the notions connected with pushdown tree automata. This example is already given in Kuich [43].

Example 5.1. (Guessarian [32], Example 3). The pushdown tree automaton $M$ of Example 3 of Guessarian [32] is specified by our concepts as follows: The input alphabet is $F=\left\{b, c_{1}, c_{2}\right\}, \operatorname{rank}(b)=2, \operatorname{rank}\left(c_{i}\right)=0, i=1,2 ; X$ is the empty set. $\mathfrak{P}=\left(Q, \Pi,\{z\},\left\{y_{1}, y_{2}\right\}, M, S, Z_{0}, P\right)$, where $Q=\left\{q_{0}, q_{1}, q_{2}\right\}$, $\Pi=\left\{G, C, Z_{0}\right\}, \operatorname{rank}(G)=1, \operatorname{rank}(C)=\operatorname{rank}\left(Z_{0}\right)=0, P=\left(P_{C}, P_{Z_{0}}, P_{G(z)}\right)$, and $M$ is defined by

(0) $\left(M_{Z_{0}, G(C)}\right)_{q_{0}, q_{0}}=y_{1}$,

(1) $\left(M_{G(z), G(G(z))}\right)_{q_{0}, q_{0}}=y_{1}$,

(2) $\left(M_{G(z),(z, z)}\right)_{q_{0},\left(q_{1}, q_{2}\right)}=b\left(y_{1}, y_{2}\right)$,

(3) $\left(M_{G(z),(z, z)}\right)_{q_{i},\left(q_{i}, q_{i}\right)}=b\left(y_{1}, y_{2}\right), i=1,2$,

(4) $\left(P_{C}\right)_{q_{i}}=c_{i}, i=1,2$.

All other entries of the $Z_{0}, C$ and $G(z)$ block row of $M_{1}$ and $M_{2}$ are zero; moreover, $\left(P_{C}\right)_{q_{0}}=0$ and $P_{Z_{0}}=0, P_{G(z)}=0$; furthermore, $S_{q_{0}}=y_{1}, S_{q_{1}}=$ $S_{q_{2}}=0$. 
The important entries of the vectors of the approximation sequence associated with $\mathfrak{P}$ are defined as follows for all $u \in T_{\Pi}(\{z\})$ and $j \geq 0$ :

$$
\begin{aligned}
& \left(\tau_{Z_{0}}^{j+1}\right)_{q_{0}}=\left(\tau_{G(C)}^{j}\right)_{q_{0}}, \quad\left(\tau_{Z_{0}}^{j+1}\right)_{q_{i}}=0, i=1,2 ; \\
& \left(\tau_{C}^{j+1}\right)_{q_{0}}=0,\left(\tau_{C}^{j+1}\right)_{q_{i}}=c_{i}, i=1,2 ;\left(\tau_{z}^{j+1}\right)_{q_{i}}=z_{q_{i}}, i=0,1,2 ; \\
& \left(\tau_{G(u)}^{j+1}\right)_{q_{0}}=\left(\tau_{G(G(u))}^{j}\right)_{q_{0}}+b\left(\left(\tau_{u}^{j}\right)_{q_{1}},\left(\tau_{u}^{j}\right)_{q_{2}}\right), \\
& \left(\tau_{G(u)}^{j+1}\right)_{q_{i}}=b\left(\left(\tau_{u}^{j}\right)_{q_{i}},\left(\tau_{u}^{j}\right)_{q_{i}}\right), i=1,2 .
\end{aligned}
$$

Let $G^{k}(C) \in T_{\Pi}(\emptyset)$ be defined by $G^{0}(C)=C, G^{k+1}(C)=G\left(G^{k}(C)\right), k \geq 0$, and consider the equations for $G^{k}(C), k \geq 0, j \geq 0, i=1,2$ :

$$
\begin{aligned}
& \left(\tau_{G^{0}(C)}^{j+1}\right)_{q_{0}}=0,\left(\tau_{G^{0}(C)}^{j+1}\right)_{q_{i}}=c_{i} \\
& \left(\tau_{G^{k}(C)}^{j+1}\right)_{q_{0}}=\left(\tau_{G^{k+1}(C)}^{j}\right)_{q_{0}}+b\left(\left(\tau_{G^{k-1}(C)}^{j}\right)_{q_{1}},\left(\tau_{G^{k-1}(C)}\right)_{q_{2}}\right), \\
& \left(\tau_{G^{k}(C)}\right)_{q_{i}}=b\left(\left(\tau_{G^{k-1}(C)}^{j}\right)_{q_{i}},\left(\tau_{G^{k-1}(C)}\right)_{q_{i}}\right) .
\end{aligned}
$$

Let $\tau=\sup \left(\tau^{j} \mid j \in \mathbb{N}\right)$. Then, for $k \geq 1, i=1,2$,

$$
\begin{aligned}
& \left(\tau_{G^{0}(C)}\right)_{q_{0}}=0,\left(\tau_{G^{0}(C)}\right)_{q_{i}}=c_{i} \\
& \left(\tau_{G^{k}(C)}\right)_{q_{0}}=\left(\tau_{G^{k+1}(C)}\right)_{q_{0}}+b\left(\left(\tau_{G^{k-1}(C)}\right)_{q_{1}},\left(\tau_{G^{k-1}(C)}\right)_{q_{2}}\right) \\
& \left(\tau_{G^{k}(C)}\right)_{q_{i}}=b\left(\left(\tau_{G^{k-1}(C)}\right)_{q_{i}},\left(\tau_{G^{k-1}(C)}\right)_{q_{i}}\right) .
\end{aligned}
$$

Hence $\left(\tau_{G^{k}(C)} \mid k \geq 0\right)$ is the least solution of the polynomial system

$$
\begin{aligned}
& \left(z_{0}\right)_{q_{0}}=0, \quad\left(z_{0}\right)_{q_{i}}=c_{i}, i=1,2 ; \\
& \left(z_{k}\right)_{q_{0}}=\left(z_{k+1}\right)_{q_{0}}+b\left(\left(z_{k-1}\right)_{q_{1}},\left(z_{k-1}\right)_{q_{2}}\right), k \geq 1, \\
& \left(z_{k}\right)_{q_{i}}=b\left(\left(z_{k-1}\right)_{q_{i}},\left(z_{k-1}\right)_{q_{i}}\right), \quad i=1,2, k \geq 1 .
\end{aligned}
$$

By Theorem 3.2, $\left(\tau_{G^{k}(C)} \mid k \geq 0\right)$ is also the least solution of the system

$$
\begin{aligned}
& \left(z_{0}\right)_{q_{0}}=0, \quad\left(z_{0}\right)_{q_{i}}=c_{i}, i=1,2 ; \\
& \left(z_{k}\right)_{q_{0}}=\sum_{j \geq k-1} b\left(\left(z_{j}\right)_{q_{1}},\left(z_{j}\right)_{q_{2}}\right), k \geq 1, \\
& \left(z_{k}\right)_{q_{i}}=b\left(\left(z_{k-1}\right)_{q_{i}},\left(z_{k-1}\right)_{q_{i}}\right), i=1,2, k \geq 1 .
\end{aligned}
$$

This system is proper and has the unique solution $\left(\tau_{G^{k}(C)} \mid k \geq 0\right)$. Observe that this system is not polynomial.

Define now the trees $t_{i}^{j} \in T_{F}(\emptyset), i=1,2, j \geq 0$, by

$$
t_{i}^{0}=c_{i}, \quad t_{i}^{j+1}=b\left(t_{i}^{j}, t_{i}^{j}\right), i=1,2, j \geq 0 .
$$

Let

$$
\left(s_{k}\right)_{q_{0}}=\sum_{j \geq k-1} b\left(t_{1}^{j}, t_{2}^{j}\right),\left(s_{k}\right)_{q_{i}}=t_{i}^{k}, k \geq 1,\left(s_{0}\right)_{q_{0}}=0,\left(s_{0}\right)_{q_{i}}=c_{i}, i=1,2 .
$$


Then $\left(\left(s_{k}\right)_{q_{i}} \mid k \geq 0, i=0,1,2\right)$ is a solution of this proper system and, hence, $\left(s_{k}\right)_{q_{i}}=\left(\tau_{G^{k}(C)}\right)_{q_{i}}, k \geq 0, i=0,1,2$. Since $\|\mathfrak{P}\|=\left(\tau_{Z_{0}}\right)_{q_{0}}=\left(\tau_{G(C)}\right)_{q_{0}}$, we infer that $\|\mathfrak{P}\|=\left(s_{1}\right)_{q_{0}}=\sum_{j>0} b\left(t_{1}^{j}, t_{2}^{j}\right)$.

This example indicates also a method to prove in a mathematically rigorous manner that the behavior of a pushdown tree automaton equals a certain formal tree series.

We now will refer to a result for pushdown tree automata that is analogous to Theorem 6.2 of Kuich [36] for pushdown automata. Intuitively, it states that the computations of the pushdown tree automaton governed by a pushdown tape with contents $t\left(u_{1}, \ldots, u_{m}\right)$ (i. e., $\left.\tau_{t\left(u_{1}, \ldots, u_{m}\right)}\right)$, where $t\left(z_{1}, \ldots, z_{m}\right) \in T_{\Gamma}\left(Z_{m}\right)$ and $u_{i} \in T_{\Gamma}\left(Z_{m}\right), 1 \leq i \leq m$, are the same as the computations governed by a pushdown tape with contents $t\left(z_{1}, \ldots, z_{m}\right)$ (i. e., $\left.\tau_{t\left(z_{1}, \ldots, z_{m}\right)}\right)$ applied to the computations governed by pushdown tapes with contents $u_{1}, \ldots, u_{m}$ (i. e., $\left.\tau_{t\left(z_{1}, \ldots, z_{m}\right)}\left[\tau_{u_{i}} / F_{z_{i}}, 1 \leq i \leq m\right]\right)$.

Theorem 5.1 Let $\tau$ be the least solution of the polynomial linear system (*). Then, for all $t\left(z_{1}, \ldots, z_{m}\right) \in T_{\Gamma}\left(Z_{m}\right), 1 \leq m \leq \bar{m}$, and $u_{i} \in T_{\Gamma}\left(Z_{m}\right), 1 \leq i \leq m$,

$$
\tau_{t\left(u_{1}, \ldots, u_{m}\right)}=\tau_{t\left(z_{1}, \ldots, z_{m}\right)}\left[\tau_{u_{i}} / F_{z_{i}}, 1 \leq i \leq m\right] .
$$

We now introduce algebraic tree systems. The definitions follow Kuich [43]. Let $\Phi=\left\{G_{1}, \ldots, G_{n}\right\}, \Phi \cap \Sigma=\emptyset$, be a finite ranked alphabet of function variables, where $G_{i}$ has rank $r_{i}, 1 \leq i \leq n$, and $\bar{m}=\max \left\{r_{i} \mid 1 \leq i \leq n\right\}$.

Let $D=A\left\langle\left\langle T_{\Sigma}\left(X \cup Z_{r_{1}}\right)\right\rangle\right\rangle \times \ldots \times A\left\langle\left\langle T_{\Sigma}\left(X \cup Z_{r_{n}}\right)\right\rangle\right\rangle$ and consider tree series $s_{i} \in A\left\langle\left\langle T_{\Sigma \cup \Phi}\left(X \cup Z_{r_{i}}\right)\right\rangle\right\rangle, 1 \leq i \leq n$. Then each $s_{i}$ induces a function $\bar{s}_{i}: D \rightarrow$ $A\left\langle\left\langle T_{\Sigma}\left(X \cup Z_{r_{i}}\right)\right\rangle\right\rangle$. For $\left(\tau_{1}, \ldots, \tau_{n}\right) \in D$, we define inductively $\bar{s}_{i}\left(\tau_{1}, \ldots, \tau_{n}\right)$ to be

(i) $z_{m}$ if $s_{i}=z_{m}, 1 \leq m \leq r_{i} ; x$ if $s_{i}=x, x \in X$;

(ii) $\bar{\omega}\left(\bar{t}_{1}\left(\tau_{1}, \ldots, \tau_{n}\right), \ldots, \bar{t}_{r}\left(\tau_{1}, \ldots, \tau_{n}\right)\right)$ if $s_{i}=\omega\left(t_{1}, \ldots, t_{r}\right), \omega \in \Sigma_{r}$, $t_{1}, \ldots, t_{r} \in T_{\Sigma \cup \Phi}\left(X \cup Z_{r_{i}}\right) ;$

(iii) $\tau_{j}\left(\bar{t}_{1}\left(\tau_{1}, \ldots, \tau_{n}\right), \ldots, \bar{t}_{r_{j}}\left(\tau_{1}, \ldots, \tau_{n}\right)\right)$ if $s_{i}=G_{j}\left(t_{1}, \ldots, t_{r_{j}}\right), G_{j} \in \Phi$, $t_{1}, \ldots, t_{r_{j}} \in T_{\Sigma \cup \Phi}\left(X \cup Z_{r_{i}}\right)$;

(iv) $a \bar{t}\left(\tau_{1}, \ldots, \tau_{n}\right)$ if $s_{i}=a t, a \in A, t \in T_{\Sigma \cup \Phi}\left(X \cup Z_{r_{i}}\right)$;

(v) $\sum_{j \in J} \bar{r}_{j}\left(\tau_{1}, \ldots, \tau_{n}\right)$ if $s_{i}=\sum_{j \in J} r_{j}, r_{j} \in A\left\langle\left\langle T_{\Sigma \cup \Phi}\left(X \cup Z_{r_{i}}\right)\right\rangle\right\rangle, j \in J$, for an arbitrary index set $J$.

The mappings $\bar{s}_{i}, 1 \leq i \leq n$, and the mapping $\bar{s}: D \rightarrow D$, where $\bar{s}=$ $\left\langle\bar{s}_{1}, \ldots, \bar{s}_{k}\right\rangle$ are continuous. This can be shown for $s_{i} \in T_{\Sigma \cup \Phi}\left(X \cup Z_{r}\right)$ by induction on the structure of $s_{i}$. The case that $s_{i}$ is of the form $G_{j}\left(t_{1}, \ldots, t_{r_{j}}\right)$ follows from the continuity of substitution as shown by Proposition 2.6. Since scalar multiplication is continuous, it follows now that each $s_{i}=a t$, where 
$a \in A$ and $t \in T_{\Sigma \cup \Phi}\left(X \cup Z_{r}\right)$ also induces a continuous function. The general case $s_{i} \in A\left\langle\left\langle T_{\Sigma \cup \Phi}\left(X \cup Z_{r}\right)\right\rangle\right\rangle$ can now be handled using the fact that summations preserve least upper bounds of directed sets. Hence, $\bar{s}$ has a least fixed point in D. (See also Lemmas 4.24 and 4.3 of Guessarian [31]; Lemmas 3.1 and 3.2 of Engelfriet, Schmidt [20]; Bloom, Ésik [6]; Ésik [21].) In certain situations, formulae are easier to read if we use the notation $s_{i}\left[\tau_{1} / G_{1}, \ldots, \tau_{n} / G_{n}\right]$ instead of the notation $\bar{s}_{i}\left(\tau_{1}, \ldots, \tau_{n}\right)$.

An algebraic tree system $\mathfrak{S}=(\Phi, Z, \Sigma, E)$ (with function variables in $\Phi$, variables in $Z$ and terminal symbols in $\Sigma$ ) has a set $E$ of formal equations

$$
G_{i}\left(z_{1}, \ldots, z_{r_{i}}\right)=s_{i}\left(z_{1}, \ldots, z_{r_{i}}\right), \quad 1 \leq i \leq n
$$

where each $s_{i}$ is in $A\left\langle T_{\Sigma \cup \Phi}\left(X \cup Z_{r_{i}}\right)\right\rangle$. A solution to the algebraic tree system $\mathfrak{S}$ is given by $\left(\tau_{1}, \ldots, \tau_{n}\right) \in D$ such that $\tau_{i}=\bar{s}_{i}\left(\tau_{1}, \ldots, \tau_{n}\right), 1 \leq i \leq n$, i. e., by any fixed point $\left(\tau_{1}, \ldots, \tau_{n}\right)$ of $\bar{s}=\left\langle\bar{s}_{1}, \ldots, \bar{s}_{n}\right\rangle$. A solution $\left(\sigma_{1}, \ldots, \sigma_{n}\right)$ of the algebraic tree system $\mathfrak{S}$ is called least solution iff $\sigma_{i} \sqsubseteq \tau_{i}, 1 \leq i \leq n$, for all solutions $\left(\tau_{1}, \ldots, \tau_{n}\right)$ of $\mathfrak{S}$. Since the least solution of $\mathfrak{S}$ is nothing else than the least fixed point of $\bar{s}=\left\langle\bar{s}_{1}, \ldots, \bar{s}_{n}\right\rangle$, the least solution of the algebraic system $\mathfrak{S}$ exists in $D$. (See Wechler [59], Section 1.5.)

Theorem 5.2 Let $\mathfrak{S}=\left(\Phi, Z, \Sigma,\left\{G_{i}=s_{i} \mid 1 \leq i \leq n\right\}\right)$ be an algebraic tree system, where $s_{i} \in A\left\langle T_{\Sigma \cup \Phi}\left(X \cup Z_{r_{i}}\right)\right\rangle$. Then the least solution of this algebraic tree system $\mathfrak{S}$ exists in $D$ and equals

$$
\operatorname{fix}(\bar{s})=\sup \left(\bar{s}^{j}(0) \mid j \in \mathbb{N}\right),
$$

where $\bar{s}^{j}$ is the $j$-th iterate of the mapping $\bar{s}=\left\langle\bar{s}_{1}, \ldots, \bar{s}_{n}\right\rangle: D \rightarrow D$.

Theorem 5.2 indicates how we can compute an approximation to the least solution of an algebraic tree system. The approximation sequence $\left(\tau^{j} \mid j \in \mathbb{N}\right)$, where each $\tau^{j} \in D$, associated with the algebraic tree system $\mathfrak{S}=\left(\Phi, Z, \Sigma,\left\{G_{i}=s_{i} \mid\right.\right.$ $1 \leq i \leq n\})$ is defined as follows:

$$
\tau^{0}=0, \quad \tau^{j+1}=\bar{s}\left(\tau^{j}\right), j \in \mathbb{N} .
$$

Clearly, the least solution $\operatorname{fix}(\bar{s})$ of $\mathfrak{S}$ is equal to $\sup \left(\tau^{j} \mid j \in \mathbb{N}\right)$. An algebraic tree system $\mathfrak{S}=\left(\Phi, Z, \Sigma,\left\{G_{i}=s_{i} \mid 0 \leq i \leq n\right\}, G_{0}\right)$ (with function variables in $\Phi=\left\{G_{0}, G_{1}, \ldots, G_{n}\right\}$, variables in $Z$, terminal symbols in $\Sigma$ ) with initial function variable $G_{0}$ is an algebraic tree system $\left(\Phi, Z, \Sigma,\left\{G_{i}=s_{i} \mid 0 \leq i \leq n\right\}\right)$ such that $G_{0}$ has rank 0 . Let $\left(\tau_{0}, \tau_{1}, \ldots, \tau_{n}\right)$ be the least solution of $\left(\Phi, Z, \Sigma,\left\{G_{i}=\right.\right.$ $\left.\left.s_{i} \mid 0 \leq i \leq n\right\}\right)$. Then $\tau_{0}$ is called the initial component of the least solution. Observe that $\tau_{0} \in A\left\langle\left\langle T_{\Sigma}(X)\right\rangle\right\rangle$ contains no variables of $Z$.

Our algebraic tree systems are second-order systems in the sense of Bozapalidis [11] and are a generalization of the context-free tree grammars. (See Rounds [51], and Engelfriet, Schmidt [20], especially Theorem 3.4.) 
A tree series in $A\left\langle\left\langle T_{\Sigma}(X)\right\rangle\right\rangle$ is called algebraic iff it is the initial component of the least solution of an algebraic tree system with initial function variable. The collection of all these initial components is denoted by $A^{\text {alg }}\left\langle\left\langle T_{\Sigma}(X)\right\rangle\right\rangle$. There is no restriction of the alphabets $\Sigma$ and $X$ in the definiton of an algebraic tree series, i. e., they may be infinite. This is due to the fact that, for $s \in A^{\text {alg }}\left\langle\left\langle T_{\Sigma}(X)\right\rangle\right\rangle$ there exist finite alphabets $\Sigma^{\prime}$ and $X^{\prime}, \Sigma^{\prime} \subseteq \Sigma, X^{\prime} \subseteq X$, such that $\operatorname{supp}(s) \subseteq T_{\Sigma^{\prime}}\left(X^{\prime}\right)$. Moreover,

$$
A^{\text {alg }}\left\langle\left\langle T_{\Sigma}(X)\right\rangle\right\rangle=\bigcup_{\Sigma^{\prime} \subseteq \Sigma \text { finite, } X^{\prime} \subseteq X \text { finite }} A^{\text {alg }}\left\langle\left\langle T_{\Sigma^{\prime}}\left(X^{\prime}\right)\right\rangle\right\rangle .
$$

Given a pushdown tree automaton $\mathfrak{P}=\left(Q, \Gamma, Z, Y, M, S, p_{0}, P\right)$, we now construct an equivalent algebraic tree system $\mathfrak{S}=\left(\Phi, Z_{Q}, \Sigma, E, y_{0}\right)$ with initial function variable $y_{0}$. (The construction follows Kuich [43].) Here $\Phi=$ $\left\{y_{0}\right\} \cup\left\{\left(y_{g\left(z_{1}, \ldots, z_{m}\right)}\right)_{q} \mid g \in \Gamma_{m}, 0 \leq m \leq \bar{m}, q \in Q\right\}$. The function variable $\left(y_{g\left(z_{1}, \ldots, z_{m}\right)}\right)_{q}, g \in \Gamma_{m}, 0 \leq m \leq \bar{m}, q \in Q$, has the rank $m|Q|$. By definition, the $Q \times 1$-vector $y_{g\left(z_{1}, \ldots, z_{m}\right)}, g \in \Gamma_{m}, 0 \leq m \leq \bar{m}$, is the column vector with $q$-component $\left(y_{g\left(z_{1}, \ldots, z_{m}\right)}\right)_{q}, q \in Q$.

For the specification of the formal equations in $E$ we have to introduce, for $t \in T_{\Gamma}\left(Z_{m}\right), 1 \leq m \leq \bar{m}$, vectors $\hat{y}_{t}$ in $\left(T_{\Phi}\left(Z_{Q}^{m}\right)\right)^{Q \times 1}$ as follows:

$$
\begin{aligned}
& \hat{y}_{g\left(u_{1}, \ldots, u_{m}\right)}=y_{g\left(z_{1}, \ldots, z_{m}\right)}\left(\hat{y}_{u_{1}}, \ldots, \hat{y}_{u_{m}}\right), \\
& \quad g \in \Gamma_{m}, u_{1}, \ldots, u_{m} \in T_{\Gamma}\left(Z_{m}\right), 1 \leq m \leq \bar{m} ; \\
& \hat{y}_{g}=y_{g}, g \in \Gamma_{0} ; \quad\left(\hat{y}_{z_{i}}\right)_{q}=\left(z_{i}\right)_{q}, 1 \leq i \leq \bar{m}, q \in Q .
\end{aligned}
$$

Written componentwise, the first equation reads

$$
\left(\hat{y}_{g\left(u_{1}, \ldots, u_{m}\right)}\right)_{q}=\left(y_{g\left(z_{1}, \ldots, z_{m}\right)}\right)_{q}\left(\left(\hat{y}_{u_{i}}\right)_{q^{\prime}}, 1 \leq i \leq m, q^{\prime} \in Q\right)
$$

for $g \in \Gamma_{m}, u_{1}, \ldots, u_{m} \in T_{\Gamma}\left(Z_{m}\right), 1 \leq m \leq \bar{m}, q \in Q$. Observe that

$$
\left(\hat{y}_{g\left(z_{1}, \ldots, z_{m}\right)}\right)_{q}=\left(y_{g\left(z_{1}, \ldots, z_{m}\right)}\right)_{q}\left(\left(z_{i}\right)_{q^{\prime}}, 1 \leq i \leq m, q^{\prime} \in Q\right)
$$

for $g \in \Gamma_{m}, 1 \leq m \leq \bar{m}, q \in Q$. Observe further that $y_{g\left(z_{1}, \ldots, z_{m}\right)}\left(\hat{y}_{u_{1}}, \ldots, \hat{y}_{u_{m}}\right)$ means $y_{g\left(z_{1}, \ldots, z_{m}\right)}\left[\hat{y}_{u_{i}} / F_{z_{i}}, 1 \leq i \leq m\right]$ and $\left(y_{g\left(z_{1}, \ldots, z_{m}\right)}\right)_{q}\left(\left(\hat{y}_{i}\right)_{q^{\prime}}, 1 \leq i \leq m, q^{\prime} \in\right.$ Q) means $\left(y_{g\left(z_{1}, \ldots, z_{m}\right)}\right)_{q}\left[\left(\hat{y}_{i}\right)_{q^{\prime}} /\left(z_{i}\right)_{q^{\prime}}, 1 \leq i \leq m, q^{\prime} \in Q\right]$. The formal equations in $E$ are now given in matrix notation:

$$
\begin{aligned}
& y_{0}=S\left(y_{p_{0}}\right), \\
& y_{g\left(z_{1}, \ldots, z_{m}\right)}\left(\left(z_{i}\right)_{q^{\prime}}, 1 \leq i \leq m, q^{\prime} \in Q\right)= \\
& \quad\left(\sum_{1 \leq k \leq \bar{k}} M_{k}(\hat{y}, \ldots, \hat{y})+F\right)_{g\left(z_{1}, \ldots, z_{m}\right)}= \\
& \quad \sum_{1 \leq k \leq \bar{k}} \sum_{t_{1}, \ldots, t_{k} \in T_{\Gamma}\left(Z_{m}\right)}\left(M_{k}\right)_{g\left(z_{1}, \ldots, z_{m}\right),\left(t_{1}, \ldots, t_{k}\right)}\left(\hat{y}_{t_{1}}, \ldots, \hat{y}_{t_{k}}\right)+P_{g\left(z_{1}, \ldots, z_{m}\right)}, \\
& g \in \Gamma_{m}, 0 \leq m \leq \bar{m} .
\end{aligned}
$$


We now give explicitely the formal equations, except the first one, with index $q \in$ $Q$. Observe that indexing by $q \in Q$ is needed only in examples. In theoretical considerations, we save the indexing by states $q, q_{1}, \ldots, q_{n}$, i. e., we use the form given above.

$$
\begin{aligned}
& \left(y_{g\left(z_{1}, \ldots, z_{m}\right)}\right)_{q}\left(\left(z_{i}\right)_{q^{\prime}}, 1 \leq i \leq m, q^{\prime} \in Q\right)= \\
& \begin{array}{l}
\sum_{1 \leq k \leq \bar{k}} \sum_{t_{1}, \ldots, t_{k} \in T_{\Gamma}\left(Z_{m}\right)} \sum_{q_{1}, \ldots, q_{k} \in Q} \\
\quad\left(\left(M_{k}\right)_{g\left(z_{1}, \ldots, z_{m}\right),\left(t_{1}, \ldots, t_{k}\right)}\right)_{q,\left(q_{1}, \ldots, q_{k}\right)}\left(\left(\hat{y}_{t_{1}}\right)_{q_{1}}, \ldots,\left(\hat{y}_{t_{k}}\right)_{q_{k}}\right)+\left(P_{g\left(z_{1}, \ldots, z_{m}\right)}\right)_{q}, \\
g \in \Gamma_{m}, 0 \leq m \leq \bar{m}, q \in Q .
\end{array}
\end{aligned}
$$

We denote this system of formal equations by $(* *)$.

The next theorem is a key result for proving the equivalence of pushdown tree automata and algebraic tree systems with initial function variable.

Theorem 5.3 If $\tau$ is the least solution of the polynomial linear system $(*)$ then $\left(\tau_{g\left(z_{1}, \ldots, z_{m}\right)} \mid g \in \Gamma_{m}, 0 \leq m \leq \bar{m}\right)$ is the least solution of the algebraic tree system $(* *)$.

Corollary 5.4 The initial component of the least solution of the algebraic tree system $\mathfrak{S}$ coincides with $\|\mathfrak{P}\|$.

Corollary 5.5 The behavior of a pushdown tree automaton is an algebraic tree series.

Example 5.1 (continued). We now construct step-by-step for the pushdown tree automaton $\mathfrak{P}$ the algebraic tree system $\mathfrak{S}$ with initial function variable such that $\|\mathfrak{P}\|$ is the initial component of its least solution. We first consider the linear system $(*)$ written in the form

$$
\hat{y}=M(\hat{y}, \hat{y})+F
$$

and write down explicitely the equations for $\hat{y}_{G(z)}, \hat{y}_{Z_{0}}$ and $\hat{y}_{C}$ :

$$
\begin{aligned}
& \left(\hat{y}_{G(z)}\right)_{q_{0}}=\left(\hat{y}_{G(G(z))}\right)_{q_{0}}+b\left(\left(\hat{y}_{z}\right)_{q_{1}},\left(\hat{y}_{z}\right)_{q_{2}}\right), \\
& \left(\hat{y}_{G(z)}\right)_{q_{i}}=b\left(\left(\hat{y}_{z}\right)_{q_{i}},\left(\hat{y}_{z}\right)_{q_{i}}\right), i=1,2, \\
& \left(\hat{y}_{Z_{0}}\right)_{q_{0}}=\left(\hat{y}_{G(C)}\right)_{q_{0}}, \quad\left(\hat{y}_{Z_{0}}\right)_{q_{i}}=0, i=1,2, \\
& \left(\hat{y}_{C}\right)_{q_{0}}=0, \quad\left(\hat{y}_{C}\right)_{q_{i}}=c_{i}, i=1,2 .
\end{aligned}
$$

Now we express the components of $\hat{y}$ by $y_{G(z)}, y_{Z_{0}}$ and $y_{C}$; and obtain the algebraic system $(* *)$ :

$$
\begin{aligned}
& \left(y_{G(z)}\right)_{q_{0}}\left(z_{q_{0}}, z_{q_{1}}, z_{q_{2}}\right)=\left(y_{G(z)}\right)_{q_{0}}\left(\left(y_{G(z)}\right)_{q_{0}}\left(z_{q_{0}}, z_{q_{1}}, z_{q_{2}}\right),\right. \\
& \left.\left(y_{G(z)}\right)_{q_{1}}\left(z_{q_{0}}, z_{q_{1}}, z_{q_{2}}\right),\left(y_{G(z)}\right)_{q_{2}}\left(z_{q_{0}}, z_{q_{1}}, z_{q_{2}}\right)\right)+b\left(z_{q_{1}}, z_{q_{2}}\right), \\
& \left(y_{G(z)}\right)_{q_{i}}\left(z_{q_{0}}, z_{q_{1}}, z_{q_{2}}\right)=b\left(z_{q_{i}}, z_{q_{i}}\right), i=1,2, \\
& \left(y_{Z_{0}}\right)_{q_{0}}=\left(y_{G(z)}\right)_{q_{0}}\left(\left(y_{C}\right)_{q_{0}},\left(y_{C}\right)_{q_{1}},\left(y_{C}\right)_{q_{2}}\right), \\
& \left(y_{Z_{0}}\right)_{q_{i}}=0, i=1,2, \\
& \left(y_{C}\right)_{q_{0}}=0, \\
& \left(y_{C}\right)_{q_{i}}=c_{i}, i=1,2 .
\end{aligned}
$$


The algebraic tree system $\mathfrak{S}=\left(\Phi, Z, F, E, y_{0}\right)$ is now specified by $\Phi=\left\{\left(y_{G(z)}\right)_{q_{i}},\left(y_{Z_{0}}\right)_{q_{i}},\left(y_{C}\right)_{q_{i}} \mid i=0,1,2\right\} \cup\left\{y_{0}\right\}$, where the ranks of $\left(y_{G(z)}\right) q_{i}$, $\left(y_{Z_{0}}\right)_{q_{i}},\left(y_{C}\right)_{q_{i}}$ are $3,0,0$, respectively, for $i=0,1,2$;

$Z=\left\{z_{q_{0}}, z_{q_{1}}, z_{q_{2}}\right\}$;

$E$ is the set of equations specified above augmented by the additional equation $y_{0}=\left(y_{Z_{0}}\right)_{q_{0}}$.

Observe that the construction of $\mathfrak{P}$ from $\mathfrak{S}$ is essentially the same construction as given by Guessarian [32] in her proof of Theorem 1.

The converse of Corollary 5.5 can be proved and yields the main result of Kuich [43]. It is also the main result of this section.

Corollary 5.6 The following statements on a formal tree series $s$ in $A\left\langle\left\langle T_{\Sigma}(X)\right\rangle\right\rangle$ are equivalent:

(i) $s$ is an algebraic tree series;

(ii) $s$ is the behavior of a pushdown tree automaton;

(iii) $s$ is the behavior of a simple pushdown tree automaton with one initial state of weight 1 .

If our basic semiring is $\mathbb{N}^{\infty}$, i. e., if we consider tree series in $\mathbb{N}^{\infty}\left\langle\left\langle T_{\Sigma}(X)\right\rangle\right\rangle$, we can draw some stronger conclusions.

Let $G=(\Phi, Z, \Sigma, R)$ be a context-free tree grammar, where $\Phi=\left\{G_{1}, \ldots, G_{n}\right\}$ and $R$ is the set of rules

$$
G_{i}\left(z_{1}, \ldots, z_{r_{i}}\right) \rightarrow t_{i}^{j}, \quad 1 \leq j \leq n_{i}, 1 \leq i \leq n .
$$

Denote by $d_{i}(t), 1 \leq i \leq n$, the number (possibly $\infty$ ) of distinct leftmost derivations of $t \in T_{\Sigma}\left(X \cup Z_{r_{i}}\right)$ with respect to $G$ and starting from $G_{i}$. Let $\mathfrak{S}=(\Phi, Z, \Sigma, E)$ be an algebraic tree system, where $E$ is the set of formal equations

$$
G_{i}\left(z_{1}, \ldots, z_{r_{i}}\right)=\sum_{1 \leq j \leq n_{i}} t_{i}^{j}, \quad 1 \leq i \leq n .
$$

Then there exists the following theorem.

Theorem 5.7 (Bozapalidis [11], Theorem 11ii)) Let $G=(\Phi, Z, \Sigma, R)$ and $\mathfrak{S}=(\Phi, Z, \Sigma, E)$ be the context-free tree grammar and the algebraic tree system, respectively, considered above. Let $d_{i}(t), 1 \leq i \leq n$, be the number (possibly $\infty$ ) of distinct leftmost derivations of $t \in T_{\Sigma}\left(X \cup Z_{r_{i}}\right)$ with respect to $G$ and starting from $G_{i}$. Then the least solution of $\mathfrak{S}$ is given by

$$
\left(\sum_{t \in T_{\Sigma}\left(X \cup Z_{r_{i}}\right)} d_{i}(t) t \mid 1 \leq i \leq n\right) .
$$

Theorems 5.7, 3.1 and Corollary 5.6 yield the following theorem. 
Theorem 5.8 Let $d: T_{\Sigma}(X) \rightarrow \mathbb{N}^{\infty}$. Then the following statements are equivalent:

(i) There exists a context-free tree grammar with initial function variable, and with terminal alphabet $\Sigma$ and leaf alphabet $X$ such that the number $($ possibly $\infty)$ of distinct leftmost derivations of $t \in T_{\Sigma}(X)$ from the initial function variable is given by $d(t)$.

(ii) There exists a 1-simple pushdown tree automaton with input alphabet $\Sigma$ and leaf alphabet $X$ such that the number (possibly $\infty$ ) of distinct computations for $t \in T_{\Sigma}(X)$ is given by $d(t)$.

A context-free tree grammar with initial function variable, and with terminal alphabet $\Sigma$ and leaf alphabet $X$ is called unambiguous iff, for all $t \in T_{\Sigma}(X)$, the number of distinct leftmost derivations of $t$ with respect to $G$ is either 1 or 0. A 1-simple pushdown tree automaton with terminal alphabet $\Sigma$ and leaf alphabet $X$ is called unambiguous iff, for all $t \in T_{\Sigma}(X)$, the number of distinct computations for $t$ is either 1 or 0 .

Corollary 5.9 Let $L \subseteq T_{\Sigma}(X)$ be a tree language. Then $L$ is generated by an unambiguous context-free tree grammar iff $\sum_{t \in L} t$ is the behavior of an unambiguous 1-simple pushdown tree automaton.

A pushdown tree automaton $\mathfrak{P}=\left(Q, \Gamma, Z, Y, M, S, p_{0}, P\right)$ is called restricted iff $\Gamma=\left\{p_{0}\right\} \cup \Gamma_{1}$, i. e., except for the initial pushdown symbol $p_{0}$ of rank 0 , all other pushdown symbols have rank 1 .

The next theorem augments the list of equivalent statements of Corollary 5.6.

Theorem 5.10 The following statements on a formal tree series $s$ in $A\left\langle\left\langle T_{\Sigma}(X)\right\rangle\right\rangle$ are equivalent

(i) $s$ is an algebraic tree series;

(ii) $s$ is the behavior of a restricted pushdown tree automaton;

(iii) $s$ is the behavior of a simple restricted pushdown tree automaton.

We now turn to formal tree series in $\mathbb{N}^{\infty}\left\langle\left\langle T_{\Sigma}(X)\right\rangle\right\rangle$.

Theorem 5.11 Let $d: T_{\Sigma}(X) \rightarrow \mathbb{N}^{\infty}$. Then the following statement is equivalent to the statements of Theorem 5.9:

(iii) There exists a 1-simple restricted pushdown tree automaton with input alphabet $\Sigma$ and leaf alphabet $X$ such that the number (possibly $\infty$ ) of distinct computations for $t \in T_{\Sigma}(X)$ is given by $d(t)$.

Corollary 5.12 Let $L \subseteq T_{\Sigma}(X)$ be a tree language. Then $L$ is generated by an unambiguous context-free tree grammar iff $\sum_{t \in L} t$ is the behavior of an unambiguous 1-simple restricted pushdown tree automaton. 
We now prove a Kleene Theorem due to Bozapalidis [11]. For the remainder of this paper, $\Phi_{\infty}=\left\{G_{i} \mid i \geq 0\right\}$ denotes an infinite ranked alphabet of function variables, where $G_{i}$ has $\operatorname{rank} r_{i}, i \geq 0$, and for each $r \geq 0$ there are infinitely many function variables with rank $r$. Let $\hat{\Sigma}=\Sigma \cup\left\{G_{k_{1}}, \ldots, G_{k_{m}}\right\}$ and $\hat{D}=A\left\langle\left\langle T_{\hat{\Sigma}}\left(X \cup Z_{r_{i_{1}}}\right)\right\rangle\right\rangle \times \cdots \times A\left\langle\left\langle T_{\hat{\Sigma}}\left(X \cup Z_{r_{i_{n}}}\right)\right\rangle\right\rangle$ for some mutually different $i_{1}, \ldots, i_{n}, k_{1}, \ldots, k_{m} \geq 0$. Consider a tree series $s \in A\left\langle\left\langle T_{\hat{\Sigma} \cup\left\{G_{i_{1}}, \ldots, G_{i_{n}}\right\}}(X \cup\right.\right.$ $\left.\left.\left.Z_{r}\right)\right\rangle\right\rangle$. (The function variables $G_{k_{1}}, \ldots, G_{k_{m}}$ are considered here to be ranked symbols like in $\Sigma$.) Then $s$ induces a function $\bar{s}: \hat{D} \rightarrow A\left\langle\left\langle T_{\hat{\Sigma}}\left(X \cup Z_{r}\right)\right\rangle\right\rangle$ as defined above.

We now consider an algebraic tree system $\mathfrak{S}=\left(\left\{G_{i_{1}}, \ldots, G_{i_{n}}\right\}, Z, \hat{\Sigma}, E\right)$, where $E$ is $G_{i_{j}}\left(z_{1}, \ldots, z_{r_{i_{j}}}\right)=s_{j}\left(z_{1}, \ldots, z_{r_{i_{j}}}, G_{i_{1}}, \ldots, G_{i_{n}}\right), 1 \leq j \leq n$, and $s_{j} \in A\left\langle T_{\hat{\Sigma} \cup\left\{G_{i_{1}}, \ldots, G_{i_{n}}\right\}}\left(X \cup Z_{r_{i_{j}}}\right)\right\rangle$.

The least solution of $\mathfrak{S}$ is in $\hat{D}$. The collection of components of least solutions of all such algebraic systems (with free choice of mutually different $\left.i_{1}, \ldots, i_{n}, k_{1}, \ldots, k_{m} \geq 0\right)$ is denoted by $A^{\text {alg }}\left\langle\left\langle T_{\Sigma \cup \Phi_{\infty}}(X \cup Z)\right\rangle\right\rangle$. Observe that each power series in $A^{\text {alg }}\left\langle\left\langle T_{\Sigma \cup \Phi_{\infty}}(X \cup Z)\right\rangle\right\rangle$ is in fact a power series in $A^{\text {alg }}\left\langle\left\langle T_{\Sigma \cup \Phi}\left(X \cup Z_{r}\right)\right\rangle\right\rangle$ for some finite $\Phi \subset \Phi_{\infty}$ and some $r \geq 0$.

Before proving our results we apply a few results of the fixed point theory of continuous functions to algebraic tree systems. An extended algebraic tree system $\mathfrak{S}=(\Phi, Z, \Sigma, E)$ and its least solution are defined as an algebraic tree system and its least solution with the exception that the right sides of the equations $G_{i}\left(z_{1}, \ldots, z_{r_{i}}\right)=s_{i}\left(z_{1}, \ldots, z_{r_{i}}\right), 1 \leq i \leq n$, are now in $A\left\langle\left\langle T_{\Sigma \cup \Phi}(X \cup\right.\right.$ $\left.\left.Z_{r_{i}}\right)\right\rangle$.

(1) The parameter identity. Let $r \in A\left\langle\left\langle T_{\Sigma \cup \Phi_{\infty}}(X \cup Z)\right\rangle\right.$, and denote $r^{\prime}=$ $\mu G . r, G \in \Phi_{\infty}$. Let $G_{i} \neq G, \tau_{i} \in A\left\langle\left\langle T_{\Sigma \cup\left(\Phi_{\infty}-\{G\}\right)}(X \cup Z)\right\rangle\right\rangle, 1 \leq i \leq n$, and $\sigma_{j} \in A\left\langle\left\langle T_{\Sigma}(X \cup Z)\right\rangle\right\rangle, 1 \leq j \leq k$. Then $r^{\prime}\left[\sigma_{1} / z_{1}, \ldots, \sigma_{k} / z_{k}, \tau_{1} / G_{1}, \ldots, \tau_{n} / G_{n}\right]=$ $\mu G .\left(r\left[\sigma_{1} / z_{1}, \ldots, \sigma_{k} / z_{k}, \tau_{1} / G_{1}, \ldots, \tau_{n} / G_{n}\right]\right)$.

(2) The Bekić-De Bakker-Scott rule. Consider the equations $G_{i}\left(z_{1}, \ldots, z_{r_{i}}\right)=$ $s_{i}\left(z_{1}, \ldots, z_{r_{i}}\right), 1 \leq i \leq n, s_{i} \in A\left\langle\left\langle T_{\Sigma \cup \Phi}\left(X \cup Z_{r_{i}}\right)\right\rangle\right\rangle$ of an extended algebraic tree system $\mathfrak{S}=(\Phi, Z, \Sigma, E)$, where $\Phi=\left\{G_{1}, \ldots, G_{n}\right\}$, and $m \in\{1, \ldots, n\}$. Let $\left(\tau_{m+1}, \ldots, \tau_{n}\right)$ be the least solution of the extended algebraic tree system $\mathfrak{S}^{\prime}=\left(\Phi^{\prime}, Z, \Sigma, E^{\prime}\right)$, where $\Phi^{\prime}=\left\{G_{m+1}, \ldots, G_{n}\right\}$ and $E^{\prime}=\left\{G_{i}\left(z_{1}, \ldots, z_{r_{i}}\right)=\right.$ $\left.s_{i}\left(z_{1}, \ldots, z_{r_{i}}\right) \mid m+1 \leq i \leq n\right\}$. Hence, $\tau_{j} \in A\left\langle\left\langle T_{\Sigma \cup\left\{G_{1}, \ldots, G_{m}\right\}}\left(X \cup Z_{r_{j}}\right)\right\rangle\right.$, $m+1 \leq i \leq n$. Furthermore, let $\left(\tau_{1}, \ldots, \tau_{m}\right)$ be the least solution of the extended algebraic system $\mathfrak{S}^{\prime \prime}=\left(\Phi^{\prime \prime}, Z, \Sigma, E^{\prime \prime}\right)$, where $\Phi^{\prime \prime}=\left\{G_{1}, \ldots, G_{m}\right\}$ and $E^{\prime \prime}=\left\{G_{i}\left(z_{1}, \ldots, z_{r_{i}}\right)=s_{i}\left(z_{1}, \ldots, z_{r_{i}}\right)\left[\tau_{m+1} / G_{m+1}, \ldots, \tau_{n} / G_{n}\right] \mid 1 \leq i \leq m\right\}$. Then

$$
\left(\tau_{1}, \ldots, \tau_{m}, \tau_{m+1}\left[\tau_{1} / G_{1}, \ldots, \tau_{m} / G_{m}\right], \ldots, \tau_{n}\left[\tau_{1} / G_{1}, \ldots, \tau_{m} / G_{m}\right]\right)
$$

is the least solution of the original extended algebraic tree system.

We now proceed analogously to Section 4.

Theorem 5.13 $\left\langle A^{\text {alg }}\left\langle\left\langle T_{\Sigma \cup \Phi_{\infty}}(X \cup Z)\right\rangle\right\rangle,+, \cup, \bar{\Sigma} \cup \bar{\Phi}_{\infty}\right\rangle$ is a distributive $\Sigma \cup \Phi_{\infty}$ algebra closed under scalar product that contains $A\left\langle T_{\Sigma \cup \Phi_{\infty}}(X \cup Z)\right\rangle$. 
Hence, for $G \in \Phi_{\infty}$ of rank $r$ and $\sigma_{1}, \ldots, \sigma_{r} \in A^{\operatorname{alg}}\left\langle\left\langle T_{\Sigma \cup \Phi_{\infty}}(X \cup Z)\right\rangle\right\rangle$, $\bar{G}\left(\sigma_{1}, \ldots, \sigma_{r}\right)$ is again in $A^{\operatorname{alg}}\left\langle\left\langle T_{\Sigma \cup \Phi_{\infty}}(X \cup Z)\right\rangle\right\rangle$.

Proof. We only prove the second sentence. The proof of the first sentence is analogous to the proof of Theorem 4.1.

Let $\sigma_{1}, \ldots, \sigma_{r} \in A\left\langle\left\langle T_{\Sigma \cup\left\{G_{k_{1}}, \ldots, G_{k_{m}}\right\}}\left(X \cup\left\{z_{1}, \ldots, z_{k}\right\}\right)\right\rangle\right\rangle$. Then there exist $r$ algebraic tree systems $G_{t j}\left(z_{1}, \ldots, z_{i_{t j}}\right)=s_{t j}, 1 \leq t \leq r, 1 \leq j \leq n_{r}$, where the rank of $G_{t 1}$ is $k$, such that the first components of their least solutions are $\sigma_{t}$.

Consider now the algebraic tree system

$$
\begin{aligned}
& H\left(z_{1}, \ldots, z_{k}\right)=G\left(G_{11}\left(z_{1}, \ldots, z_{k}\right), \ldots, G_{r 1}\left(z_{1}, \ldots, z_{k}\right)\right) \\
& G_{t j}\left(z_{1}, \ldots, z_{i_{t j}}\right)=s_{t j}, \quad 1 \leq t \leq r, 1 \leq j \leq n_{r}
\end{aligned}
$$

By the Bekić-De Bakker-Scott rule, the $H$-component of its least solution is then given by $\bar{G}\left(\sigma_{1}, \ldots, \sigma_{r}\right)$.

A distributive $\Sigma \cup \Phi_{\infty}$-algebra $\left\langle V,+, 0, \bar{\Sigma} \cup \bar{\Phi}_{\infty}\right\rangle, V \subseteq A\left\langle\left\langle T_{\Sigma \cup \Phi_{\infty}}(X \cup Z)\right\rangle\right\rangle$ is called equationally closed iff $V$ is closed under scalar product, and for all $s \in V$ and $G \in \Phi_{\infty}$ the formal tree series $\mu G . s$ is again in $V$. Here $\mu G . s$ denotes the least solution of $G\left(z_{1}, \ldots, z_{r}\right)=s$, where $r$ is the rank of $G$. By definition, $A^{\text {equ }}\left\langle\left\langle T_{\Sigma \cup \Phi_{\infty}}(X \cup Z)\right\rangle\right\rangle$ is the least equationally closed distributive $\Sigma \cup \Phi_{\infty}$-algebra containing $A\left\langle T_{\Sigma \cup \Phi_{\infty}}(X \cup Z)\right\rangle$. Observe that each power series in $A^{\text {equ }}\left\langle\left\langle T_{\Sigma \cup \Phi_{\infty}}(X \cup Z)\right\rangle\right\rangle$ is in fact a power series in $A\left\langle\left\langle T_{\Sigma \cup \Phi}\left(X \cup Z_{r}\right)\right\rangle\right\rangle$ for some finite $\Phi \subset \Phi_{\infty}$ and $r \geq 0$.

We will prove that $A^{\mathrm{equ}}\left\langle\left\langle T_{\Sigma \cup \Phi_{\infty}}(X \cup Z)\right\rangle\right\rangle=A^{\mathrm{alg}}\left\langle\left\langle T_{\Sigma \cup \Phi_{\infty}}(X \cup Z)\right\rangle\right\rangle$. We first show that $A^{\mathrm{equ}}\left\langle\left\langle T_{\Sigma \cup \Phi_{\infty}}(X \cup Z)\right\rangle\right\rangle$ is closed under substitution for function variables.

Theorem 5.14 Consider tree series $s$ and $\sigma_{j}, 1 \leq j \leq n$, in $A^{\mathrm{equ}}\left\langle\left\langle T_{\Sigma \cup \Phi_{\infty}}(X \cup\right.\right.$ $Z)\rangle\rangle$ and assume that $s\left(z_{1}, \ldots, z_{r}, G_{i_{1}}, \ldots, G_{i_{n}}\right) \in A\left\langle\left\langle T_{\hat{\Sigma} \cup\left\{G_{i_{1}}, \ldots, G_{i_{n}}\right\}}\left(X \cup Z_{r}\right)\right\rangle\right\rangle$ and $\sigma_{j} \in A\left\langle\left\langle T_{\hat{\Sigma}}\left(X \cup Z_{r_{i_{j}}}\right)\right\rangle\right\rangle$, where $\hat{\Sigma}=\Sigma \cup\left\{G_{k_{1}}, \ldots, G_{k_{m}}\right\}$ and $i_{1}, \ldots, i_{n}, k_{1}, \ldots, k_{m} \geq$ 0 are mutually disjoint.

Then $\bar{s}\left(z_{1}, \ldots, z_{r}, \sigma_{1}, \ldots, \sigma_{n}\right)$ is again in $A^{\mathrm{equ}}\left\langle\left\langle T_{\Sigma \cup \Phi_{\infty}}(X \cup Z)\right\rangle\right\rangle$.

Proof. The proof is by induction on the number of applications of the operations $\bar{\omega} \in \bar{\Sigma}, \bar{G} \in \bar{\Phi}_{\infty}$, sum, scalar product and $\mu$ to generate $s\left(z_{1}, \ldots, z_{r}, G_{i_{1}}, \ldots, G_{i_{n}}\right)$ from polynomials.

(i) Let $s\left(z_{1}, \ldots, z_{r}, G_{i_{1}}, \ldots, G_{i_{n}}\right) \in A\left\langle T_{\hat{\Sigma} \cup\left\{G_{i_{1}}, \ldots, G_{i_{n}}\right\}}\left(X \cup Z_{r}\right)\right\rangle$. Since $\bar{s}\left(z_{1}, \ldots, z_{r}, \sigma_{1}, \ldots, \sigma_{n}\right)$ is generated from $\sigma_{1}, \ldots, \sigma_{n}$ and $z_{1}, \ldots, z_{r}$ by application of sum, $\bar{\omega} \in \bar{\Sigma}$, $\bar{G}_{k_{1}}, \ldots, \bar{G}_{k_{m}}$, and scalar product, we infer that $\bar{s}\left(z_{1}, \ldots, z_{r}, \sigma_{1}, \ldots, \sigma_{n}\right) \in A^{\text {equ }}\left\langle\left\langle T_{\Sigma \cup \Phi_{\infty}}(X \cup\right.\right.$ $Z)\rangle$.

(ii) We only prove the case of the operator $\mu$. Choose a $G \in \Phi_{\infty}$ with rank $r$ that is unequal to $G_{i_{1}}, \ldots, G_{i_{n}}, G_{k_{1}}, \ldots, G_{k_{m}}$. Without loss of generality we assume that $s\left(z_{1}, \ldots, z_{r}, G_{i_{1}}, \ldots, G_{i_{n}}\right)=\mu G . s^{\prime}\left(z_{1}, \ldots, z_{r}, G_{i_{1}}, \ldots, G_{i_{n}}, G\right)$. By induction hypothesis, we have that $s^{\prime}\left(z_{1}, \ldots, z_{r}, G_{i_{1}}, \ldots, G_{i_{n}}, G\right)$ is in $A^{\text {equ }}\left\langle\left\langle T_{\Sigma \cup \Phi_{\infty}}(X \cup\right.\right.$ 
$Z)\rangle\rangle$. Hence $\bar{s}\left(z_{1}, \ldots, z_{r}, \sigma_{1}, \ldots, \sigma_{n}\right)=\mu G \cdot \bar{s}^{\prime}\left(z_{1}, \ldots, z_{r}, \sigma_{1}, \ldots, \sigma_{n}, G\right)$ is in $A^{\text {equ }}\left\langle\left\langle T_{\Sigma \cup \Phi_{\infty}}(X \cup\right.\right.$ $Z)\rangle$ by the parameter identity.

Theorem 5.15 (Bozapalidis [11], Section 6.) $A^{\text {equ }}\left\langle\left\langle T_{\Sigma \cup \Phi_{\infty}}(X \cup Z)\right\rangle\right\rangle=A^{\text {alg }}\left\langle\left\langle T_{\Sigma \cup \Phi_{\infty}}(X \cup\right.\right.$ $Z)\rangle$

Proof. We show that $A^{\text {alg }}\left\langle\left\langle T_{\Sigma \cup \Phi_{\infty}}(X \cup Z)\right\rangle \subseteq \subseteq A^{\text {equ }}\left\langle\left\langle T_{\Sigma \cup \Phi_{\infty}}(X \cup Z)\right\rangle\right\rangle\right.$. The proof is by induction on the number of variables of algebraic systems. We use the following induction hypothesis:

If $\left(\tau_{1}, \ldots, \tau_{n}\right), \tau_{j} \in A^{\mathrm{alg}}\left\langle\left\langle T_{\Sigma \cup \Phi_{\infty}}(X \cup Z)\right\rangle\right\rangle, 1 \leq j \leq n$, is the least solution of an algebraic system $G_{j}\left(z_{1}, \ldots, z_{r_{j}}\right)=s_{j}\left(z_{1}, \ldots, z_{r_{j}}, G_{1}, \ldots, G_{n}\right), 1 \leq j \leq$ $n$, with $n$ function variables $G_{1}, \ldots, G_{n}$, where $s_{j}\left(z_{1}, \ldots, z_{r_{j}}, G_{1}, \ldots, G_{n}\right) \in$ $A\left\langle T_{\Sigma \cup \Phi_{\infty}}(X \cup Z)\right\rangle$ then $\tau_{j} \in A^{\text {equ }}\left\langle\left\langle T_{\Sigma \cup \Phi_{\infty}}(X \cup Z)\right\rangle\right\rangle_{\text {. }}$

(i) Let $n=1$ and assume that $s \in A^{\text {alg }}\left\langle\left\langle T_{\Sigma \cup \Phi_{\infty}}(X \cup Z)\right\rangle\right\rangle$ is the least solution of the algebraic system $G_{1}\left(z_{1}, \ldots, z_{r_{1}}\right)=p\left(z_{1}, \ldots, z_{r_{1}}, G_{1}\right)$. Since $p\left(z_{1}, \ldots, z_{r_{1}}, G_{1}\right)$ is a polynomial, $s=\mu G_{1} . p\left(z_{1}, \ldots, z_{r_{1}}, G_{1}\right) \in A^{\text {equ }}\left\langle\left\langle T_{\Sigma \cup \Phi_{\infty}}(X \cup\right.\right.$ $Z)\rangle$.

Consider the algebraic system $G_{j}\left(z_{1}, \ldots, z_{r_{j}}\right)=s_{j}\left(z_{1}, \ldots, z_{r_{j}}, G_{1}, \ldots, G_{n+1}\right)$, $1 \leq j \leq n+1, n \geq 1$. Let $\left(\tau_{2}\left(G_{1}\right), \ldots, \tau_{n+1}\left(G_{1}\right)\right), \tau_{j}\left(G_{1}\right) \in A^{\text {alg }}\left\langle\left\langle T_{\Sigma \cup \Phi_{\infty}}(X \cup\right.\right.$ $Z)\rangle, 2 \leq j \leq n+1$, be the least solution of the algebraic system $G_{j}\left(z_{1}, \ldots, z_{r_{j}}\right)=$ $s_{j}\left(z_{1}, \ldots, z_{r_{j}}, G_{1}, \ldots, G_{n+1}\right), 2 \leq j \leq n+1$. By our induction hypothesis we infer that $\tau_{j}\left(G_{1}\right) \in A^{\text {equ }}\left\langle\left\langle T_{\Sigma \cup \Phi_{\infty}}(X \cup Z)\right\rangle\right\rangle$. Hence, by Theorem 5.14, $p\left(G_{1}\right)=$ $\bar{s}_{1}\left(z_{1}, \ldots, z_{r_{1}}, G_{1}, \tau_{2}\left(G_{1}\right), \ldots, \tau_{n+1}\left(G_{1}\right)\right)$ is in $A^{\text {equ }}\left\langle\left\langle T_{\Sigma \cup \Phi_{\infty}}(X \cup Z)\right\rangle\right.$. This implies that $\mu G_{1} \cdot p\left(G_{1}\right)$ is in $A^{\text {equ }}\left\langle\left\langle T_{\Sigma \cup \Phi_{\infty}}(X \cup Z)\right\rangle\right.$. Again by Theorem 5.14 $\bar{\tau}_{j}\left(\mu G_{1} . p\left(G_{1}\right)\right) \in A^{\text {equ }}\left\langle\left\langle T_{\Sigma \cup \Phi_{\infty}}(X \cup Z)\right\rangle\right\rangle, 2 \leq j \leq n+1$. By the Bekić-De BakkerScott rule,

$$
\left(\mu G_{1} \cdot p\left(G_{1}\right), \bar{\tau}_{2}\left(\mu G_{1} \cdot p\left(G_{1}\right)\right), \ldots, \bar{\tau}_{n+1}\left(\mu G_{1} \cdot p\left(G_{1}\right)\right)\right)
$$

is the least solution of the algebraic system $G_{j}\left(z_{1}, \ldots, z_{r_{j}}\right)=s_{j}\left(z_{1}, \ldots, z_{r_{j}}, G_{1}, \ldots, G_{n+1}\right)$, $1 \leq j \leq n+1$. Hence, the components of the least solution of this algebraic system are in $A^{\text {equ }}\left\langle\left\langle T_{\Sigma \cup \Phi_{\infty}}(X \cup Z)\right\rangle\right\rangle$.

(ii) We show that $A^{\text {alg }}\left\langle\left\langle T_{\Sigma \cup \Phi_{\infty}}(X \cup Z)\right\rangle\right\rangle$ is an equationally closed distributive $\Sigma \cup \Phi_{\infty}$-algebra that contains $A\left\langle T_{\Sigma}(X \cup Z\rangle\right.$. This will imply $A^{\text {equ }}\left\langle\left\langle T_{\Sigma \cup \Phi_{\infty}}(X \cup\right.\right.$ $Z)\rangle \subseteq A^{\text {alg }}\left\langle\left\langle T_{\Sigma \cup \Phi_{\infty}}(X \cup Z)\right\rangle\right\rangle$. By Theorem 5.13, we have only to show that $\mu G . s$, $s \in A^{\text {alg }}\left\langle\left\langle T_{\Sigma \cup \Phi_{\infty}}(X \cup Z)\right\rangle\right\rangle$ is in $A^{\text {alg }}\left\langle\left\langle T_{\Sigma \cup \Phi_{\infty}}(X \cup Z)\right\rangle\right.$. Let $\left(\tau_{2}\left(G_{1}\right), \ldots, \tau_{n+1}\left(G_{1}\right)\right)$ be the least solution of the algebraic system $G_{j}\left(z_{1}, \ldots, z_{r_{j}}\right)=s_{j}\left(z_{1}, \ldots, z_{r_{j}}, G_{1}, \ldots, G_{n+1}\right)$, $2 \leq j \leq n+1$, where $G_{1}$ is of rank $r_{2}$ and take $s=\tau_{2}$. Consider now the algebraic system $G_{1}\left(z_{1}, \ldots, z_{r_{2}}\right)=s_{2}\left(z_{1}, \ldots, z_{r_{2}}, G_{1}, \ldots, G_{n+1}\right), G_{j}\left(z_{1}, \ldots, z_{r_{j}}\right)=$ $s_{j}\left(z_{1}, \ldots, z_{r_{j}}, G_{1}, \ldots, G_{n+1}\right), 2 \leq j \leq n+1$. Then, by the Bekić-De BakkerScott rule, $\mu G_{1} \cdot \bar{s}_{2}\left(z_{1}, \ldots, z_{r_{2}}, G_{1}, \tau_{2}\left(G_{1}\right), \ldots, \tau_{n+1}\left(G_{1}\right)\right)=\mu G_{1} \cdot \tau_{2}\left(G_{1}\right)$ is the first component of its least solution.

We now introduce algebraic tree series expressions. Assume that $A, \Sigma, X, Z, \Phi_{\infty}$ and $U=\{+, \cdot, \mu,[]$,$\} are mutually disjoint. A word E$ over $A \cup \Sigma \cup X \cup Z \cup \Phi_{\infty} \cup U$ is an algebraic tree series expression over $\left(A, \Sigma, X, Z, \Phi_{\infty}\right)$ iff 
(i) $E$ is in $X \cup Z$, or

(ii) $E$ is of one of the forms $\left[E_{1}+E_{2}\right], \omega\left(E_{1}, \ldots, E_{k}\right), G\left(E_{1}, \ldots, E_{k}\right), a E_{1}$, or $\mu G . E_{1}$, where $E_{1}, \ldots, E_{k}$ are algebraic tree series expressions over $\left(A, \Sigma, X, Z, \Phi_{\infty}\right)$, for $\omega \in \Sigma$ of rank $k, G \in \Phi_{\infty}$ of rank $k, k \geq 0$, and $a \in A$.

Each algebraic tree series expression $E$ over $\left(A, \Sigma, X, Z, \Phi_{\infty}\right)$ denotes a formal tree series $|E|$ in $A\left\langle\left\langle T_{\Sigma \cup \Phi_{\infty}}(X \cup Z)\right\rangle\right\rangle$ according to the followin conventions:

(i) If $E$ is in $X \cup Z$ then $E$ denotes the tree series $E$, i. e., $|E|=E$.

(ii) For algebraic tree series expressions $E_{1}, \ldots, E_{k}$ over $\left(A, \Sigma, X, Z, \Phi_{\infty}\right), \omega \in$ $\Sigma$ of rank $k, G \in \Phi_{\infty}$ of rank $k, k \geq 0, a \in A$, we define

$$
\begin{aligned}
& \left|\left[E_{1}+E_{2}\right]\right|=\left|E_{1}\right|+\left|E_{2}\right|, \\
& \left|\omega\left(E_{1}, \ldots, E_{k}\right)\right|=\bar{\omega}\left(\left|E_{1}\right|, \ldots,\left|E_{k}\right|\right), \\
& \left|G\left(E_{1}, \ldots, E_{k}\right)\right|=\bar{G}\left(\left|E_{1}\right|, \ldots,\left|E_{k}\right|\right), \\
& \left|a E_{1}\right|=a\left|E_{1}\right| \\
& \left|\mu G \cdot E_{1}\right|=\mu G \cdot\left|E_{1}\right|
\end{aligned}
$$

Let $\Phi_{1}, \Phi_{2}, \Phi_{3}$ be mappings from the set of algebraic tree series expressions over $\left(A, \Sigma, X, Z, \Phi_{\infty}\right)$ into the set of finite subsets of $X \cup Z \cup \Phi_{\infty}$ defined by

(i) $\Phi_{1}(x)=\emptyset, \Phi_{2}(x)=\{x\}, \Phi_{3}(x)=\emptyset, x \in X$, $\Phi_{1}(z)=\{z\}, \Phi_{2}(z)=\emptyset, \Phi_{3}(z)=\emptyset, z \in Z$.

(ii) $\Phi_{j}\left(\left[E_{1}+E_{2}\right]\right)=\Phi_{j}\left(E_{1}\right)+\Phi_{j}\left(E_{2}\right), j=1,2,3$,

$$
\Phi_{j}\left(\omega\left(E_{1}, \ldots, E_{k}\right)\right)=\Phi_{j}\left(E_{1}\right) \cup \ldots \cup \Phi_{j}\left(E_{k}\right), j=1,2,3,
$$

$\Phi_{j}\left(G\left(E_{1}, \ldots, E_{k}\right)\right)=\Phi_{j}\left(E_{1}\right) \cup \ldots \cup \Phi_{j}\left(E_{k}\right), j=1,2$,

$\Phi_{3}\left(G\left(E_{1}, \ldots, E_{k}\right)\right)=\Phi_{3}\left(E_{1}\right) \cup \ldots \cup \Phi_{3}\left(E_{k}\right) \cup\{G\}$,

$\Phi_{j}\left(a E_{1}\right)=\Phi_{j}\left(E_{1}\right) j=1,2,3$,

$\Phi_{j}\left(\mu G \cdot E_{1}\right)=\Phi_{j}\left(E_{1}\right)-\{G\} j=1,2,3$,

for algebraic tree series expressions $E_{1}, \ldots, E_{k}$ over $\left(A, \Sigma, X, Z, \Phi_{\infty}\right), \omega \in$ $\Sigma$ of rank $k, G \in \Phi_{\infty}$ of rank $k, k \geq 0, a \in A$.

Given an algebraic tree series expression $E$ over $\left(A, \Sigma, X, Z, \Phi_{\infty}\right), \Phi_{1}(E) \subseteq Z$ contains the variables of $E, \Phi_{2}(E) \subseteq X$ contains the used symbols of the leaf alphabet $X$ and $\Phi_{3}(E) \subseteq G$ contains the "free function variables" of $E$. This means that $|E|$ is a formal tree series in $A\left\langle\left\langle T_{\Sigma \cup \Phi_{3}(E)}\left(\Phi_{2}(E) \cup \Phi_{1}(E)\right)\right\rangle\right\rangle$.

Theorem 5.15 and the above definitions yiel some corollaries.

Corollary 5.16 A tree series $s$ is in $A^{\text {equ }}\left\langle\left\langle T_{\Sigma \cup \Phi_{\infty}}(X \cup Z)\right\rangle\right\rangle \cap A\left\langle\left\langle T_{\Sigma \cup \Phi_{3}(E)}\left(\Phi_{2}(E) \cup\right.\right.\right.$ $\left.\left.\left.\Phi_{1}(E)\right)\right\rangle\right\rangle$ iff there is an algebraic tree series expression $E$ over $\left(A, \Sigma, X, Z, \Phi_{\infty}\right)$ such that $s=|E|$. 
Corollary $5.17 A$ tree series $s$ is in $A^{\text {equ }}\left\langle\left\langle T_{\Sigma \cup \Phi_{\infty}}(X \cup Z)\right\rangle\right\rangle \cap A\left\langle\left\langle T_{\Sigma}\left(\Phi_{2}(E)\right)\right\rangle\right\rangle$ iff there is an algebraic tree series expression $E$ over $\left(A, \Sigma, X, Z, \Phi_{\infty}\right)$ such that $s=|E|$, where $\Phi_{1}(E)=\Phi_{3}(E)=\emptyset$.

Corollary 5.18 A tree series is in $A^{\text {alg }}\left\langle\left\langle T_{\Sigma}\left(\Phi_{2}(E)\right)\right\rangle\right\rangle$ iff there exists an algebraic tree series expression $E$ over $\left(A, \Sigma, X, Z, \Phi_{\infty}\right)$ such that $s=|E|$, where $\Phi_{1}(E)=\Phi_{3}(E)=\emptyset$.

We summarize our results in a Kleene-like theorem.

Theorem 5.19 The following statements on a power series $r \in A\left\langle\left\langle T_{\Sigma}(X)\right\rangle\right\rangle$ are equivalent.

(i) $r$ is an algebraic tree series,

(ii) $r$ is the behavior of a simple pushdown tree automaton,

(iii) $r$ is the behavior of a simple restricted pushdown tree automaton,

(iv) there exists an algebraic tree series expression $E$ over $\left(A, \Sigma, X, Z, \Phi_{\infty}\right)$, where $\Phi_{1}(E)=\Phi_{3}(E)=\emptyset$, such that $r=|E|$.

If we interprete algebraic tree series expressions in $\mathbb{B}\left\langle\left\langle T_{\Sigma \cup \Phi_{\infty}}(X \cup Z)\right\rangle\right\rangle$ then we get analogous results on formal tree languages.

Example 5.2. Consider the algebraic tree system $\mathfrak{S}=\left(\Phi, Z, \Sigma, E, G_{0}\right)$ with initial function variable $G_{0}$, specified by $\Phi=\Phi_{0} \cup \Phi_{2}, \Phi_{0}=\left\{G_{0}\right\}, \Phi_{2}=$ $\{G\}, \Sigma=\Sigma_{2}=\{b\}, X=\left\{c_{1}, c_{2}\right\}$, and $E=\left\{G_{0}=G\left(c_{1}, c_{2}\right), G\left(z_{1}, z_{2}\right)=\right.$ $\left.G\left(b\left(z_{1}, z_{1}\right), b\left(z_{2}, z_{2}\right)\right)+b\left(z_{1}, z_{2}\right)\right\}$. (This algebraic system is a simplified version of that in Example 5.1.) The initial component of its least solution is given by

$$
\left|\mu G \cdot\left[G\left(b\left(c_{1}, c_{1}\right), b\left(c_{2}, c_{2}\right)\right)+b\left(c_{1}, c_{2}\right)\right]\right|=\sum_{j \geq 0} b\left(t_{1}^{j}, t_{2}^{j}\right),
$$

where $t_{i}^{0}=c_{i}, t_{i}^{j+1}=b\left(t_{i}^{j}, t_{i}^{j}\right), i=1,2, j \geq 0$.

\section{Tree series transducers}

Tree transducers have been introduced in Rounds [50, 52] and Thatcher [56, 57]. Kuich [39] generalized a restricted form of top-down tree transducers to tree series transducers which map formal tree series into formal tree series. Engelfriet, Fülöp, Vogler [19] and Fülöp, Vogler [23] generalized this approach and defined bottom-up and top-down tree series transducers as generalization of frontier-to-root and root-to-frontier tree transducers in the sense of Gécseg, Steinby $[24,25]$.

In this section we only consider the case of top-down tree series transducers. (The bottom-up tree series transducers use a generalization of IO-substitutions 
and are difficult to handle.) Our definition of top-down tree series transducers is different but equivalent to the definition of Engelfriet, Fülöp, Vogler [23].

We then define linear nondeleting recognizable tree series transducers and show that they preserve recognizability of tree series.

We define

$$
\left(A\left\langle\left\langle T_{\Sigma}(X)\right\rangle\right\rangle\right)^{I_{1} \times I_{2}^{*}}=\bigcup_{m \geq 0}\left(A\left\langle\left\langle T_{\Sigma}(X)\right\rangle\right\rangle\right)^{I_{1} \times I_{2}^{m}} .
$$

A tree representation $\mu$ (with state set $Q$, ranked input alphabet $\Sigma$, input leaf alphabet $X$, ranked output alphabet $\Sigma^{\prime}$, output leaf alphabet $X^{\prime}$, over the semiring $A)$ is a family $\mu=\left(\mu_{k} \mid k \geq 0\right)$ of mappings

$$
\begin{aligned}
& \mu_{k}: \Sigma_{k} \rightarrow\left(A\left\langle\left\langle T_{\Sigma^{\prime}}\left(X^{\prime} \cup Y\right)\right\rangle\right\rangle\right)^{Q \times\left(Q \times Z_{k}\right)^{*}}, k \geq 1, \\
& \mu_{0}: \Sigma_{0} \cup X \rightarrow\left(A\left\langle\left\langle T_{\Sigma^{\prime}}\left(X^{\prime}\right)\right\rangle\right\rangle\right)^{Q \times 1},
\end{aligned}
$$

such that, if $\mu_{k}(\omega) \in\left(A\left\langle\left\langle T_{\Sigma^{\prime}}\left(X^{\prime} \cup Y\right)\right\rangle\right\rangle\right)^{Q \times\left(Q \times Z_{k}\right)^{m}}$ for some $m \geq 0$ and $\omega \in \Sigma_{k}$, $k \geq 1$, then $\mu_{k}(\omega) \in\left(A\left\langle\left\langle T_{\Sigma^{\prime}}\left(X^{\prime} \cup Y_{m}\right)\right\rangle\right\rangle\right)^{Q \times\left(Q \times Z_{k}\right)^{m}}$. Observe that $\mu_{k}(\omega)$, $\omega \in \Sigma_{k}, k \geq 1$, induces a mapping

$$
\mu_{k}(\omega):\left(A\left\langle\left\langle T_{\Sigma}\left(X^{\prime}\right)\right\rangle\right\rangle\right)^{Q \times 1} \times \cdots \times\left(A\left\langle\left\langle T_{\Sigma}\left(X^{\prime}\right)\right\rangle\right\rangle\right)^{Q \times 1} \rightarrow\left(A\left\langle\left\langle T_{\Sigma}\left(X^{\prime}\right)\right\rangle\right\rangle\right)^{Q \times 1}
$$

(there are $k$ argument vectors; see the definition before Theorem 2.13).

Since $\left\langle\left(A\left\langle\left\langle T_{\Sigma^{\prime}}\left(X^{\prime}\right)\right\rangle\right\rangle\right)^{Q \times 1},\left(\mu_{k}(\omega) \mid \omega \in \Sigma_{k}, k \geq 0\right)\right\rangle$ is a $\Sigma$-algebra, the mapping

$$
\mu_{0}: X \rightarrow\left(A\left\langle\left\langle T_{\Sigma^{\prime}}\left(X^{\prime}\right)\right\rangle\right\rangle\right)^{Q \times 1}
$$

can be uniquely extended to a morphism

$$
\mu: T_{\Sigma}(X) \rightarrow\left(A\left\langle\left\langle T_{\Sigma^{\prime}}\left(X^{\prime}\right)\right\rangle\right\rangle\right)^{Q \times 1}
$$

by

for $\omega \in \Sigma_{k}, t_{1}, \ldots, t_{k} \in T_{\Sigma}(X), k \geq 0$.

One more extension yields

$$
\mu: A\left\langle\left\langle T_{\Sigma}(X)\right\rangle\right\rangle \rightarrow\left(A\left\langle\left\langle T_{\Sigma^{\prime}}\left(X^{\prime}\right)\right\rangle\right\rangle\right)^{Q \times 1}
$$

by

$$
\mu(s)=\sum_{t \in T_{\Sigma}(X)}(s, t) \otimes \mu(t), \quad s \in A\left\langle\left\langle T_{\Sigma}(X)\right\rangle\right\rangle,
$$

where $\otimes$ denotes the Kronecker product. (See Kuich, Salomaa [45], Section 4.) In our case this means that each entry of $\mu(t)$ is multiplied by $(s, t)$. Hence, for $q \in Q$,

$$
\mu(s)_{q}=\sum_{t \in T_{\Sigma}(X)}(s, t) \mu(t)_{q}, \quad s \in A\left\langle\left\langle T_{\Sigma}(X)\right\rangle\right\rangle .
$$


We have denoted a tree representation $\mu$ and the mapping $\mu: A\left\langle\left\langle T_{\Sigma}(X)\right\rangle\right\rangle \rightarrow$ $\left(A\left\langle\left\langle T_{\Sigma^{\prime}}\left(X^{\prime}\right)\right\rangle\right\rangle\right)^{Q \times 1}$ induced by it by the same letter $\mu$. This should not lead to any confusion.

A (top-down) tree series transducer (with state set $Q$, ranked input alphabet $\Sigma$, input leaf alphabet $X$, ranked output alphabet $\Sigma^{\prime}$, output leaf alphabet $X^{\prime}$, over the semiring $A$ )

$$
\mathfrak{T}=(Q, \mu, S)
$$

is given by

(i) a nonempty finite set $Q$ of states,

(ii) a tree representation $\mu$ with $Q, \Sigma, X, \Sigma^{\prime}, X^{\prime}$ over $A$,

(iii) an initial state vector $S \in\left(A\left\langle\left\langle T_{\Sigma^{\prime}}\left(Z_{1}\right)\right\rangle\right\rangle\right)^{1 \times Q}$, where $S_{q}=a_{q} z_{1}, a_{q} \in A$, $q \in Q$.

The mapping

$$
\|\mathfrak{T}\|: A\left\langle\left\langle T_{\Sigma}(X)\right\rangle\right\rangle \rightarrow A\left\langle\left\langle T_{\Sigma^{\prime}}\left(X^{\prime}\right)\right\rangle\right\rangle
$$

realized by a tree series transducer $\mathfrak{T}=(Q, \mu, S)$ is defined by

$$
\begin{aligned}
& \|\mathfrak{T}\|(s)=S(\mu(s))=\sum_{q \in Q}\left(S_{q}, z_{1}\right) \mu(s)_{q}= \\
& \sum_{q \in Q} \sum_{t \in T_{\Sigma}(X)} a_{q}(s, t) \mu(t)_{q}, \quad s \in A\left\langle\left\langle T_{\Sigma}(X)\right\rangle\right\rangle .
\end{aligned}
$$

A tree representation $\mu$ is called polynomial iff the entries of the images of $\mu_{k}, k \geq 0$, are polynomials. A tree series transducer $\mathfrak{T}=(Q, \mu, S)$ is called polynomial iff $\mu$ is a polynomial tree representation.

Example 6.1. (See Example IV.1.6 of Gécseg, Steinby [24].) Let $Q=\left\{a_{0}, a_{1}, a_{2}\right\}$, $\Sigma=\Sigma_{1}=\{\sigma\}, X=\{x\}, \Sigma^{\prime}=\Sigma_{1}^{\prime} \cup \Sigma_{2}^{\prime}, \Sigma_{1}^{\prime}=\left\{\omega_{1}\right\}, \Sigma_{2}^{\prime}=\left\{\omega_{2}\right\}, X^{\prime}=\left\{x_{1}^{\prime}, x_{2}^{\prime}\right\}$.

The nonnull entries of $\mu_{0}$ and $\mu_{1}$ are given by

$$
\begin{aligned}
& \mu_{0}(x)_{a_{1}}=x_{1}^{\prime}, \quad \mu_{0}(x)_{a_{2}}=x_{2}^{\prime}, \\
& \mu_{1}(\sigma)_{a_{0},\left(\left(a_{1}, z_{1}\right),\left(a_{2}, z_{1}\right)\right)}=\omega_{2}\left(y_{1}, y_{2}\right), \\
& \mu_{1}(\sigma)_{a_{1},\left(a_{1}, z_{1}\right)}=\omega_{1}\left(y_{1}\right), \quad \mu_{1}(\sigma)_{a_{2},\left(a_{2}, z_{1}\right)}=\omega_{1}\left(y_{1}\right) .
\end{aligned}
$$

Let $S=\left(z_{1}, 0,0\right)$ and consider the tree series transducer $\mathfrak{T}=\left(Q,\left(\mu_{0}, \mu_{1}\right), S\right)$. We claim that, for $n \geq 0$,

$$
\mu\left(\sigma^{n}(x)\right)_{a_{i}}=\omega_{1}^{n}\left(x_{i}^{\prime}\right), \quad i=1,2,
$$

and prove it by induction on $n$.

We have

$$
\mu(x)_{a_{i}}=\mu_{0}(x)_{a_{i}}=x_{i}^{\prime},
$$

and, for $n>0$,

$$
\begin{aligned}
& \mu\left(\sigma^{n}(x)\right)_{a_{i}}=\mu_{1}(\sigma)\left[\mu\left(\sigma^{n-1}(x)\right)\right]_{a_{i}}= \\
& \mu_{1}(\sigma)_{a_{i},\left(a_{i}, z_{1}\right)}\left[\omega_{1}^{n-1}\left(x_{i}^{\prime}\right)\right]= \\
& \omega_{1}\left(y_{1}\right)\left(\omega_{1}^{n-1}\left(x_{i}^{\prime}\right)\right)=\omega_{1}^{n}\left(x_{i}^{\prime}\right), \quad i=1,2 .
\end{aligned}
$$


Hence, we obtain for $n \geq 1$,

$$
\begin{aligned}
& \|\mathfrak{T}\|\left(\sigma^{n}(x)\right)=\mu\left(\sigma^{n}(x)\right)_{a_{0}}= \\
& \mu_{1}(\sigma)\left[\mu\left(\sigma^{n-1}(x)\right)\right]_{a_{0}}= \\
& \mu_{1}(\sigma)_{a_{0},\left(\left(a_{1}, z_{1}\right),\left(a_{2}, z_{1}\right)\right)}\left(\mu\left(\sigma^{n-1}(x)\right)_{a_{1}}, \mu\left(\sigma^{n-1}(x)\right)_{a_{2}}\right)= \\
& \omega_{2}\left(\omega_{1}^{n-1}\left(x_{1}^{\prime}\right), \omega_{1}^{n-1}\left(x_{2}^{\prime}\right)\right) .
\end{aligned}
$$

Given a formal tree series

$$
s=(s, x) x+\sum_{n \geq 1}\left(s, \sigma^{n}(x)\right) \sigma^{n}(x),
$$

we obtain

$$
\|\mathfrak{T}\|(s)=\sum_{n \geq 1}\left(s, \sigma^{n}(x)\right) \omega_{2}\left(\omega_{1}^{n-1}\left(x_{1}^{\prime}\right), \omega_{1}^{n-1}\left(x_{2}^{\prime}\right)\right) .
$$

In connection with Example IV.1.6 of Gécseg, Steinby [24], Example 6.1 gives also an intuitive feeling, how a root-to-frontier tree transducer in the sense of Gécseg, Steinby [24] is simulated by a top-down tree series transducer over the semiring $\mathbb{B}$. Formally, we have the following theorem.

Theorem 6.1 (Engelfriet, Fülöp, Vogler [19], Theorem 4.12). A mapping is realized by a root-to-frontier tree transducer iff it is realized by a polynomial top-down tree series transducer over the semiring $\mathbb{B}$.

Let $Q_{i}^{\prime}=\left\{\left(q, z_{i}\right) \mid q \in Q\right\}, 1 \leq i \leq k$. Then there is a one-to-one correspondence between $Q_{1}^{\prime} \times \cdots \times Q_{k}^{\prime}$ and $Q^{k}$ by

$$
\left(\left(q_{1}, z_{1}\right), \ldots,\left(q_{k}, z_{k}\right)\right) \Leftrightarrow\left(q_{1}, \ldots, q_{k}\right),
$$

$q_{1}, \ldots, q_{k} \in Q$. A tree representation $\left(\mu_{k} \mid k \geq 0\right)$ is called nondeterministically simple iff

$$
\mu_{k}: \Sigma_{k} \rightarrow\left(A\left\langle\left\langle T_{\Sigma^{\prime}}\left(X^{\prime} \cup Y_{k}\right)\right\rangle\right\rangle\right)^{Q \times\left(Q_{1}^{\prime} \times \cdots \times Q_{k}^{\prime}\right)}, \quad k \geq 1 .
$$

If $\left(\mu_{k} \mid k \geq 0\right)$ is a nondeterministically simple tree representation then we will work with the isomorphic copies $\mu_{k}(\omega)^{\prime}$ of $\mu_{k}(\omega), k \geq 0$, in $\left(A\left\langle\left\langle T_{\Sigma^{\prime}}\left(X^{\prime} \cup\right.\right.\right.\right.$ $\left.\left.\left.\left.Y_{k}\right)\right\rangle\right\rangle\right)^{Q \times Q^{k}}, k \geq 0$. By Theorem 2.13,

$$
\mu_{k}(\omega)^{\prime}\left(P_{1}, \ldots, P_{k}\right)=\mu_{k}(\omega)\left[P_{1}, \ldots, P_{k}\right]
$$

for $P_{j} \in\left(A\left\langle\left\langle T_{\Sigma}\left(X \cup Y^{\prime}\right)\right\rangle\right\rangle\right)^{Q \times 1}, 1 \leq j \leq k$, and $\omega \in \Sigma_{k}, k \geq 0$. Hence, we can define a nondeterministic simple tree representation to be a family of mappings $\left(\mu_{k} \mid k \geq 0\right)$, where

$$
\begin{aligned}
& \mu_{k}: \Sigma_{k} \rightarrow\left(A\left\langle\left\langle T_{\Sigma^{\prime}}\left(X^{\prime} \cup Y_{k}\right)\right\rangle\right\rangle\right)^{Q \times Q^{k}}, \quad k \geq 1, \\
& \left.\mu_{0}: \Sigma_{0} \cup X \rightarrow\left(A\left\langle T_{\Sigma^{\prime}}\left(X^{\prime}\right)\right\rangle\right\rangle\right)^{Q \times 1} .
\end{aligned}
$$


The morphic extension of $\mu_{0}$ is again defined by $\mu\left(\omega\left(t_{1}, \ldots, t_{k}\right)\right)=\mu(\omega)\left(\mu\left(t_{1}\right), \ldots, \mu\left(t_{k}\right)\right)$, $\omega \in \Sigma_{k}, t_{1}, \ldots, t_{k} \in T_{\Sigma}(X), k \geq 1$, and, for $s \in A\left\langle\left\langle T_{\Sigma}(X)\right\rangle\right\rangle$, we define again $\mu(s)=\sum_{t \in T_{\Sigma}(X)}(s, t) \otimes \mu(t)$.

A nondeterministic simple tree series transducer is now a tree series transducer $\mathfrak{T}=(Q, \mu, S)$, where $\mu$ is a nondeterministic simple tree representation and $\|\mathfrak{T}\|(s)=S(\mu(s))=\sum_{q \in Q}\left(S_{q}, z_{1}\right) \mu(s)_{q}$ for $s \in A\left\langle\left\langle T_{\Sigma}(X)\right\rangle\right\rangle$.

We now introduce linear and nondeleting tree representations and tree series transducers. A tree $t \in T_{\Sigma}\left(X \cup Y_{k}\right), k \geq 1$, is called linear iff the variable $y_{j}$ appears at most once in $t, 1 \leq j \leq k$. A tree $t \in T_{\Sigma}\left(X \cup Y_{k}\right), k \geq 1$, is called nondeleting iff the variable $y_{j}$ appears at least once in $t, 1 \leq j \leq k$. A tree series $s \in A\left\langle\left\langle T_{\Sigma}\left(X \cup Y_{k}\right)\right\rangle\right\rangle, k \geq 1$, is called linear or nondeleting iff all $t \in \operatorname{supp}(s)$ are linear or nondeleting, respectively. A nondeterministic simple tree representation $\mu$ is called linear or nondeleting iff all entries of $\mu(\omega), \omega \in \Sigma_{k}$, $k \geq 1$, are linear or nondeleting tree series, respectively. A nondeterministic simple tree series transducer $\mathfrak{T}=(Q, \mu, S)$ is called linear or nondeleting iff $\mu$ is linear or nondeleting, respectively.

Theorem 6.2 (Kuich [39]) Let, for some $k \geq 1, s \in A\left\langle\left\langle T_{\Sigma}\left(X \cup Y_{k}\right)\right\rangle\right\rangle$ be linear and nondeleting, and $s_{i_{j}} \in A\left\langle\left\langle T_{\Sigma}(X)\right\rangle\right\rangle, a_{i_{j}} \in A$ for $i_{j} \in I_{j}, 1 \leq j \leq k$. Then

$$
s\left(\sum_{i_{1} \in I_{1}} a_{i_{1}} s_{i_{1}}, \ldots, \sum_{i_{k} \in I_{k}} a_{i_{k}} s_{i_{k}}\right)=\sum_{i_{1} \in I_{1}} \ldots \sum_{i_{k} \in I_{k}} a_{i_{1}} \ldots a_{i_{k}} s\left(s_{i_{1}}, \ldots, s_{i_{k}}\right) .
$$

Theorem 6.3 Let $\omega \in \Sigma_{k}, k \geq 1, s_{1}, \ldots, s_{k} \in A\left\langle\left\langle T_{\Sigma}(X)\right\rangle\right\rangle$, and $\mu$ be a linear and nondeleting tree representation with state set $Q$. Then

$$
\mu(\omega)\left(\mu\left(s_{1}\right), \ldots, \mu\left(s_{k}\right)\right)=\mu\left(\bar{\omega}\left(s_{1}, \ldots, s_{k}\right)\right) .
$$

Proof. We first compute the left side of the equality of the theorem for index $q \in Q:$

$$
\begin{aligned}
& \left.\sum_{q_{1}, \ldots, q_{k} \in Q}^{\mu(\omega)\left(\mu\left(s_{1}\right), \ldots, \mu\left(s_{k}\right)\right)_{q}=} \mu(\omega)_{q,\left(q_{1}, \ldots, q_{k}\right)}\right) \\
& \sum_{q_{1}, \ldots, q_{k} \in Q} \mu(\omega)_{q,\left(q_{1}, \ldots, q_{k}\right)}\left(\sum_{\left.\left.q_{1}\right)_{q_{1}}, \ldots, \mu\left(s_{k}\right)_{q_{k}}\right)=}\left(s_{1}, t_{1}\right) \mu\left(t_{1}\right)_{q_{1}}, \ldots, \sum_{t_{1} \in T_{\Sigma}(X)}\left(s_{k}, t_{k}\right) \mu\left(t_{k}\right)_{q_{k}}\right)= \\
& \sum_{q_{1}, \ldots, q_{k} \in Q} \sum_{t_{1}, \ldots, t_{k} \in T_{\Sigma}(X)}\left(s_{1}, t_{1}\right) \cdots\left(s_{k}, t_{k}\right) \mu(\omega)_{q,\left(q_{1}, \ldots, q_{k}\right)}\left(\mu\left(t_{1}\right)_{q_{1}}, \ldots, \mu\left(t_{k}\right)_{q_{k}}\right)= \\
& \sum_{t_{1}, \ldots, t_{k} \in T_{\Sigma}(X)}\left(s_{1}, t_{1}\right) \cdots\left(s_{k}, t_{k}\right) \mu\left(\omega\left(t_{1}, \ldots, t_{k}\right)\right)_{q} .
\end{aligned}
$$

Here the third equality follows by the assumption that $\mu$ is a linear and nondeleting tree representation and by Theorem 6.2. We now compute the right 
side of the equality of the theorem for index $q \in Q$ :

$$
\begin{aligned}
& \mu\left(\sum_{t \in T_{\Sigma}(X)}^{\mu\left(\bar{\omega}\left(s_{1}, \ldots, s_{k}\right)\right)_{q}=}\left(\sum_{\omega\left(t_{1}, \ldots, t_{k}\right)=t}^{\mu}\left(s_{1}, t_{1}\right) \cdots\left(s_{k}, t_{k}\right)\right) t\right)_{q}= \\
& \sum_{t \in T_{\Sigma}(X)}\left(\sum_{\omega\left(t_{1}, \ldots, t_{k}\right)=t}^{\left(s_{1}, t_{1}\right) \cdots\left(s_{k}, t_{k}\right) \mu\left(\omega\left(t_{1}, \ldots, t_{k}\right)\right)_{q} .} .\right.
\end{aligned}
$$

Since both sides of the equation of the theorem coincide, the theorem is proven.

If $A$ is an idempotent continuous semiring then $\sum_{i \in I} a=a$ for all $a \in A$, and we do not need the condition in Theorem 6.2 that $s$ is nondeleting.

Theorem 6.4 Let $A$ be an idempotent commutative continuous semiring. Let, for some $k \geq 1, s \in A\left\langle\left\langle T_{\Sigma}\left(X \cup Y_{k}\right)\right\rangle\right\rangle$ be linear, and $s_{i_{j}} \in A\left\langle\left\langle T_{\Sigma}(X)\right\rangle\right\rangle, a_{i_{j}} \in$ $\{0,1\} \subseteq A$ for $i_{j} \in I_{j}, 1 \leq j \leq k$. Then

$$
s\left(\sum_{i_{1} \in I_{1}} a_{i_{1}} s_{i_{1}}, \ldots, \sum_{i_{k} \in I_{k}} a_{i_{k}} s_{i_{k}}\right)=\sum_{i_{1} \in I_{1}} \ldots \sum_{i_{k} \in I_{k}} a_{i_{1}} \ldots a_{i_{k}} s\left(s_{i_{1}}, \ldots, s_{i_{k}}\right) .
$$

Proof. Assume that the variables $z_{j+1}, \ldots, z_{k}$ do not appear in $s$ but the variables $z_{1}, \ldots, z_{j}$ do. Then $s\left(z_{1}, \ldots, z_{k}\right)=s^{\prime}\left(z_{1}, \ldots, z_{j}\right)$ and

$$
\begin{aligned}
& s\left(\sum_{i_{1} \in I_{1}} a_{i_{1}} s_{i_{1}}, \ldots, \sum_{i_{k} \in I_{k}} a_{i_{k}} s_{i_{k}}\right)= \\
& s^{\prime}\left(\sum_{i_{1} \in I_{1}} a_{i_{1}} s_{i_{1}}, \ldots, \sum_{i_{j} \in I_{j}} a_{i_{j}} s_{i_{j}}\right)= \\
& \sum_{i_{1} \in I_{1}} \ldots \sum_{i_{j} \in I_{j}} a_{i_{1}} \ldots a_{i_{j}} s^{\prime}\left(s_{i_{1}}, \ldots, s_{i_{j}}\right)= \\
& \sum_{i_{1} \in I_{1}} \ldots \sum_{i_{j} \in I_{j}} \sum_{i_{j+1} \in I_{j+1}} \ldots \sum_{i_{k} \in I_{k}} a_{i_{1}} \ldots a_{i_{k}} s\left(s_{i_{1}}, \ldots, s_{i_{k}}\right) .
\end{aligned}
$$

Theorem 6.4 implies at once the following corollary to Theorem 6.3.

Corollary 6.5 Let $\omega \in \Sigma_{k}, k \geq 1, s_{1}, \ldots, s_{k} \in \mathbb{B}\left\langle\left\langle T_{\Sigma}(X)\right\rangle\right.$, and $\mu$ be a linear tree representation with state set $Q$. Then

$$
\mu(\omega)\left(\mu\left(s_{1}\right), \ldots, \mu\left(s_{k}\right)\right)=\mu\left(\bar{\omega}\left(s_{1}, \ldots, s_{k}\right)\right) .
$$

In case of the Boolean semiring $\mathbb{B}$ it is easy to see by Example 6.1 that our polynomial tree series transducers do not preserve the recognizability of tree series. (See also the example in the last paragraph of page 18 of Gecseg, Steinby [25].) On the other hand, linear root-to-frontier tree transducers 
do preserve recognizability of tree languages. (See Thatcher [56]; and Gécseg, Steinby [24], Theorem 2.7, Lemma 6.5 and Corollary 6.6.) In the rest of this section we show that linear nondeleting recognizable tree transducers do preserve recognizability of tree series. We show this by a construction based on finite recognizable systems. $\left.\left.\left.Z_{n}\right)\right\rangle\right\rangle$.

A system $z_{i}=p_{i}, 1 \leq i \leq n$ is called recognizable iff each $p_{i}$ is in $A^{\text {rec }}\left\langle\left\langle T_{\Sigma}(X \cup\right.\right.$

We show that the least solution of a finite recognizable system has recognizable components.

Theorem 6.6 Let $z_{i}=p_{i}, 1 \leq i \leq n$, be a finite recognizable system with least solution $\sigma$. Then $\sigma_{i} \in A^{\mathrm{rec}}\left\langle\left\langle T_{\Sigma}(X)\right\rangle\right\rangle$ for all $1 \leq i \leq n$.

Proof. Without loss of generality let $z_{i}=p_{i}, 1 \leq i \leq n$, be a proper finite recognizable system. Since $p_{i} \in A^{\text {rec }}\left\langle\left\langle T_{\Sigma}\left(X \cup Z_{n}\right)\right\rangle\right\rangle, 1 \leq i \leq n$, there exist proper finite polynomial systems $y_{i j}=q_{i j}, 1 \leq j \leq m_{i}, m_{i} \geq 1$, where the $y_{i j}$ are new variables and $q_{i j} \in A\left\langle T_{\Sigma}\left(X \cup Z_{n} \cup\left\{y_{i 1}, \ldots, y_{i m_{i}}\right\}\right)\right\rangle$, such that the $y_{i 1}$-components of their least solutions $\tau_{i}$ are equal to $p_{i}$. Consider now the proper finite polynomial system $z_{i}=q_{i 1}\left(z_{1}, \ldots, z_{n}, y_{i 1}, \ldots, y_{i m_{i}}\right), y_{i j}=$ $q_{i j}\left(z_{1}, \ldots, z_{n}, y_{i 1}, \ldots, y_{i m_{i}}\right), 1 \leq j \leq m_{i}, 1 \leq i \leq n$, and observe that it has a unique solution. We claim that this unique solution is given by $\sigma \cup$ $\left(\left(\tau_{i}\right)_{j}\left(\sigma_{1}, \ldots, \sigma_{n}\right) \mid 1 \leq j \leq m_{i}, 1 \leq i \leq n\right)$. Substitution of this vector yields, for $1 \leq j \leq m_{i}, 1 \leq i \leq n$,

$$
\begin{aligned}
& q_{i 1}\left(\sigma_{1}, \ldots, \sigma_{n},\left(\tau_{i}\right)_{1}\left(\sigma_{1}, \ldots, \sigma_{n}\right), \ldots,\left(\tau_{i}\right)_{m_{i}}\left(\sigma_{1}, \ldots, \sigma_{n}\right)\right)= \\
& \quad\left(\tau_{i}\right)_{1}\left(\sigma_{1}, \ldots, \sigma_{n}\right)=p_{i}\left(\sigma_{1}, \ldots, \sigma_{n}\right)=\sigma_{i}, \\
& q_{i j}\left(\sigma_{1}, \ldots, \sigma_{n},\left(\tau_{i}\right)_{1}\left(\sigma_{1}, \ldots, \sigma_{n}\right), \ldots,\left(\tau_{i}\right)_{m_{i}}\left(\sigma_{1}, \ldots, \sigma_{n}\right)\right)=\left(\tau_{i}\right)_{j}\left(\sigma_{1}, \ldots, \sigma_{n}\right) .
\end{aligned}
$$

Hence $\sigma \cup\left(\left(\tau_{i}\right)_{j}\left(\sigma_{1}, \ldots, \sigma_{n}\right) \mid 1 \leq j \leq m_{i}, 1 \leq i \leq n\right)$ is the unique solution of the proper finite polynomial system and $\sigma \in\left(A^{\text {rec }}\left\langle\left\langle T_{\Sigma}(X)\right\rangle\right\rangle\right)^{n \times 1}$.

Consider a finite system $y_{i}=p_{i}\left(y_{1}, \ldots, y_{n}\right), 1 \leq i \leq n$, where $p_{i} \in A\left\langle\left\langle T_{\Sigma}(X \cup\right.\right.$ $\left.\left.\left.Y_{n}\right)\right\rangle\right\rangle$, and a linear nondeleting tree representation $\mu=\left(\mu_{k} \mid k \geq 0\right)$ with state set $Q$, where $\mu_{k}: \Sigma_{k} \rightarrow\left(A\left\langle\left\langle T_{\Sigma^{\prime}}\left(X^{\prime} \cup Z_{k}\right)\right\rangle\right\rangle\right)^{Q \times Q^{k}}, k \geq 1$, and $\mu_{0}: \Sigma_{0} \cup X \rightarrow$ $\left(A\left\langle\left\langle T_{\Sigma^{\prime}}\left(X^{\prime}\right)\right\rangle\right\rangle\right)^{Q \times 1}$. Let $\left(y_{i}\right)_{q}, 1 \leq i \leq n, q \in Q$, be new variables and denote $Y_{Q}^{k}=\left\{\left(y_{i}\right)_{q} \mid 1 \leq i \leq k, q \in Q\right\}$. Extend the definition of $\mu$ to the domain $\Sigma \cup X \cup Y_{n}$, by

$$
\mu_{0}: Y_{n} \rightarrow\left(A\left\langle\left\langle T_{\Sigma^{\prime}}\left(Y_{Q}^{n}\right)\right\rangle\right\rangle\right)^{Q \times 1},
$$

where $\mu\left(y_{j}\right)_{q}=\left(y_{j}\right)_{q}, 1 \leq j \leq n, q \in Q$. By this extension, we obtain that

$$
\mu: T_{\Sigma}\left(X \cup Y_{n}\right) \rightarrow\left(A\left\langle\left\langle T_{\Sigma^{\prime}}\left(X^{\prime} \cup Y_{Q}^{n}\right)\right\rangle\right\rangle\right)^{Q \times 1} .
$$

Lemma 6.7 Consider $s\left(y_{1}, \ldots, y_{n}\right) \in A\left\langle\left\langle T_{\Sigma}\left(X \cup Y_{n}\right)\right\rangle\right\rangle$ and a linear nondeleting tree representation $\mu$ with domain $\Sigma \cup X \cup Y_{n}$. Let $s_{1}, \ldots, s_{n} \in A\left\langle\left\langle T_{\Sigma}(X)\right\rangle\right.$. Then

$$
\mu(s)\left[\mu\left(s_{j}\right)_{q} /\left(y_{j}\right)_{q}, 1 \leq j \leq n, q \in Q\right]=\mu\left(s\left(s_{1}, \ldots, s_{n}\right)\right) .
$$


Proof. We first consider a tree $t \in T_{\Sigma}\left(X \cup Y_{n}\right)$ and show by induction on the form of $t$ that $\mu(t)\left[\mu\left(s_{j}\right)_{q} /\left(y_{j}\right)_{q}, 1 \leq j \leq n, q \in Q\right]=\mu\left(t\left(s_{1}, \ldots, s_{n}\right)\right)$.

(i) For $t=y_{i}, 1 \leq i \leq n$, we obtain $\mu\left(y_{i}\right)\left[\mu\left(s_{j}\right) / \mu\left(y_{j}\right), 1 \leq j \leq n\right]=\mu\left(s_{i}\right)=$ $\mu\left(y_{i}\left(s_{1}, \ldots, s_{n}\right)\right)$.

(ii) For $t=x, x \in \Sigma_{0} \cup X$, we obtain $\mu(x)\left[\mu\left(s_{j}\right) / \mu\left(y_{j}\right), 1 \leq j \leq n\right]=\mu(x)=$ $\mu\left(x\left(s_{1}, \ldots, s_{n}\right)\right)$.

(iii) For $t=\omega\left(t_{1}, \ldots, t_{k}\right), \omega \in \Sigma_{k}, t_{1}, \ldots, t_{k} \in T_{\Sigma}\left(X \cup Y_{n}\right), k \geq 1$, we obtain

$$
\begin{aligned}
& \mu\left(\omega\left(t_{1}, \ldots, t_{k}\right)\right)\left[\mu\left(s_{j}\right) / \mu\left(y_{j}\right), 1 \leq j \leq n\right]= \\
& \mu(\omega)\left(\mu\left(t_{1}\right)\left[\mu\left(s_{j}\right) / \mu\left(y_{j}\right), 1 \leq j \leq n\right], \ldots, \mu\left(t_{k}\right)\left[\mu\left(s_{j}\right) / \mu\left(y_{j}\right), 1 \leq j \leq n\right]\right)= \\
& \mu(\omega)\left(\mu\left(t_{1}\left(s_{1}, \ldots, s_{n}\right)\right), \ldots, \mu\left(t_{k}\left(s_{1}, \ldots, s_{n}\right)\right)\right)= \\
& \mu\left(\bar{\omega}\left(t_{1}\left(s_{1}, \ldots, s_{n}\right), \ldots, t_{k}\left(s_{1}, \ldots, s_{n}\right)\right)\right)= \\
& \mu\left(\left(\bar{\omega}\left(t_{1}, \ldots, t_{k}\right)\right)\left(s_{1}, \ldots, s_{n}\right)\right) .
\end{aligned}
$$

Here we have applied the induction hypothesis in the second equality and Theorem 6.3 in the third equality.

Finally, we obtain

$$
\begin{aligned}
& \mu(s)\left[\mu\left(s_{j}\right) / \mu\left(y_{j}\right), 1 \leq j \leq n\right]= \\
& \sum_{t \in T_{\Sigma}\left(X \cup Y_{n}\right)}(s, t) \otimes \mu(t)\left[\mu\left(s_{j}\right) / \mu\left(y_{j}\right), 1 \leq j \leq n\right]= \\
& \sum_{t \in T_{\Sigma}\left(X \cup Y_{n}\right)}(s, t) \otimes \mu\left(t\left(s_{1}, \ldots, s_{n}\right)\right)= \\
& \mu\left(\sum_{t \in T_{\Sigma}\left(X \cup Y_{n}\right)}(s, t) t\left(s_{1}, \ldots, s_{n}\right)\right)=\mu\left(s\left(s_{1}, \ldots, s_{n}\right)\right) .
\end{aligned}
$$

Theorem 6.8 Consider a linear nondeleting tree representation $\mu$ with domain $\Sigma \cup X \cup Y_{n}$. Let $y_{i}=p_{i}\left(y_{1}, \ldots, y_{n}\right), 1 \leq i \leq n$, where $p_{i} \in A\left\langle\left\langle T_{\Sigma}\left(X \cup Y_{n}\right)\right\rangle\right.$, be a finite system with least solution $\sigma$. Then $\mu(\sigma)$ is the least solution of the finite system $\mu\left(y_{i}\right)=\mu\left(p_{i}\left(y_{1}, \ldots, y_{n}\right)\right), 1 \leq i \leq n$.

Proof. Let $\left(\sigma^{j} \mid j \in \mathbb{N}\right)$ and $\left(\tau^{j} \mid j \in \mathbb{N}\right)$ be the approximation sequences of $y_{i}=p_{i}\left(y_{1}, \ldots, y_{n}\right), 1 \leq i \leq n$, and $\mu\left(y_{i}\right)=\mu\left(p_{i}\left(y_{1}, \ldots, y_{n}\right)\right), 1 \leq i \leq n$, respectively. We claim that $\tau_{i}^{j}=\mu\left(\sigma_{i}^{j}\right), 1 \leq i \leq n, j \geq 0$, and show it by induction on $j$. The case $j=0$ is clear. Let $j \geq 0$. Then, for $1 \leq i \leq n$,

$$
\begin{aligned}
& \tau_{i}^{j+1}=\mu\left(p_{i}\left(y_{1}, \ldots, y_{n}\right)\right)\left[\tau_{k}^{j} / \mu\left(y_{k}\right), 1 \leq k \leq n\right]= \\
& \mu\left(p_{i}\left(y_{1}, \ldots, y_{n}\right)\right)\left[\mu\left(\sigma_{k}^{j}\right) / \mu\left(y_{k}\right), 1 \leq k \leq n\right]= \\
& \mu\left(p_{i}\left(\sigma_{1}^{j}, \ldots, \sigma_{n}^{j}\right)\right)=\mu\left(\sigma_{i}^{j+1}\right) .
\end{aligned}
$$

Here we have applied the induction hypothesis in the second equality and Lemma 6.7 in the third equality. The claim now implies our theorem.

A nondeterministically simple tree representation $\mu=\left(\mu_{k} \mid k \geq 0\right)$ is called recognizable iff $\mu_{k}(\omega) \in\left(A^{\mathrm{rec}}\left\langle\left\langle T_{\Sigma^{\prime}}\left(X^{\prime} \cup Z_{k}\right)\right\rangle\right\rangle\right)^{Q \times Q^{k}}$ for $\omega \in \Sigma_{k}, k \geq 1$, and $\mu_{0}(\omega) \in\left(A^{\mathrm{rec}}\left\langle\left\langle T_{\Sigma^{\prime}}\left(X^{\prime}\right)\right\rangle\right\rangle\right)^{Q \times 1}$ for $\omega \in \Sigma_{0} \cup X$. A nondeterministically simple tree series transducer $\mathfrak{T}=(Q, \mu, S)$ is called recognizable iff $\mu$ is a recognizable tree representation. 
Theorem 6.9 Consider a linear nondeleting recognizable tree representation $\mu$. Let $s$ be in $A^{\text {rec }}\left\langle\left\langle T_{\Sigma}(X)\right\rangle\right\rangle$. Then $\mu(s)$ is in $\left(A^{\text {rec }}\left\langle\left\langle T_{\Sigma^{\prime}}\left(X^{\prime}\right)\right\rangle\right\rangle\right)^{Q \times 1}$.

Proof. By Corollary 3.6, $s$ is a component of a finite simple polynomial system $y_{i}=p_{i}, 1 \leq i \leq n$. By Theorem $6.8, \mu(s)$ is a component of the finite recognizable system $\mu\left(y_{i}\right)=\mu\left(p_{i}\right), 1 \leq i \leq n$. Hence, Theorem 6.6 proves our theorem.

Corollary 6.10 Consider a linear nondeleting recognizable tree series transducer $\mathfrak{T}$ and a recognizable tree series $s$. Then $\|\mathfrak{T}\|(s)$ is again recognizable.

The proof of Corollary 6.10 is mainly based on the application of Theorem 6.3. If $\mathbb{B}$ is the basic semiring and we apply Corollary 6.5 instead of Theorem 6.3 then we get the following corollaries.

Corollary 6.11 Let $\mathbb{B}$ be the basic semiring. Consider a linear recognizable tree series transducer $\mathfrak{T}$ and a recognizable tree series $s$. Then $\|\mathfrak{T}\|(s)$ is again recognizable.

Corollary 6.12 (Thatcher [56], and Gécseg, Steinby [24], Corollary 6.6.) Linear root-to-frontier tree transducers preserve recognizability.

\section{$7 \quad$ Full abstract families of tree series}

Full abstract families of tree series (briefly, full AFTs) are families of tree series closed under linear nondeleting recognizable tree transductions and certain specific operations. We will show that the families of recognizable tree series and of algebraic tree series are full AFTs. Our first construction will show that the mappings realized by linear nondeleting recognizable tree series transducers are closed under functional composition. The construction is analogous to the construction of Engelfriet [18] in Lemma 4.2 (see also Gécseg, Steinby [24], Theorem IV.3.15).

Recall that $Z_{Q}=\left\{\left(z_{i}\right)_{q} \mid i \geq 1, q \in Q\right\}$ and $Z_{Q}^{k}=\left\{\left(z_{i}\right)_{q} \mid 1 \leq i \leq k, q \in Q\right\}$ for $k \geq 1$. We now define, for $r_{1}, \ldots, r_{k} \in Q$, the operator

$$
\varphi_{r_{1}, \ldots, r_{k}}: A\left\langle\left\langle T_{\Sigma}\left(X \cup Z_{Q}^{k}\right)\right\rangle\right\rangle \rightarrow A\left\langle\left\langle T_{\Sigma}\left(X \cup\left\{\left(z_{1}\right)_{r_{1}}, \ldots,\left(z_{k}\right)_{r_{k}}\right\}\right)\right\rangle\right\rangle
$$

as follows: For $s \in A\left\langle\left\langle T_{\Sigma}\left(X \cup Z_{Q}^{k}\right)\right\rangle\right\rangle$ and $t \in T_{\Sigma}\left(X \cup\left\{\left(z_{1}\right)_{r_{1}}, \ldots,\left(z_{k}\right)_{r_{k}}\right\}\right)$,

$$
\left(\varphi_{r_{1}, \ldots, r_{k}}(s), t\right)= \begin{cases}(s, t) & \text { iff the variables }\left(z_{1}\right)_{r_{1}}, \ldots,\left(z_{k}\right)_{r_{k}} \\ 0 & \text { appear exactly once in } t, \\ \text { otherwise. }\end{cases}
$$

Let $\mu^{\prime}$ be a linear nondeleting recognizable tree representation with state set $Q_{1}$ mapping $\Sigma \cup X$ into matrices with entries in $A^{\text {rec }}\left\langle\left\langle T_{\Sigma^{\prime}}\left(X^{\prime} \cup Z\right)\right\rangle\right.$. Furthermore, 
let $\mu^{\prime \prime}$ be an extended linear nondeleting recognizable tree representation with state set $Q_{2}$ mapping $\Sigma^{\prime} \cup X^{\prime} \cup Z$ into matrices with entries in $A^{\text {rec }}\left\langle\left\langle T_{\Sigma^{\prime \prime}}\left(X^{\prime \prime} \cup\right.\right.\right.$ $\left.\left.\left.Z \cup Z_{Q_{2}}\right)\right\rangle\right\rangle$. Define the recognizable tree representation $\mu$ with state set $Q_{1} \times Q_{2}$ mapping $\Sigma \cup X$ into matrices with entries in $A^{\text {rec }}\left\langle\left\langle T_{\Sigma^{\prime \prime}}\left(X^{\prime \prime} \cup Z\right)\right\rangle\right\rangle$ by

$$
\begin{aligned}
& \mu_{0}(x)_{\left(q_{1}, q_{2}\right)}=\mu_{0}^{\prime \prime}\left(\mu_{0}^{\prime}(x)_{q_{1}}\right)_{q_{2}}, \quad \text { for } x \in \Sigma_{0} \cup X, q_{1} \in Q_{1}, q_{2} \in Q_{2}, \\
& \mu_{k}(\omega)_{\left(q_{1}, q_{2}\right),\left(\left(r_{1}, s_{1}\right), \ldots,\left(r_{k}, s_{k}\right)\right)}= \\
& \quad \varphi_{s_{1}, \ldots, s_{k}}\left(\mu_{k}^{\prime \prime}\left(\mu_{k}^{\prime}(\omega)_{q_{1}},\left(r_{1}, \ldots, r_{k}\right)\right)_{q_{2}}\right)\left[z_{1} /\left(z_{1}\right)_{s_{1}}, \ldots, z_{k} /\left(z_{k}\right)_{s_{k}}\right] \\
& \quad \text { for } \omega \in \Sigma_{k}, k \geq 1, q_{1}, r_{1}, \ldots, r_{k} \in Q_{1}, q_{2}, s_{1}, \ldots, s_{k} \in Q_{2} .
\end{aligned}
$$

Then, by Kuich [40], Lemma 2.3, $\left(\mu_{k} \mid k \geq 0\right)$ is a linear nondeleting recognizable tree representation and, for $t \in T_{\Sigma}(X)$ and $q_{1} \in Q_{1}, q_{2} \in Q_{2}$,

$$
\mu(t)_{\left(q_{1}, q_{2}\right)}=\mu^{\prime \prime}\left(\mu^{\prime}(t)_{q_{1}}\right)_{q_{2}} .
$$

This construction yields the first theorem of this section.

Theorem 7.1 Let $\mu^{\prime}$ (resp. $\mu^{\prime \prime}$ ) be a linear nondeleting recognizable tree representation with state set $Q_{1}$ (resp. $\left.Q_{2}\right)$ mapping $\Sigma \cup X\left(\right.$ resp. $\left.\Sigma^{\prime} \cup X^{\prime}\right)$ into matrices with entries in $A^{\text {rec }}\left\langle\left\langle T_{\Sigma^{\prime}}\left(X^{\prime} \cup Z\right)\right\rangle\right\rangle$ (resp. $\left.A^{\text {rec }}\left\langle\left\langle T_{\Sigma^{\prime \prime}}\left(X^{\prime \prime} \cup Z\right)\right\rangle\right\rangle\right)$. Let $\mathfrak{T}_{1}=\left(Q_{1}, \mu^{\prime}, S_{1}\right)$ and $\mathfrak{T}_{2}=\left(Q_{2}, \mu^{\prime \prime}, S_{2}\right)$ be linear nondeleting recognizable tree transducers. Then there exists a linear nondeleting recognizable tree transducer $\mathfrak{T}$ such that $\|\mathfrak{T}\|(s)=\left\|\mathfrak{T}_{2}\right\|\left(\left\|\mathfrak{T}_{1}\right\|(s)\right)$ for all $s \in A\left\langle\left\langle T_{\Sigma}(X)\right\rangle\right\rangle$.

Proof. The linear nondeleting recognizable tree transducer $\mathfrak{T}=\left(Q_{1} \times Q_{2}, \mu\right.$, $\left.S_{1} \odot S_{2}\right)$ is defined by the linear nondeleting recognizable tree representation $\mu$ constructed above.

Let $\left(S_{1}\right)_{q_{1}}=a_{q_{1}} z_{1}, a_{q_{1}} \in A, q_{1} \in Q_{1}$, and $\left(S_{2}\right)_{q_{2}}=b_{q_{2}} z_{1}, b_{q_{2}} \in A, q_{2} \in Q_{2}$. Then $\left(S_{1} \odot S_{2}\right)_{\left(q_{1}, q_{2}\right)}=a_{q_{1}} b_{q_{2}} z_{1}$ for $q_{1} \in Q_{1}, q_{2} \in Q_{2}$. We now obtain, for $s \in A\left\langle\left\langle T_{\Sigma}(X)\right\rangle\right\rangle$,

$$
\begin{aligned}
& \left\|\mathfrak{T}_{2}\right\|\left(\left\|\mathfrak{T}_{1}\right\|(s)\right)=\sum_{q_{2} \in Q_{2}} b_{q_{2}} \sum_{t_{2} \in T_{\Sigma^{\prime}}\left(X^{\prime}\right)}\left(\left\|\mathfrak{T}_{1}\right\|(s), t_{2}\right) \mu_{2}\left(t_{2}\right)_{q_{2}}= \\
& \sum_{q_{2} \in Q_{2}} b_{q_{2}} \sum_{t_{2} \in T_{\Sigma^{\prime}}\left(X^{\prime}\right)}\left(\sum_{q_{1} \in Q_{1}} a_{q_{1}} \sum_{t_{1} \in T_{\Sigma}(X)}\left(s, t_{1}\right) \mu_{1}\left(t_{1}\right)_{q_{1}}, t_{2}\right) \mu_{2}\left(t_{2}\right)_{q_{2}}= \\
& \sum_{q_{1} \in Q_{1}} \sum_{q_{2} \in Q_{2}} a_{q_{1}} b_{q_{2}} \sum_{t_{1} \in T_{\Sigma}(X)}\left(s, t_{1}\right) \sum_{t_{2} \in T_{\Sigma^{\prime}}\left(X^{\prime}\right)}\left(\mu_{1}\left(t_{1}\right)_{q_{1}}, t_{2}\right) \mu_{2}\left(t_{2}\right)_{q_{2}}= \\
& \sum_{\left.q_{1}, q_{2}\right) \in Q_{1} \times Q_{2}} a_{q_{1}} b_{q_{2}} \sum_{t_{1} \in T_{\Sigma}(X)}\left(s, t_{1}\right) \mu\left(t_{1}\right)_{\left(q_{1}, q_{2}\right)}= \\
& \|\mathfrak{T}\|(s) .
\end{aligned}
$$

We make the following convention for the rest of Section 7: The set $\Sigma_{\infty}$ (resp. $X_{\infty}$ ) is a fixed infinite ranked alphabet (resp. infinite alphabet) and $\Sigma$ (resp. $X$ ), possibly provided with indices, is a finite subalphabet of $\Sigma_{\infty}$ (resp. $\left.X_{\infty}\right)$. Our basic semiring will be $A\left\langle\left\langle T_{\Sigma_{\infty}}\left(X_{\infty}\right)\right\rangle\right\rangle$.

Any nonempty subset of $\bigcup_{\Sigma \subset \Sigma_{\infty}} \bigcup_{X \subset X_{\infty}} A\left\langle\left\langle T_{\Sigma}(X)\right\rangle\right\rangle$ is called family of tree series. A mapping

$$
\tau: \bigcup_{\Sigma \subset \Sigma_{\infty}} \bigcup_{X \subset X_{\infty}} A\left\langle\left\langle T_{\Sigma}(X)\right\rangle\right\rangle \rightarrow \bigcup_{\Sigma \subset \Sigma_{\infty}} \bigcup_{X \subset X_{\infty}} A\left\langle\left\langle T_{\Sigma}(X)\right\rangle\right\rangle
$$


is called linear nondeleting recognizable tree series transduction iff there exist $\Sigma, X, \Sigma^{\prime}, X^{\prime}$ such that, for $s \in A\left\langle\left\langle T_{\Sigma}(X)\right\rangle\right\rangle, \tau(s) \in A\left\langle\left\langle T_{\Sigma^{\prime}}\left(X^{\prime}\right)\right\rangle\right\rangle$, and, for $s \notin$ $A\left\langle\left\langle T_{\Sigma}(X)\right\rangle\right\rangle, \tau(s)=0$ and there exists a linear nondeleting recognizable tree series transducer $\mathfrak{T}$ such that $\tau(s)=\|\mathfrak{T}\|(s)$ for $s \in A\left\langle\left\langle T_{\Sigma}(X)\right\rangle\right\rangle$.

For a family $\mathfrak{L}$ of tree series, we define

$$
\begin{aligned}
& \mathfrak{M}(\mathfrak{L})=\{\tau(s) \mid s \in \mathfrak{L} \text { and } \tau \text { is a linear nondeleting } \\
& \text { recognizable tree series transduction\}. }
\end{aligned}
$$

Observe that, by Theorem $7.1, \mathfrak{M}(\mathfrak{M}(\mathfrak{L}))=\mathfrak{M}(\mathfrak{L})$. A family $\mathfrak{L}$ of tree series is said to be closed under linear nondeleting recognizable tree series transductions, and is called a recognizable tree series cone iff $\mathfrak{L}=\mathfrak{M}(\mathfrak{L})$.

We first consider recognizable tree series. Theorem 6.9 yields at once the next theorem.

Theorem $7.2 A^{\mathrm{rec}}\left\langle\left\langle T_{\Sigma_{\infty}}\left(X_{\infty}\right)\right\rangle\right\rangle$ is a recognizable tree series cone.

Theorem 7.3 Let $\mathfrak{L}$ be a recognizable tree series cone and assume that $\mathfrak{L}$ contains some tree series s such that $(s, x)=1$ for some $x \in X_{\infty}$. Then $A^{\text {rec }}\left\langle\left\langle T_{\Sigma_{\infty}}\left(X_{\infty}\right)\right\rangle \subseteq \subseteq\right.$ $\mathfrak{L}$.

Proof. Consider a recognizable tree series $r$ and the linear nondeleting recognizable tree transducer $\mathfrak{T}=\left(\{q\},\left(\mu_{k} \mid k \geq 0\right), z_{1}\right)$, where $\mu_{0}(x)=r, \mu_{0}\left(x^{\prime}\right)=0$ for $x^{\prime} \neq x, x^{\prime} \in X_{\infty}$, and $\mu_{k}(\omega)=0, \omega \in \Sigma_{\infty}$, of $\operatorname{rank} k \geq 0$. Then $\|\mathfrak{T}\|(s)=r$.

Analogous to the REC-closed families of tree series of Bozapalidis, Rahonis [12] we introduce equationally closed families of tree series. A family $\mathfrak{L}$ of tree series is called equationally closed whenever the following conditions are satisfied:

(i) $0 \in \mathfrak{L}$

(ii) If $s_{1}, s_{2} \in \mathfrak{L}$ then $s_{1}+s_{2} \in \mathfrak{L}$.

(iii) If $\omega \in \Sigma_{\infty}$ is of rank $k \geq 0$ and $s_{1}, \ldots, s_{k} \in \mathfrak{L}$ then $\bar{\omega}\left(s_{1}, \ldots, s_{k}\right) \in \mathfrak{L}$; if $x \in X_{\infty}$ then $x \in \mathfrak{L}$.

(iv) If $s \in \mathfrak{L}$ and $x \in X_{\infty}$ then the least solution $\mu x . s$ of the equation $x=s$ is in $\mathfrak{L}$.

Hence, a family $\mathfrak{L}$ of tree series is equationally closed iff $\langle\mathfrak{L},+, 0,(\bar{\omega} \mid \omega \in$ $\left.\left.\Sigma_{\infty}\right) \cup X_{\infty}\right\rangle$ is a distributive $\Sigma_{\infty} \cup X_{\infty}$-algebra that satisfies condition (iv), i. e., our "rational" operations are 0 , addition, top-catenation and least solutions of equations. (Observe that we do not ask for the closure under substitution as Bozapalidis, Rahonis [12] do for their REC-closed families of tree languages.)

Theorem 7.4 $A^{\mathrm{rec}}\left\langle\left\langle T_{\Sigma_{\infty}}\left(X_{\infty}\right)\right\rangle\right\rangle$ is an equationally closed family of tree series. 
Proof. By Theorem 4.1.

We are now ready to introduce full AFTs. We use the notation $\mathfrak{F}(\mathfrak{L}), \mathfrak{L}$ a family of tree series, for the smallest equationally closed family of tree series that is closed under linear nondeleting recognizable tree series transductions and contains $\mathfrak{L}$. A family $\mathfrak{L}$ of tree series is called full AFT iff $\mathfrak{L}=\mathfrak{F}(\mathfrak{L})$.

Theorem $7.5 A^{\mathrm{rec}}\left\langle\left\langle T_{\Sigma_{\infty}}\left(X_{\infty}\right)\right\rangle\right\rangle$ is a full AFT.

Proof. By Theorems 7.2 and 7.4.

We now consider algebraic tree series.

Theorem 7.6 $A^{\mathrm{alg}}\left\langle\left\langle T_{\Sigma_{\infty}}\left(X_{\infty}\right)\right\rangle\right\rangle$ is an equationally closed family of tree series.

Proof. By Theorem 5.13.

We will show that $A^{\operatorname{alg}}\left\langle\left\langle T_{\Sigma_{\infty}}\left(X_{\infty}\right)\right\rangle\right\rangle$ is a full AFT closed under linear nondeleting algebraic tree series transductions. Some definitions and results are needed before that result.

A nondeterministically simple tree representation $\mu=\left(\mu_{k} \mid k \geq 0\right)$ is called algebraic iff $\mu_{k}(\omega) \in\left(A^{\text {alg }}\left\langle\left\langle T_{\Sigma^{\prime}}\left(X^{\prime} \cup Z_{k}\right)\right\rangle\right\rangle\right)^{Q \times Q^{k}}$ for $\omega \in \Sigma_{k}, k \geq 1$, and $\mu_{0}(\omega) \in\left(A^{\text {alg }}\left\langle\left\langle T_{\Sigma^{\prime}}\left(X^{\prime}\right)\right\rangle\right\rangle\right)^{Q \times 1}$ for $\omega \in \Sigma_{0} \cup X$. A nondeterministically simple tree series transducer $\mathfrak{T}=(Q, \mu, S)$ is called algebraic iff $\mu$ is an algebraic tree representation. Linear nondeleting algebraic tree series transductions are defined analogously to linear nondeleting recognizable tree series transductions.

Theorem 7.7 (Kuich [41], Corollary 3.6.) Let $\mathfrak{T}$ be a linear nondeleting algebraic tree series transducer and $s$ be an algebraic tree series. Then $\|\mathfrak{T}\|(s)$ is again algebraic.

Theorem 7.8 Let $\mathfrak{T}_{1}$ and $\mathfrak{T}_{2}$ be linear nondeleting algebraic tree transducers. Then there exists a linear nondeleting algebraic tree transducer $\mathfrak{T}$ such that $\|\mathfrak{T}\|(s)=\left\|\mathfrak{T}_{2}\right\|\left(\left\|\mathfrak{T}_{1}\right\|(s)\right)$ for all $s \in A\left\langle\left\langle T_{\Sigma}(X)\right\rangle\right\rangle$.

Proof. The construction of $\mathfrak{T}$ from $\mathfrak{T}_{1}$ and $\mathfrak{T}_{2}$ is analogous to the construction in the proof of Theorem 7.1. Theorem 7.7 proves that $\mu$ is algebraic.

For a family $\mathfrak{L}$ of tree series, we define

$$
\begin{aligned}
\mathfrak{M}^{\text {alg }}(\mathfrak{L})=\{\tau(s) \mid & s \in \mathfrak{L} \text { and } \tau \text { is a linear nondeleting } \\
& \text { algebraic tree series transduction }\} .
\end{aligned}
$$

Observe that by Theorem $7.8, \mathfrak{M}^{\text {alg }}\left(\mathfrak{M}^{\text {alg }}(\mathfrak{L})\right)=\mathfrak{M}^{\text {alg }}(\mathfrak{L})$. A family $\mathfrak{L}$ of tree series is said to be closed under linear nondeleting algebraic tree series transductions, and is called an algebraic tree series cone iff $\mathfrak{L}=\mathfrak{M}^{\text {alg }}(\mathfrak{L})$.

Theorem 7.9 Let $\mathfrak{L}$ be an algebraic tree series cone and assume that $\mathfrak{L}$ contains some tree series $s$ such that $(s, x)=1$ for some $x \in X_{\infty}$. Then $A^{\text {alg }}\left\langle\left\langle T_{\Sigma_{\infty}}\left(X_{\infty}\right)\right\rangle\right\rangle \subseteq$ $\mathfrak{L}$. 
Proof. Similar to the proof of Theorem 7.3.

Theorem 7.10 $A^{\text {alg }}\left\langle\left\langle T_{\Sigma_{\infty}}\left(X_{\infty}\right)\right\rangle\right\rangle$ is an algebraic cone.

Proof. By Theorems 7.6 and 7.7 .

Corollary 7.11 $A^{\mathrm{alg}}\left\langle\left\langle T_{\Sigma_{\infty}}\left(X_{\infty}\right)\right\rangle\right\rangle$ is a full AFT that is closed under linear nondeleting algebraic tree series transductions.

\section{Connections to formal power series}

The application of the yield-mapping to formal tree series yields formal power series. We will first show that macro power series are the yield of algebraic tree series. Here, macro power series are introduced as a generalization of the OI languages of Fischer [22] and the indexed languages of Aho [1]. Moreover, we show a Kleene Theorem for macro power series and indexed languages. Then we show that algebraic power series are the yield of recognizable tree series. Finally, we prove that the yield of a full abstract family of tree series is a full abstract family of power series.

We now introduce macro power series. Let $\Phi=\left\{G_{1}, \ldots, G_{n}\right\}, \Phi \cap \Sigma=\emptyset$, where $G_{i}$ has rank $r_{i}, 1 \leq i \leq n$, be a finite ranked alphabet of function variables. We define $T(\Phi, X)$ to be the set of words over $\Phi \cup X \cup\{(\} \cup\{)\} \cup\{$, satisfying the following conditions:

(i) $X \cup\{\varepsilon\} \subset T(\Phi, X)$;

(ii) if $t_{1}, t_{2} \in T(\Phi, X)$ then $t_{1} t_{2} \in T(\Phi, X)$;

(iii) if $G \in \Phi$, where $G$ is of rank $r \geq 0$, and $t_{1}, \ldots, t_{r} \in T(\Phi, X)$ then $G\left(t_{1}, \ldots, t_{r}\right) \in T(\Phi, X)$.

The words of $T(\Phi, X)$ are called terms over $\Phi$ and $X$. By $A\langle\langle T(\Phi, X)\rangle\rangle$ (resp. $A\langle T(\Phi, X)\rangle)$ we denote the set of power series whose supports are subsets (resp. finite subsets) of $T(\Phi, X)$.

Let $D^{\prime}=A\left\langle\left\langle\left(X \cup Z_{r_{1}}\right)^{*}\right\rangle\right\rangle \times \ldots \times A\left\langle\left\langle\left(X \cup Z_{r_{n}}\right)^{*}\right\rangle\right\rangle$ and consider power series $s_{i} \in A\left\langle\left\langle T\left(\Phi, X \cup Z_{r_{i}}\right)\right\rangle\right\rangle, 1 \leq i \leq n$. Then each $s_{i}$ induces a function $\bar{s}_{i}: D^{\prime} \rightarrow$ $A\left\langle\left\langle\left(X \cup Z_{r_{i}}\right)^{*}\right\rangle\right\rangle$. For $\left(\tau_{1}, \ldots, \tau_{n}\right) \in D^{\prime}$, we define inductively $\bar{s}_{i}\left(\tau_{1}, \ldots, \tau_{n}\right)$ to be

(i) $z_{m}$ if $s_{i}=z_{m}, 1 \leq m \leq r_{i} ; x$ if $s_{i}=x, x \in X$;

(ii) $\bar{t}_{1}\left(\tau_{1}, \ldots, \tau_{n}\right) \bar{t}_{2}\left(\tau_{1}, \ldots, \tau_{n}\right)$ if $s_{i}=t_{1} t_{2}, t_{1}, t_{2} \in T\left(\Phi, X \cup Z_{r_{i}}\right)$;

(iii) $\tau_{j}\left(\bar{t}_{1}\left(\tau_{1}, \ldots, \tau_{n}\right), \ldots, \bar{t}_{r_{j}}\left(\tau_{1}, \ldots, \tau_{n}\right)\right)$ if $s_{i}=G_{j}\left(t_{1}, \ldots, t_{r_{j}}\right), G_{j} \in \Phi$, $t_{1}, \ldots, t_{r_{j}} \in T\left(\Phi, X \cup Z_{r_{i}}\right)$

(iv) $a \cdot \bar{t}\left(\tau_{1}, \ldots, \tau_{n}\right)$ if $s_{i}=a t, a \in A, t \in T\left(\Phi, X \cup Z_{r_{i}}\right)$; 
(v) $\sum_{j \in J} \bar{r}_{j}\left(\tau_{1}, \ldots, \tau_{n}\right)$ if $s_{i}=\sum_{j \in J} r_{j}, r_{j} \in A\left\langle\left\langle T\left(\Phi, X \cup Z_{r_{i}}\right)\right\rangle\right\rangle, j \in J$, where $J$ is an arbitrary index set.

The mappings $\bar{s}_{i}, 1 \leq i \leq n$, are continuous and the mapping $\bar{s}: D^{\prime} \rightarrow D^{\prime}$, where $\bar{s}=\left\langle\bar{s}_{1}, \ldots, \bar{s}_{n}\right\rangle$, is again continuous. This is proved similarly to the proof of the continuity of the mappings defined in connection with algebraic tree systems (below Theorem 5.1).

A macro system $\mathfrak{S}=(\Phi, Z, X, E)$ (with function variables in $\Phi$, variables in $Z$ and terminal symbols in $X$ ) has a set $E$ of formal equations

$$
G_{i}\left(z_{1}, \ldots, z_{r_{i}}\right)=s_{i}\left(z_{1}, \ldots, z_{r_{i}}\right), 1 \leq i \leq n,
$$

where each $s_{i}$ is in $A\left\langle T\left(\Phi, X \cup Z_{r_{i}}\right)\right\rangle$.

A solution to the macro system $\mathfrak{S}$ is given by $\left(\tau_{1}, \ldots, \tau_{n}\right) \in D^{\prime}$ such that $\tau_{i}=$ $\bar{s}_{i}\left(\tau_{1}, \ldots, \tau_{n}\right), 1 \leq i \leq n$, i. e., by any fixed point $\left(\tau_{1}, \ldots, \tau_{n}\right)$ of $\bar{s}=\left\langle\bar{s}_{1}, \ldots, \bar{s}_{n}\right\rangle$. A solution $\left(\sigma_{1}, \ldots, \sigma_{n}\right)$ of the macro system $\mathfrak{S}$ is called least solution iff $\sigma_{i} \leq \tau_{i}$, $1 \leq i \leq n$, for all solutions $\left(\tau_{1}, \ldots, \tau_{n}\right)$ of $\mathfrak{S}$. Since the least solution of $\mathfrak{S}$ is nothing else than the least fixpoint of $\bar{s}=\left\langle\bar{s}_{1}, \ldots, \bar{s}_{n}\right\rangle$, the least solution of the macro system $\mathfrak{S}$ exists in $D^{\prime}$.

Theorem 8.1 (Kuich [43], Theorem 5.1.) Let $\mathfrak{S}=\left(\Phi, Z, \Sigma,\left\{G_{i}=s_{i} \mid 1 \leq i \leq\right.\right.$ $n\})$ be a macro system, where $s_{i} \in A\left\langle T\left(\Phi, X \cup Z_{r_{i}}\right)\right\rangle$. Then the least solution of this macro system $\mathfrak{S}$ exists in $D^{\prime}$ and equals

$$
\operatorname{fix}(\bar{s})=\sup \left(\bar{s}^{i}(0) \mid i \in \mathbb{N}\right),
$$

where $\bar{s}^{i}$, is the $i$-th iterate of the mapping $\bar{s}=\left\langle\bar{s}_{1}, \ldots, \bar{s}_{n}\right\rangle: D^{\prime} \rightarrow D^{\prime}$.

Theorem 8.1 indicates how we can compute an approximation to the least solution of a macro system. The approximation sequence $\left(\tau^{j} \mid j \in \mathbb{N}\right)$, where each $\tau^{j} \in D^{\prime}$, associated with the macro system $\mathfrak{S}=\left(\Phi, Z, \Sigma,\left\{G_{i}=s_{i} \mid 1 \leq\right.\right.$ $i \leq n\}$ ) is defined as follows:

$$
\tau^{0}=0, \quad \tau^{j+1}=\bar{s}\left(\tau^{j}\right), j \in \mathbb{N} .
$$

Clearly, the least solution $\operatorname{fix}(\bar{s})$ of $\mathfrak{S}$ is equal to $\sup \left(\tau^{j} \mid j \in \mathbb{N}\right)$. A macro system $\mathfrak{S}=\left(\Phi \cup\left\{G_{0}\right\}, Z, \Sigma,\left\{G_{i}=s_{i} \mid 0 \leq i \leq n\right\}, G_{0}\right)$ (with function variables in $\Phi \cup\left\{G_{0}\right\}$, variables in $Z$, terminal symbols in $\Sigma$ ) with initial function variable $G_{0}$ is a macro system $\left(\Phi \cup\left\{G_{0}\right\}, Z, \Sigma,\left\{G_{i}=s_{i} \mid 0 \leq i \leq n\right\}\right)$ such that $G_{0}$ has rank 0 . Let $\left(\tau_{0}, \tau_{1}, \ldots, \tau_{n}\right)$ be the least solution of $\left(\Phi \cup\left\{G_{0}\right\}, Z, \Sigma,\left\{G_{i}=s_{i} \mid\right.\right.$ $0 \leq i \leq n\})$. Then $\tau_{0}$ is called the initial component of the least solution. Observe that $\tau_{0} \in A\left\langle\left\langle T_{\Sigma}(X)\right\rangle\right\rangle$ contains no variables of $Z$.

A power series $r$ in $A\left\langle\left\langle X^{*}\right\rangle\right\rangle$ is called macro power series iff $r$ is the initial component of the least solution of a macro system with initial function variable.

Analogous to the proof of Theorem 3.4 of Engelfriet, Schmidt [20] it can be shown that, in the case of the Boolean semiring, $r \in \mathbb{B}\left\langle\left\langle X^{*}\right\rangle\right\rangle$ is a macro 
power series iff $\operatorname{supp}(r) \in X^{*}$ is an OI language in the sense of Definition 3.10 of Fischer [22]. Moreover, by Theorem 5.3 of Fischer [22], $r \in \mathbb{B}\left\langle\left\langle X^{*}\right\rangle\right\rangle$ is a macro power series iff $\operatorname{supp}(r) \in X^{*}$ is an indexed language (see Aho [1]).

We now define a mapping yd : $A\left\langle\left\langle T_{\Sigma \cup \Phi}(X \cup Z)\right\rangle\right\rangle \rightarrow A\langle\langle T(\Phi, X \cup Z)\rangle$. For $s \in A\left\langle\left\langle T_{\Sigma \cup \Phi}(X \cup Z)\right\rangle\right\rangle, \operatorname{yd}(s)$ is called the yield of $s ; \operatorname{yd}(s)$ is defined inductively to be

(i) $z_{m}$ if $s=z_{m} \in Z$; $x$ if $s=x, x \in X$;

(ii) $\operatorname{yd}\left(t_{1}\right) \ldots \operatorname{yd}\left(t_{r}\right)$ if $s=f\left(t_{1}, \ldots, t_{r}\right), f \in \Sigma_{r}, t_{1}, \ldots, t_{r} \in T_{\Sigma \cup \Phi}(X \cup Z)$, $r \geq 0 ;\left(\right.$ observe that $\operatorname{yd}(f)=\varepsilon$ if $\left.f \in \Sigma_{0}\right)$;

(iii) $G_{i}\left(\operatorname{yd}\left(t_{1}\right), \ldots, \operatorname{yd}\left(t_{r_{i}}\right)\right)$ if $s=G_{i}\left(t_{1}, \ldots, t_{r_{i}}\right), t_{1}, \ldots, t_{r_{i}} \in T_{\Sigma \cup \Phi}(X \cup Z)$, $1 \leq i \leq n$

(iv) $\sum_{t \in T_{\Sigma \cup \Phi}(X \cup Z)}(s, t) \operatorname{yd}(t)$ if $s=\sum_{t \in T_{\Sigma \cup \Phi}(X \cup Z)}(s, t) t$.

Observe that $\operatorname{yd}(s) \in A\left\langle\left\langle(X \cup Z)^{*}\right\rangle\right\rangle$ if $s \in A\left\langle\left\langle T_{\Sigma}(X \cup Z)\right\rangle\right\rangle$. Hence, our mapping yd is an extension of the usual yield-mapping (see Gécseg, Steinby [25], Section 14).

We will connect algebraic tree series and macro power series by the yieldmapping in our next theorem.

Given an algebraic tree system $\mathfrak{S}=\left(\Phi, Z, X,\left\{G_{i}\left(z_{1}, \ldots, z_{r_{i}}\right)=s_{i} \mid 1 \leq i \leq\right.\right.$ $n\})$, we define the macro system $\operatorname{yd}(\mathfrak{S})$ to be $\operatorname{yd}(\mathfrak{S})=\left(\Phi, Z, X,\left\{G_{i}\left(z_{1}, \ldots, z_{r_{i}}\right)=\right.\right.$ $\left.\left.\operatorname{yd}\left(s_{i}\right) \mid 1 \leq i \leq n\right\}\right)$.

Theorem 8.2 (Kuich [43], Theorem 5.5.) If $\left(\tau_{1}, \ldots, \tau_{n}\right)$ is the least solution of the algebraic tree system $\mathfrak{S}$ then $\left(\operatorname{yd}\left(\tau_{1}\right), \ldots, \operatorname{yd}\left(\tau_{n}\right)\right)$ is the least solution of the macro system $\mathrm{yd}(\mathfrak{S})$.

Corollary 8.3 If $s$ is an algebraic tree series then $\mathrm{yd}(s)$ is a macro power series.

Corollary 8.4 Let $\{\bullet, e\} \subseteq \Sigma$, where $\bullet$ and e have rank 2 and 0 , respectively. Then a power series $r \in A\left\langle\left\langle X^{*}\right\rangle\right\rangle$ is a macro power series iff there exists an algebraic tree series $s \in A\left\langle\left\langle T_{\Sigma}(X)\right\rangle\right\rangle$ such that $\operatorname{yd}(s)=r$.

Example 8.1. Let $\mathfrak{S}=\left(\Phi, Z, \Sigma, E, Z_{0}\right)$ be the algebraic tree system specified by

(i) $\Phi=\left\{G_{0}, G_{1}, G_{2}, Z_{0}\right\}$, where the ranks of $G_{0}, G_{1}, G_{2}$ are 3 and the rank of $Z_{0}$ is 0 ;

(ii) $Z=\left\{z_{0}, z_{1}, z_{2}\right\}$;

(iii) $\Sigma=\Sigma_{2}=\{b\}, X=\left\{c_{1}, c_{2}\right\}$; 
(iv) the formal equations of $E$ are

$$
\begin{aligned}
& G_{0}\left(z_{0}, z_{1}, z_{2}\right)=G_{0}\left(G_{0}\left(z_{0}, z_{1}, z_{2}\right), G_{1}\left(z_{0}, z_{1}, z_{2}\right), G_{2}\left(z_{0}, z_{1}, z_{2}\right)\right)+b\left(z_{1}, z_{2}\right) \\
& G_{i}\left(z_{0}, z_{1}, z_{2}\right)=b\left(z_{i}, z_{i}\right), i=1,2 \\
& Z_{0}=G_{0}\left(0, c_{1}, c_{2}\right)
\end{aligned}
$$

Then the initial component of the least solution of $\mathfrak{S}$ is $\sum_{j \geq 0} b\left(t_{1}^{j}, t_{2}^{j}\right)$, where $t_{1}^{j}$ and $t_{2}^{j}, j \geq 0$, are defined in Example 5.1. The macro system $\operatorname{yd}(\mathfrak{S})=$ $\left(\Phi, Z, X, E^{\prime}, Z_{0}\right)$ is specified by the following formal equations of $E^{\prime}$ :

$$
\begin{aligned}
& G_{0}\left(z_{0}, z_{1}, z_{2}\right)=G_{0}\left(G_{0}\left(z_{0}, z_{1}, z_{2}\right), G_{1}\left(z_{0}, z_{1}, z_{2}\right), G_{2}\left(z_{0}, z_{1}, z_{2}\right)\right)+z_{1} z_{2} \\
& G_{i}\left(z_{0}, z_{1}, z_{2}\right)=z_{i} z_{i}, i=1,2 \\
& Z_{0}=G_{0}\left(0, c_{1}, c_{2}\right)
\end{aligned}
$$

The initial component of the least solution of $\operatorname{yd}(\mathfrak{S})$ is $\sum_{j \geq 0} c_{1}^{2^{j}} c_{2}^{2^{j}}$.

We now introduce macro power series expressions. Assume that $A, X, Z, \Phi_{\infty}$ and $U=\{+, \cdot, \mu,[]$,$\} are mutually disjoint. A word E$ over $A \cup X \cup Z \cup \Phi_{\infty} \cup U$ is a macro power series expression over $\left(A, X, Z, \Phi_{\infty}\right)$ iff

(i) $E$ is in $X \cup Z \cup\{\varepsilon\}$, or

(ii) $E$ is of one of the forms $\left[E_{1}+E_{2}\right],\left[E_{1} E_{2}\right], G\left(E_{1}, \ldots, E_{k}\right), a E_{1}$ or $\mu G . E_{1}$, where $E_{1}, \ldots, E_{k}$ are macro power series expressions over $\left(A, X, Z, \Phi_{\infty}\right)$ for $G \in \Phi_{\infty}$ of rank $k, k \geq 0$, and $a \in A$.

Each macro power series expression $E$ over $\left(A, X, Z, \Phi_{\infty}\right)$ denotes a formal power series $|E|$ in $A\langle\langle T(\Phi, X \cup Z)\rangle\rangle$, where $\Phi$ is some suitable finite subset of $\Phi_{\infty}$, according to the following conventions:

(i) If $E$ is in $X \cup Z \cup\{\varepsilon\}$ then $E$ denotes the term $E$, i. e., $|E|=E$.

(ii) For macro power series expressions $E_{1}, \ldots, E_{k}$ over $\left(A, X, Z, \Phi_{\infty}\right), G \in$ $\Phi_{\infty}$ of rank $k, k \geq 0, a \in A$, we define $\left|\left[E_{1}+E_{2}\right]\right|=\left|E_{1}\right|+\left|E_{2}\right|$, $\left|\left[E_{1} E_{2}\right]\right|=\left|E_{1}\right|\left|E_{2}\right|$, $\left|G\left(E_{1}, \ldots, E_{k}\right)\right|=\sum_{t_{1}, \ldots, t_{k} \in T(\Phi, X \cup Z)}\left(\left|E_{1}\right|, t_{1}\right) \ldots\left(\left|E_{k}\right|, t_{k}\right) G\left(t_{1}, \ldots, t_{k}\right)$, $\left|a E_{1}\right|=a\left|E_{1}\right|$ $\left|\mu G \cdot E_{1}\right|=\mu G \cdot\left|E_{1}\right|$.

We now define a "yield-mapping" $Y$, which maps algebraic tree series expressions over $\left(A, \Sigma, X, Z, \Phi_{\infty}\right)$ to macro power series expressions over $\left(A, X, Z, \Phi_{\infty}\right)$, in the following manner:

(i) if $E$ is in $X \cup Z$ then $\mathrm{Y}(E)=E$, 
(ii) for algebraic tree series expressions $E_{1}, \ldots, E_{k}$ over $\left(A, \Sigma, X, Z, \Phi_{\infty}\right), \omega \in$ $\Sigma$ of rank $k, G \in \Phi_{\infty}$ of rank $k, k \geq 0, a \in A$ we define

$\mathrm{Y}\left(\left[E_{1}+E_{2}\right]\right)=\left[\mathrm{Y}\left(E_{1}\right)+\mathrm{Y}\left(E_{2}\right)\right]$,

$\mathrm{Y}\left(\omega\left(E_{1}, \ldots, E_{k}\right)\right)=\left[\ldots\left[\mathrm{Y}\left(E_{1}\right) \mathrm{Y}\left(\mathrm{E}_{2}\right)\right] \cdots \mathrm{Y}\left(E_{k}\right)\right]$

(including $\mathrm{Y}(\omega)=\varepsilon$ for $k=0, \mathrm{Y}\left(\omega\left(E_{1}\right)\right)=\mathrm{Y}\left(E_{1}\right)$ for $k=1$ ),

$\mathrm{Y}\left(G\left(E_{1}, \ldots, E_{k}\right)\right)=G\left(\mathrm{Y}\left(E_{1}\right), \ldots, \mathrm{Y}\left(E_{k}\right)\right)$,

$\mathrm{Y}\left(a E_{1}\right)=a \mathrm{Y}\left(E_{1}\right)$

$\mathrm{Y}\left(\mu G . E_{1}\right)=\mu G . \mathrm{Y}\left(E_{1}\right)$.

We claim that $\operatorname{yd}(|E|)=|\mathrm{Y}(E)|$ for an algebraic tree series expression over $\left(A, \Sigma, X, Z, \Phi_{\infty}\right)$. The proof is by induction of the form of $E$. We only show the case $E=\mu G$. $E_{1}$. We obtain

$$
\operatorname{yd}(|E|)=\operatorname{yd}\left(\mu G \cdot\left|E_{1}\right|\right)=\mu G \cdot \operatorname{yd}\left(\left|E_{1}\right|\right)=\mu G \cdot\left|\mathrm{Y}\left(E_{1}\right)\right|=\left|\mathrm{Y}\left(\mu G \cdot E_{1}\right)\right|=|\mathrm{Y}(E)| .
$$

Here the second equality follows by the continuity of the mapping yd and the third equality follows by the induction hypothesis.

We now define the mappings $\Phi_{1}, \Phi_{2}, \Phi_{3}$ analogous to these mappings in Section 5 .

These considerations, together with Corollaries 5.18 and 8.4 imply the following result. It can be considered as a Kleene Theorem for macro power series.

Theorem 8.5 Let $\{\bullet, e\} \subseteq \Sigma$, where $\bullet$ and e have rank 2 and 0 , respectively. Then a power series $r \in A\left\langle\left\langle X^{*}\right\rangle\right\rangle$ is a macro power series iff there exists a macro power series expression $E$ over $\left(A, X, Z, \Phi_{\infty}\right)$ such that $r=|E|$, where $\Phi_{1}(E)=\Phi_{3}(E)=\emptyset$.

If the basic semiring is $\mathbb{B}$, then Theorem 8.5 can be considered as a Kleene Theorem for indexed languages.

Example 8.2. Consider the macro system $\mathfrak{M}=\left(\Phi, Z, X, E, G_{0}\right)$ with initial function variable $G_{0}$, specified by $\Phi=\Phi_{0} \cup \Phi_{2}, \Phi_{0}=\left\{G_{0}\right\}, \Phi_{2}=\{G\}, X=$ $\left\{c_{1}, c_{2}\right\}$ and $E=\left\{G_{0}=G\left(c_{1}, c_{2}\right), G\left(z_{1}, z_{2}\right)=G\left(z_{1}^{2}, z_{2}^{2}\right)+z_{1} z_{2}\right\}$. Since $\mathfrak{M}=$ $\operatorname{yd}(\mathfrak{S})$, where $\mathfrak{S}$ is defined in Example 5.2, we obtain that the initial component of the least solution of $\mathfrak{M}$ is given by $\sum_{j \geq 0} c_{1}^{j^{j}} c_{2}^{2^{j}}=\mid \mu G \cdot\left[G\left(\left[c_{1} c_{1}\right],\left[c_{2} c_{2}\right]\right)+\right.$ $\left.\left[c_{1} c_{2}\right]\right] \mid$. Observe that this macro power series expression is $\mathrm{Y}(E)$ where $E$ is the algebraic tree series expression given in Example 5.2.

We now show that algebraic power series are the yield of recognizable tree series.

Let $z_{i}=p_{i}, p_{i} \in A\left\langle\left\langle T_{\Sigma}\left(X \cup Z_{n}\right)\right\rangle\right\rangle, 1 \leq i \leq n$, be a simple proper finite polynomial system with least solution $\left(\sigma_{1}, \ldots, \sigma_{n}\right)$. Consider the proper algebraic system $z_{i}=\operatorname{yd}\left(p_{i}\right), \operatorname{yd}\left(p_{i}\right) \in A\left\langle\left\langle\left(X \cup Z_{n}\right)^{*}\right\rangle\right\rangle, 1 \leq i \leq n$. Then it is easily proved that its least solution is given by $\left(\operatorname{yd}\left(\sigma_{1}\right), \ldots, \operatorname{yd}\left(\sigma_{n}\right)\right)$. This proves the next theorem.

Theorem 8.6 If $s$ is a recognizable tree series then $\mathrm{yd}(s)$ is an algebraic power series. 
Corollary 8.7 Let $\{\bullet, e\} \subseteq \Sigma$, where $\bullet$ and e have rank 2 and 0 , respectively. Then a power series $r \in A\left\langle\left\langle X^{*}\right\rangle\right\rangle$ is algebraic iff there exists a tree series in $A^{\mathrm{rec}}\left\langle\left\langle T_{\Sigma}(X)\right\rangle\right\rangle$ such that $\mathrm{yd}(s)=r$.

For $A=\mathbb{N}^{\infty}$, Theorem 8.6 and Theorem 3.9 of Kuich [36] imply the following wellknown result of formal language theory. (See also Bucher, Maurer [13], Section 3.3, Gécseg, Steinby [25], Section 14, and Seidl [55].)

Theorem 8.8 Let $G$ be a context-free grammar. Then for $w \in L(G)$ there are $d(w)$ different leftmost derivations for $w$ in $G$ iff there are $d(w)$ nonisomorphic derivation trees of $G$ with result $w$.

The Kleene Theorems of Section 4 imply by Corollary 8.7 Kleene Theorems for algebraic power series and context-free languages. (See Kuich [37], Gruska [30].)

We now turn to the theory of full abstract families of tree series and make the following convention for the rest of Section 8: The set $\Sigma_{\infty}\left(\right.$ resp. $\left.X_{\infty}\right)$ is a fixed infinite ranked alphabet (resp. infinite alphabet) and $\Sigma$ (resp. $X$ ), possibly provided with indices, is a finite subalphabet of $\Sigma_{\infty}$ (resp. $\left.X_{\infty}\right)$. Moreover, $\Sigma_{\infty}$ contains a symbol $\bullet$ of rank 2 and a symbol $e$ of rank 0 .

We will show that, for a full $\operatorname{AFT} \mathfrak{L}$, yield $(\mathfrak{L})$ is a full abstract family of power series (briefly, $\operatorname{AFP})$. Here yield $(\mathfrak{L})=\{\operatorname{yd}(s) \mid s \in \mathfrak{L}\}$.

Theorem 8.9 Let $\mathfrak{L}$ be a equationally closed family of tree series. Then yield( $\mathfrak{L})$ is closed under addition, multiplication and star and contains 0 and 1.

Proof. (i) Let $r_{1}, r_{2} \in \operatorname{yield}(\mathfrak{L})$. Then there exist $s_{1}, s_{2} \in \mathfrak{L}$ auch that $\operatorname{yd}\left(s_{i}\right)=r_{i}$, $i=1,2$. Sincer $\mathfrak{L}$ is closed under addition, $s=s_{1}+s_{2} \in \mathfrak{L}$ and $\operatorname{yd}(s)=$ $r_{1}+r_{2} \in \operatorname{yield}(\mathfrak{L})$. Since $\mathfrak{L}$ is closed under top-catenation, $s^{\prime}=\bullet\left(s_{1}, s_{2}\right) \in \mathfrak{L}$ and $\operatorname{yd}\left(s^{\prime}\right)=r_{1} r_{2} \in \operatorname{yield}(\mathfrak{L})$.

(ii) Let $s \in \mathfrak{L}$ and assume that $x \in X_{\infty}$ does not appear in $s$. Consider the equation $x=\bullet(s, x)+e$. Its least solution $\mu x .(\bullet(s, x)+e)$ is in $\mathfrak{L}$. Hence, the least solution $\mu x \cdot y d(\bullet(s, x)+e)=\mu x \cdot(\operatorname{yd}(s) x+\varepsilon)=\operatorname{yd}(s)^{*}$ of $x=\operatorname{yd}(\bullet(s, x)+e)=$ $\operatorname{yd}(s) x+\varepsilon$ is in yield $(\mathfrak{L})$. Moreover, $\operatorname{yd}(0)=0$ and $0^{*}=1$ are in yield $(\mathfrak{L})$.

A multiplicative morphism

$$
\nu: X^{*} \rightarrow\left(A\left\langle\left\langle X^{\prime *}\right\rangle\right\rangle\right)^{Q \times Q}
$$

is called a power series representation. A power series representation $\nu$ is called rational (resp. algebraic, macro) iff the entries of $\nu(x), x \in X$, are rational (resp. algebraic, macro) power series. A power series transducer $\mathfrak{Z}=(Q, \nu, S, P)$ is called rational (resp. algebraic, macro) iff $\nu$ is a rational (resp. an algebraic, a macro) power series representation and the entries of $S$ and $P$ are rational (resp. algebraic, macro) power series. A power series transduction is called rational (resp. algebraic, macro) iff it is realized by a rational (resp. an algebraic, a macro) power series transducer. 
Lemma 8.10 Let $\nu$ be an algebraic power series representation defined by $\nu$ : $X \rightarrow\left(A^{\operatorname{alg}}\left\langle\left\langle X^{\prime *}\right\rangle\right\rangle\right)^{Q \times Q}$. Then there exists a linear nondeleting recognizable tree representation $\mu$ with state set $Q \times Q$ mapping $\Sigma \cup X$ into matrices with entries in $A^{\mathrm{rec}}\left\langle\left\langle T_{\Sigma^{\prime}}\left(X^{\prime} \cup Z\right)\right\rangle\right\rangle, \Sigma^{\prime}=\{\bullet, e\}$, such that, for all $s \in A\left\langle\left\langle T_{\Sigma}(X)\right\rangle\right\rangle$ and $q_{1}, q_{2} \in Q$

$$
\operatorname{yd}\left(\mu(s)_{\left(q_{1}, q_{2}\right)}\right)=\nu(\operatorname{yd}(s))_{q_{1}, q_{2}} .
$$

Proof. We construct $\mu=\left(\mu_{k} \mid k \geq 0\right)$ :

(i) For $x \in X$ and $q_{1}, q_{2} \in Q$ we construct $\mu_{0}(x)_{\left(q_{1}, q_{2}\right)}$ according to Corollary 8.7 with the property that $\operatorname{yd}\left(\mu_{0}(x)_{\left(q_{1}, q_{2}\right)}\right)=\nu(x)_{q_{1}, q_{2}}$.

(ii) For $\omega \in \Sigma_{0}$ and $q_{1}, q_{2} \in Q$ we define $\mu_{0}(\omega)_{\left(q_{1}, q_{2}\right)}=\delta_{q_{1}, q_{2}} e$, where $\delta$ is the Kronecker symbol; hence, $\operatorname{yd}\left(\mu(\omega)_{\left(q_{1}, q_{2}\right)}\right)=\delta_{q_{1}, q_{2}} \varepsilon=\nu(\varepsilon)_{q_{1}, q_{2}}$.

(iii) For $\omega \in \Sigma_{k}, k \geq 1$, and $q_{1}, q_{2}, r_{1}, \ldots, r_{k}, s_{1}, \ldots, s_{k} \in Q$, we define

$$
\begin{aligned}
\mu_{k}(\omega)_{\left(q_{1}, q_{2}\right),\left(\left(r_{1}, s_{1}\right), \ldots,\left(r_{k}, s_{k}\right)\right)}= & \delta_{q_{1}, r_{1}} \delta_{s_{1}, r_{2}} \ldots \delta_{s_{k-1}, r_{k}} \delta_{s_{k}, q_{2}} \\
& \bullet\left(z_{1}, \bullet\left(z_{2}, \bullet\left(\ldots \bullet\left(z_{k-1}, z_{k}\right) \ldots\right)\right) .\right.
\end{aligned}
$$

We first consider a tree $t \in T_{\Sigma}(X)$ and show that $\operatorname{yd}\left(\mu(t)_{\left(q_{1}, q_{2}\right)}\right)=\nu(\operatorname{yd}(t))_{q_{1}, q_{2}}$, $q_{1}, q_{2} \in Q$. The proof is by induction on the structure of trees in $T_{\Sigma}(X)$. The induction basis is true by (i) and (ii). Let now $t=\omega\left(t_{1}, \ldots, t_{k}\right), \omega \in \Sigma_{k}, k \geq 1$, $t_{1}, \ldots, t_{k} \in T_{\Sigma}(X)$. Then we obtain, for $q_{1}, q_{2} \in Q$,

$$
\begin{aligned}
& \operatorname{yd}\left(\mu(t)_{\left(q_{1}, q_{2}\right)}\right)=\operatorname{yd}\left(\mu\left(\omega\left(t_{1}, \ldots, t_{k}\right)\right)_{\left(q_{1}, q_{2}\right)}\right)= \\
& \operatorname{yd}\left(\sum_{r_{1}, \ldots, r_{k} \in Q} \sum_{s_{1}, \ldots, s_{k} \in Q} \mu(\omega)_{\left(q_{1}, q_{2}\right),\left(\left(r_{1}, s_{1}\right), \ldots,\left(r_{k}, s_{k}\right)\right)}\right. \\
& \operatorname{yd}\left(\sum_{r_{1}, \ldots, r_{k} \in Q} \sum_{s_{1}, \ldots, s_{k} \in Q} \delta_{q_{1}, r_{1}} \delta_{s_{1}, r_{2}} \ldots \delta_{s_{k-1}, r_{k}} \delta_{s_{k}, q_{2}}\right. \\
& \left.\bullet\left(\mu\left(t_{1}\right)_{\left(r_{1}, s_{1}\right)} \bullet\left(\mu\left(t_{2}\right)_{\left(r_{2}, s_{2}\right)}, \ldots\left(\ldots, \delta_{s^{\prime}}, \ldots\left(\mu\left(t_{k-1}\right)_{\left(r_{k-1}, s_{k-1}\right)}, \mu\left(t_{k}\right)_{\left(r_{k}, s_{k}\right)}\right) \ldots\right)\right)\right)\right)= \\
& \sum_{s_{1}, \ldots, s_{k-1} \in Q} \operatorname{yd}\left(\mu\left(t_{1}\right)_{\left(q_{1}, s_{1}\right)}\right) \operatorname{yd}\left(\mu\left(t_{2}\right)_{\left(s_{1}, s_{2}\right)}\right) \ldots \\
& \ldots \operatorname{yd}\left(\mu\left(t_{k-1}\right)_{\left(s_{k-2}, s_{k-1}\right)}\right) \operatorname{yd}\left(\mu\left(t_{k}\right)_{\left(s_{k-1}, q_{2}\right)}\right)= \\
& \sum_{s_{1}, \ldots, s_{k-1} \in Q} \nu\left(\operatorname{yd}\left(t_{1}\right)\right)_{q_{1}, s_{1}} \nu\left(\operatorname{yd}\left(t_{2}\right)\right)_{s_{1}, s_{2}} \ldots \\
& \ldots \nu\left(\operatorname{yd}\left(t_{k-1}\right)\right)_{s_{k-2}, s_{k-1}} \nu\left(\operatorname{yd}\left(t_{k}\right)\right)_{s_{k-1}, q_{2}}= \\
& \nu\left(\operatorname{yd}\left(t_{1}\right) \ldots \operatorname{yd}\left(t_{k}\right)\right)_{q_{1}, q_{2}}=\nu(\operatorname{yd}(t))_{q_{1}, q_{2}} .
\end{aligned}
$$

Hence, for $s \in A\left\langle\left\langle T_{\Sigma}(X)\right\rangle\right\rangle$ and $q_{1}, q_{2} \in Q$,

$$
\begin{aligned}
& \operatorname{yd}\left(\mu(s)_{\left(q_{1}, q_{2}\right)}\right)=\sum_{t \in T_{\Sigma}(X)}(s, t) \operatorname{yd}\left(\mu(t)_{\left(q_{1}, q_{2}\right)}\right)= \\
& \sum_{t \in T_{\Sigma}(X)}(s, t) \nu(\operatorname{yd}(t))_{q_{1}, q_{2}}=\nu(\operatorname{yd}(s))_{q_{1}, q_{2}} .
\end{aligned}
$$

A nonempty family of power series is called algebraic cone iff it is closed under algebraic power series transductions. Observe that each algebraic cone is a (rational) cone, i. e., a family of power series closed under rational power series transductions.

Theorem 8.11 Let $\mathfrak{L}$ be a full AFT. Then yield $(\mathfrak{L})$ is an algebraic cone. 
Proof. Let $s \in \mathfrak{L}, s \in A\left\langle\left\langle T_{\Sigma}(X)\right\rangle, r=\mathrm{yd}(s)\right.$, and $\mathfrak{Z}=(Q, \nu, S, P)$ be an algebraic transducer. We will show that $\|\mathfrak{Z}\|(r) \in A\left\langle\left\langle X^{\prime *}\right\rangle\right\rangle$ is again in yield( $(\mathfrak{L})$. Observe that $\|\mathfrak{Z}\|(r)=S \nu(r) P=\sum_{q_{1}, q_{2} \in Q} S_{q_{1}} \nu(r)_{q_{1}, q_{2}} P_{q_{2}}$, where $S_{q}, P_{q} \in$ $A^{\text {alg }}\left\langle\left\langle X^{\prime *}\right\rangle\right\rangle, q \in Q$. By Corollary 8.7 there exist $s_{q}, p_{q} \in A^{\text {rec }}\left\langle\left\langle T_{\Sigma^{\prime}}\left(X^{\prime}\right)\right\rangle\right\rangle, \bullet, e \in$ $\Sigma^{\prime}$, such that $\operatorname{yd}\left(s_{q}\right)=S_{q}, \operatorname{yd}\left(p_{q}\right)=P_{q}, q \in Q$. By Lemma 8.10 there exists a linear nondeleting recognizable tree representation $\mu$ with state set $Q \times Q$ such that $\operatorname{yd}\left(\mu(s)_{\left(q_{1}, q_{2}\right)}\right)=\nu(r)_{q_{1}, q_{2}}$ for all $q_{1}, q_{2}$. Since $\mathfrak{L}$ is equationally closed, $\sum_{q_{1}, q_{2} \in Q} \bullet\left(s_{q_{1}}, \bullet\left(\mu(s)_{\left(q_{1}, q_{2}\right)}, p_{q_{2}}\right)\right)$ is in $\mathfrak{L}$. Hence,

$$
\begin{aligned}
& \operatorname{yd}\left(\sum_{q_{1}, q_{2} \in Q} \bullet\left(s_{q_{1}}, \bullet\left(\mu(s)_{\left(q_{1}, q_{2}\right)}, p_{q_{2}}\right)\right)\right)= \\
& \sum_{q_{1}, q_{2} \in Q} \operatorname{yd}\left(s_{q_{1}}\right) \operatorname{yd}\left(\mu(s)_{\left(q_{1}, q_{2}\right)}\right) \operatorname{yd}\left(p_{q_{2}}\right)= \\
& \sum_{q_{1}, q_{2} \in Q} S_{q_{1}} \nu(r)_{q_{1}, q_{2}} P_{q_{2}}=\|\mathfrak{Z}\|(r)
\end{aligned}
$$

is in $\operatorname{yield}(\mathfrak{L})$.

Corollary 8.12 Let $\mathfrak{L}$ be a full AFT. Then yield $(\mathfrak{L})$ is a full AFP that is closed under algebraic transductions.

Corollary 8.13 The family of algebraic power series is a full AFP closed under algebraic transductions.

Corollary 8.14 The family of algebraic power series is a full AFP closed under substitutions.

Theorem 8.15 Let $\mathfrak{L}$ be a full AFT closed under algebraic tree series transductions. Then yield $(\mathfrak{L})$ is a full AFP closed under macro power series transductions.

Proof. Similar to the proof of Theorem 8.11.

Corollary 8.16 The family of macro power series is a full AFP closed under macro power series transductions.

Corollary 8.17 The family of macro power series is a full AFP closed under substitution.

We now turn to the language case, i. e., our basic semiring is now $\mathfrak{P}\left(T_{\Sigma_{\infty}}\left(X_{\infty}\right)\right)$. We use without mentioning the isomorphism between $\mathfrak{P}\left(T_{\Sigma_{\infty}}\left(X_{\infty}\right)\right)$ and $\mathbb{B}\left\langle\left\langle T_{\Sigma_{\infty}}\left(X_{\infty}\right)\right\rangle\right\rangle$.

A family $\mathfrak{L}$ of tree languages is called equationally closed iff $\langle\mathfrak{L}, \cup, \emptyset,(\bar{\omega} \mid$ $\left.\left.\omega \in \Sigma_{\infty}\right) \cup X_{\infty}\right\rangle$ is a distributive $\Sigma_{\infty} \cup X_{\infty}$-algebra that satisfies the following condition:

If $L \in \mathfrak{L}$ and $x \in X_{\infty}$ then the least solution $\mu x . L$ of the tree language equation $x=L$ is in $\mathfrak{L}$. 
Define $\hat{\mathfrak{F}}(\mathfrak{L})$ to be the smallest equationally closed family of tree languages that is closed under linear nondeleting recognizable tree transductions and contains $\mathfrak{L}$. A family $\mathfrak{L}$ of tree languages is called full abstract family of tree languages iff $\mathfrak{L}=\hat{\mathfrak{F}}(\mathfrak{L})$.

We now connect our full abstract families of tree languages with full AFLs (see Salomaa [54], Ginsburg [26] and Berstel [4]).

Theorem 8.18 Let $\mathfrak{L}$ be a full abstract family of tree languages. Then yield( $\mathfrak{L})$ is a full AFL that is closed under algebraic transductions.

A substitution $\sigma$ is called context-free iff $\sigma(x)$ is a context-free language for each $x \in X$.

Corollary 8.19 Let $\mathfrak{L}$ be a full abstract family of tree languages. Then yield( $\mathfrak{L})$ is a full AFL that is closed under context-free substitutions.

Corollary 8.20 The family of context-free languages is an AFL closed under substitution.

Corollary 8.21 (Aho [1], Theorem 3.4.) The family of indexed languages is an AFL closed under substitution. 


\section{References}

[1] Aho, A. V.: Indexed grammars - an extension of context-free grammars. JACM 15(1968) 647-671.

[2] Adamek J., Nelson, E., Reiterman J.: Tree constructions of free continuous algebras. J. Comput. System Sci. 24(1982) 114-146.

[3] Bekić, H.: Definable operations in general algebras, and the theory of automata and flowcharts. Tech. Report, IBM Labor, Wien, 1967.

[4] Berstel, J.: Transductions and Context-Free Languages. Teubner, 1979.

[5] Berstel, J., Reutenauer, C.: Recognizable formal power series on trees. Theor. Comput. Sci. 18(1982) 115-148.

[6] Bloom, St. L., Ésik, Z.: Iteration Theories. EATCS Monographs on Theoretical Computer Science. Springer, 1993.

[7] Bozapalidis, S.: Effective construction of the syntactic algebra of a recognizable series on trees. Acta Inf. 28(1991) 351-363.

[8] Bozapalidis, S.: Alphabetic tree relations. Theoret. Comput. Sci. 99(1992) 177-211.

[9] Bozapalidis, S.: Convex algebras, convex modules and formal power series on trees. Aristotle University of Thessaloniki, 1995.

[10] Bozapalidis, S.: Equational elements in additive algebras. Theory Comput. Systems 32(1999) 1-33.

[11] Bozapalidis, S.: Context-free series on trees. Information and Computation 169(2001) 186-229.

[12] Bozapalidis, S., Rahonis, G.: On two families of forests. Acta Inf. 31(1994) $235-260$

[13] Bucher, W., Maurer, H.: Theoretische Grundlagen der Programmiersprachen. B. I. Wissenschaftsverlag, 1984.

[14] Comon, H., Dauchet, M., Gilleron, R., Jaquemard, F., Lugiez, D., Tison, S., Tommasi, M.: Tree Automata-Techniques and Applications, Manuscript, 193 pages.

[15] Courcelle, B.: Equivalences and transformations of regular systemsApplications to recursive program schemes and grammars. Theor. Comp. Sci. 42(1986) 1-122.

[16] De Bakker, J. W., Scott, D.: A theory of programs, IBM Seminar, Vienna, 1969. 
[17] Eilenberg, S.: Automata, Languages and Machines. Vol. A. Academic Press, 1974.

[18] Engelfriet, J.: Bottom-up and top-down tree transformations - a comparison. Math. Systems Theory 9(1975) 198-231.

[19] Engelfriet, J., Fülöp, Z., Vogler, H.: Bottom-up and Top-down Tree Series Transducers. To appear in J. Automata, Languages and Combinatorics.

[20] Engelfriet, J., Schmidt, E. M.: IO and OI. I. J. Comput. Systems Sci. 15(1977) 328-353.

[21] Ésik, Z.: Completeness of Park induction. Theor. Comput. Sci. 177(1997) $217-283$

[22] Fischer M. J.: Grammars with macro-like productions. 9th Annual Symposium on Switching and Automata Theory, 1968, 131-142.

[23] Fülöp, Z., Vogler, H.: Tree series transformations that respect copying. Technical Report No. TUD-FI02-01, Technische Universität Dresden, 2002.

[24] Gécseg, F., Steinby, M.: Tree Automata. Akademiai Kiado, 1984.

[25] Gécseg, F., Steinby, M.: Tree Languages. In: Handbook of Formal Languages (Eds.: G. Rozenberg and A. Salomaa), Springer, 1997, Vol. 3, Chapter $1,1-68$.

[26] Ginsburg, S.: Algebraic and Automata-Theoretic Properties of Formal Languages. North-Holland, 1975.

[27] Goguen, J. A., Thatcher, J. W., Wagner, E. G., Wright, J. B.: Initial algebra semantics and continuous algebras. J. Assoc. Comput. Mach. 24(1977) 68-95.

[28] Goldstern, M.: Vervollständigung von Halbringen. Diplomarbeit, Technische Universität Wien, 1985.

[29] Grätzer, G.: Universal Algebra, 2nd ed., Springer, 1979.

[30] Gruska, J.: A characterization of context-free languages. Journal of Computer and System Sciences 5(1971) 353-364.

[31] Guessarian, I.: Algebraic Semantics. Lect. Notes Comput. Sci. 99, Springer, 1981.

[32] Guessarian, I.: Pushdown tree automata. Math. Systems Theory 16(1983) $237-263$.

[33] Karner, G.: On limits in complete semirings. Semigroup Forum 45(1992) $148-165$. 
[34] Krob, D.: Monoides et semi-anneaux continus. Semigroup Forum 37(1988) 59-78.

[35] Kuich, W.: The Kleene and the Parikh theorem in complete semirings. ICALP87, Lect. Notes Comput. Sci. 267(1987) 212-225.

[36] Kuich, W.: Semirings and formal power series: Their relevance to formal languages and automata theory. In: Handbook of Formal Languages (Eds.: G. Rozenberg and A. Salomaa), Springer, 1997, Vol. 1, Chapter 9, 609-677.

[37] Kuich, W.: Gaußian elimination and a characterization of algebraic power series. MFCS 98, LNCS 1450(1998) 512-521.

[38] Kuich, W.: Formal power series over trees. In: Proceedings of the 3rd International Conference Developments in Language Theory (S. Bozapalidis, ed.), Aristotle University of Thessaloniki, 1998, pp. 61-101.

[39] Kuich, W.: Tree transducers and formal tree series. Acta Cybernetica 14(1999) 135-149.

[40] Kuich, W.: Full abstract families of tree series I. In: Jewels are Forever. (J. Karhumäki, H. Maurer, G. Paun, G. Rozenberg, eds.). Springer, 1999, $145-156$.

[41] Kuich, W.: Abstract families of tree series II. In: Proceedings of the International Workshop on Grammar Systems 2000 (R. Freund, A. Kelemenova, eds.), Schlesische Universität Troppau, 2000, pp. 347-358.

[42] Kuich, W.: Formal series over algebras. In: Proceedings of MFCS 2000, LNCS 1893, Springer, 2000, pp. 488-496.

[43] Kuich, W.: Pushdown tree automata, algebraic tree systems, and algebraic tree series. Information and Computation 165(2001) 1-31.

[44] Formal power series over sorted algebras. To appear in Discrete Math.

[45] Kuich, W., Salomaa, A.: Semirings, Automata, Languages. EATCS Monographs on Theoretical Computer Science, Vol. 5. Springer, 1986.

[46] Lausch, H., Nöbauer, W.: Algebra of Polynomials. North-Holland, 1973.

[47] Manes, E. G., Arbib, M. A.: Algebraic Approaches to Program Semantics. Springer, 1986.

[48] Markowsky, G.: Chain-complete posets and directed sets with applications. Algebra Universalis 6(1976) 53-68.

[49] Niwiński, D.: On fixed-point clones (extended abstract). Automata, languages and programming (Rennes, 1986), 464-473, Lecture Notes in Comput. Sci., 226, Springer, Berlin, 1986. 
[50] Rounds, W. C.: Trees, transducers and transformations. PhD thesis, Stanford University, 1968.

[51] Rounds, W. C.: Context-free grammars on trees. ACM Symposium on Theory of Computing, 1969, pp. 143-148. EATCS Monographs on Theoretical Computer Science, Vol. 5. Springer, 1986.

[52] Rounds, W. C.: Mappings and grammars on trees. Math. Systems Theory $4(1970)$ 257-287.

[53] Sakarovitch, J.: Kleene's theorem revisited. Lect. Notes Comput. Sci. 281(1987) 39-50.

[54] Salomaa, A.: Formal Languages. Academic Press, 1973.

[55] Seidl, H.: Deciding equivalence of finite tree automata. STACS88, Lect. Notes Comput. Sci. 349(1989) 480-492.

[56] Thatcher, J. W.: Generalized ${ }^{2}$ sequential machine maps. IBM Research Report RC 2466, 1969.

[57] Thatcher, J. W.: Generalized sequential machine maps. J. Comp. Syst. Sci. 4(1970) 339-367.

[58] Thatcher, J. W., Wright, J. B.: Generalized finite automata theory with an application to a decision problem of second-order logic. Math. Systems Theory 2(1968) 57-81.

[59] Wechler, W.: Universal Algebra for Computer Scientists. EATCS Monographs on Computer Science, Vol. 25. Springer, 1992. 


\section{Recent BRICS Report Series Publications}

RS-02-21 Zoltán Ésik and Werner Kuich. Formal Tree Series. April 2002. $66 \mathrm{pp}$.

RS-02-20 Zoltán Ésik and Kim G. Larsen. Regular Languages Definable by Lindström Quantifiers (Preliminary Version). April 2002. 56 pp.

RS-02-19 Stephen L. Bloom and Zoltán Ésik. An Extension Theorem with an Application to Formal Tree Series. April 2002. 51 pp.

RS-02-18 Gerth Stølting Brodal and Rolf Fagerberg. Cache Oblivious Distribution Sweeping. April 2002. To appear in 29th International Colloquium on Automata, Languages, and Programming, ICALP '02 Proceedings, LNCS, 2002.

RS-02-17 Bolette Ammitzbøll Madsen, Jesper Makholm Nielsen, and Bjarke Skjernaa. On the Number of Maximal Bipartite Subgraphs of a Graph. April 2002. 7 pp.

RS-02-16 Jiří Srba. Strong Bisimilarity of Simple Process Algebras: Complexity Lower Bounds. April 2002. To appear in 29th International Colloquium on Automata, Languages, and Programming, ICALP '02 Proceedings, LNCS, 2002.

RS-02-15 Jesper Makholm Nielsen. On the Number of Maximal Independent Sets in a Graph. April 2002. 10 pp.

RS-02-14 Ulrich Berger and Paulo B. Oliva. Modified Bar Recursion. April 2002. 23 pp.

RS-02-13 Gerth Stølting Brodal, Rune B. Lyngsø, Anna Östlin, and Christian N. S. Pedersen. Solving the String Statistics Problem in Time $O(n \log n)$. March 2002. To appear in 29th International Colloquium on Automata, Languages, and Programming, ICALP '02 Proceedings, LNCS, 2002.

RS-02-12 Olivier Danvy and Mayer Goldberg. There and Back Again. March 2002. This report supersedes the earlier report BRICS RS-01-39.

RS-02-11 Aske Simon Christensen, Anders Møller, and Michael I. Schwartzbach. Extending Java for High-Level Web Service Construction. March 2002. 